\title{
Strategic communication and manipulation
}

Citation for published version (APA):

Aradhye, A. A. (2021). Strategic communication and manipulation. [Doctoral Thesis, Maastricht University]. Maastricht University. https://doi.org/10.26481/dis.20210902aa

Document status and date:

Published: 01/01/2021

DOI:

10.26481/dis.20210902aa

Document Version:

Publisher's PDF, also known as Version of record

\section{Please check the document version of this publication:}

- A submitted manuscript is the version of the article upon submission and before peer-review. There can be important differences between the submitted version and the official published version of record.

People interested in the research are advised to contact the author for the final version of the publication, or visit the DOI to the publisher's website.

- The final author version and the galley proof are versions of the publication after peer review.

- The final published version features the final layout of the paper including the volume, issue and page numbers.

Link to publication

\footnotetext{
General rights rights.

- You may freely distribute the URL identifying the publication in the public portal. please follow below link for the End User Agreement:

www.umlib.nl/taverne-license

Take down policy

If you believe that this document breaches copyright please contact us at:

repository@maastrichtuniversity.nl

providing details and we will investigate your claim.
}

Copyright and moral rights for the publications made accessible in the public portal are retained by the authors and/or other copyright owners and it is a condition of accessing publications that users recognise and abide by the legal requirements associated with these

- Users may download and print one copy of any publication from the public portal for the purpose of private study or research.

- You may not further distribute the material or use it for any profit-making activity or commercial gain

If the publication is distributed under the terms of Article $25 \mathrm{fa}$ of the Dutch Copyright Act, indicated by the "Taverne" license above, 
Doctoral thesis

\section{STRATEGIC COMMUNICATION AND MANIPULATION}

Aditya Aradhye

2021 
(C) Aditya Aradhye, Maastricht 2021.

All rights reserved. No part of this publication may be reproduced, stored in a retrieval system or transmitted in any form or by any means, electronic, mechanical, photocopying, recording or otherwise, without prior written permission of the author.

This book was typeset by the author using $\mathrm{HT}_{\mathrm{E}} \mathrm{X}$.

Cover based on the art from Vecteezy and iStock. Printed in The Czech Republic by Copy General 


\title{
STRATEGIC COMMUNICATION AND MANIPULATION
}

\author{
Dissertation \\ to obtain the degree of Doctor at Maastricht University, \\ on the authority of the Rector Magnificus Prof.dr. Rianne M. Letschert \\ in accordance with the decision of the Board of Deans, \\ to be defended in public \\ on Thursday, September 02, 2021 at 14.00 hours \\ by
}

Aditya Ajit Aradhye 


\section{Supervisors}

Prof. Dr. Dries Vermeulen

\section{Co-supervisors}

Dr. János Flesch

Dr. Mathias Staudigl

\section{Assessment Committee}

Prof. Dr. Stan van Hoesel (Chair)

Prof. Dr. Ehud Lehrer (Tel Aviv University)

Prof. Dr. Jérôme Renault (Toulouse School of Economics)

Dr. Ton Storcken

Dr. Anna Zseleva

This research was financially supported by the Graduate School of Business and Economics (GSBE). 
To my family, for their

unconditional love and eternal support 



\section{Acknowledgments}

I would like start by thanking my supervisors Dries Vermeulen, János Flesch and Mathias Staudigl. Having three supervisors with different research styles and topics of interests helped me a lot to see problems from different perspectives. I enjoyed and learned a lot from the conversations we had about research, which would often turn philosophical at some point. I could see myself growing from someone who hardly understood anything from those discussions to someone who could actively participate and have my own input.

János, you were always very approachable, it was very convenient to drop by your office whenever I needed advice regarding any aspect of the PhD life. Our one on one meetings were very helpful for me to learn the fundamentals like the art of writing the introduction, reviewing the related literature, etc. It was very nice of you to often join us for beers, movies and board games, where we could talk about life apart from work! Dries, the board game nights and dinners at your house were a lot of fun. It was great to see the competitive side of you, when it comes to roboRally! Thank you so much Mathias for the straightforward realistic discussions about the academia, which prepared me for the worst case scenarios. I am very thankful that János and Dries encouraged me to collaborate also with people apart from the supervisors, which led me have an opportunity to work with Hans Peters and Ehud Lehrer.

Finally I would also like to thank Dries, János and Mathias for their patience with me. I can think of many instances where I was too adamant to agree with them on certain issues, only to realize a week later that they were completely right. I didn't always finish all of my responsibilities within time. I am very grateful for their support regardless of everything. 
During my time in Maastricht, I was fortunate enough to have a project with Hans Peters, with whom I could work on one of my passions, social choice theory. Hans, it was real pleasure to work with you. I really enjoyed the conversations during our meetings and couple of Amsterdam visits, and many many interesting stories of so many people that you have encountered.

Spending three months in Tel Aviv with the group of Ehud Lehrer and Eilon Solan was an amazing experience, and it couldn't have been possible without János. Ehud, although I was bit intimidated before meeting you for the first time, your extremely friendly and helpful personality made working with you very pleasant. Our meetings at Aroma were very enjoyable. Eilon, our lunch talks were a lot of fun, I only knew about your research before, but I got to know that you have great management skills as well. Galit, thanks for showing us around in Tel Aviv, we had great time with Steffen, Ilia, Siyu, Anna, János.

I would like to thank the assessment committee for taking the time to carefully read the thesis, Stan van Hoesel, Ehud Lehrer, Jérôme Renault, Ton Storcken, Anna Zseleva, and would like to thank Arunava Sen and Bas Dietzenbacher for agreeing to be part of my defense committee. I would also like to thank Karin, Yolanda and Vera for being the most efficient and friendly secretaries. I would like to thanks Shash and Benoit for agreeing to be my paranymphs.

Shash, you have been a guide for me about everything I needed to know about Dutch life for an Indian, even before I decided to come to Maastricht. I was very happy to be your office mate officially for an year, and then unofficially for 2 more years. Thanks for letting me use your couch and your bike more times than I can recall. It was nice to have a friend who shares the same level of enthusiasm about comic books and superhero movies. Thanks for introducing me to the wonder of specialty dark beers. Benoit, it was great to have a friend with so many hobbies in common, particularly that of board games and math puzzles. It was great to attend countless number of conferences and seminars together, and to discuss the topics afterwards. It was very 
nice to visit Biarritz two times, first time especially was very special as I could experience an authentic family Christmas. Thank you for making me more aware about the environment. Thanks Luca for agreeing with me that La Chouffe at Tribunal tastes much better than any other place, maybe it had something to do with the peanuts. I learnt the hard way from you that chicken in pasta is as bad as pineapple on pizza, although I am happy that in the end I managed to make 'acceptable' Fettuccine Alfredo. Thank you for sharing your knowledge of Scotch whiskeys and Italian liquors. Our trip with Benoit and Niels to Milan and Tuscany was amazing! Thanks Niels for helping me to understand the Dutch culture, and for being our official Dutch to English translator. You and Benoit helped me a lot with the formalities of the PhD defense, many thanks for that. Your knowledge of historical facts and random trivia helped us in pub quizzes, and made the conversations much more interesting. I can't say that for other people, but I loved your unique sense of humour!

Farzaneh, it was very interesting to see you integrating slowly in the European culture, it reminded me of my early days here. Among the three neighbours you had, I was clearly the best one, you are welcome! Thanks for teaching me some delicious Iranian dishes. I still wonder how we didn't kill each other, after being stuck during the lockdown. Niloofar, I don't know how we always end up having crazy time, like almost dying on roller coaster, playing consecutive 30 games of Monopoly deal. It was either that, or having talks about the meaning of life and happiness. There was no middle ground. I miss how we always spent time joking around and pulling each other's legs. Anna, although we were colleagues at Maastricht only for a short time, it was real pleasure to know you from so many conferences and during my time in Tel Aviv. I got so many useful suggestions from you about $\mathrm{PhD}$ life, from colleague interaction to job market. I really enjoyed our extensive conversations about different styles of TV shows. Aida, the ginger dinners were such a delight. I am so glad that we could visit Jerusalem and Dead Sea, wish we can go there again. I am sure that I will see you again at some random conference. 
Caterina, few mountain biking trips we had were a lot of fun, in spite of the flat tires! Thanks for teaching me statistics enough to teach QM course. Li, it was always nice to have an insider to tell us about the specials at ginger before they got sold out. Sumon, I was glad to have you as my office-mate for two years. Our coffee breaks were nice little distraction from work. Dewi, I really enjoyed our coffee breaks during the teaching, arguably my favourite part of the teaching. Roland, I really enjoyed our talks about language families, memes, and mutual weird sense of humour. Thanks Ilo for getting us started with Bridge, DnD, wish that you could have joined us more after! Aiste, It was fun to explore parts of Brussels with Benoit. Ilaria, I enjoyed our small coffee meetups, thank you for all the Swiss chocolates. Tim, Marc, Veerle, Sean, Hanno, Alex, it was great to hear interesting past stories which helped me to integrate into KE culture. I would also like to thank Bas, Qian, Moritz, Yicong, Elisa, Son, Julian, Rasmus for being cool colleagues.

Coming from India, many aspects of life in Maastricht were quite new and often confusing. Having a close group of Indian friends made this transition much easier. The important thing that we all missed was Indian food. So, it was great to have weekly meetups with Jaspreet, Raghav, Ujjwala, Shash, Atul, Stuti, Terina, Chinmay, Vineet to cook together and talk about what we all missed from back home. Jaspreet and Raghav, it was great to watch and analyze so many movies, using our Pathe unlimited card. Atul, it was great to travel with you for two weeks and experience Christmas in different cities, I hope we can travel together again soon. Ujj, it was nice to explore the Randstad, even though it took us more than two years to finally visit some of these places. Vineet the most enthusiastic among us, you throw amazing Diwali, Dussehra parties, the snooker games with Atul and Shash were really enjoyable. Terina, Stuti, Chinmay, it was nice to have coffee together again after the first lockdown.

Finally I would like to my thank my support system, my family. Thank you Aai, Baba, and Aboli Tai. Thank you so much for all of your love, support and care. Our regular talks were very relaxing whenever I felt 
tensed. Baba, you might hardly understand any details of my work, but your guidance and suggestions helped me a lot to plan everything. Aai, all the sweets and snacks you sent were such a treat after eating Dutch food. Thanks for designing the cover page of this thesis for me. Tai, all of your movie and music suggestions were a nice distraction from work. Ajji, thank you for constant support. Amit dada, it was very nice to compare with you the life in academics with the life in industry. And finally little Dhrupad, it was a real joy to watch you grow up so fast, you made last year of my PhD much brighter. I am grateful to all of you!

Aditya Aradhye 



\section{Contents}

Acknowledgments vii

1 Introduction 1

2 Incentive compatibility in sender-receiver stopping games 7

2.1 Introduction .............. 8

2.1.1 Our contribution ................ 9

2.1.2 Related literature .............. 11

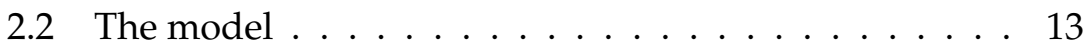

2.2.1 The game .................. 13

2.2.2 Strategies and expected payoffs . . . . . . . . . 14

2.2.3 Perfect Bayesian equilibrium . . . . . . . . . . 15

2.2.4 Terminology for strategies . . . . . . . . . 16

2.3 The regular strategy profile . . . . . . . . . . 17

2.3.1 Finite horizon ............. 18

2.3.2 Infinite horizon ............. 20

2.4 Existence and unicity of PBE, finite horizon . . . . . . . 21

2.5 Existence and unicity of PBE, infinite horizon . . . . 23

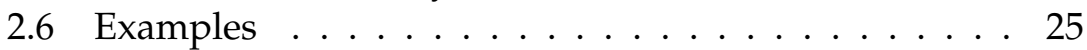

2.7 Concluding remarks . . . . . . . . . . . . . . . . 27

2.8 The proofs .................. 28

2.8.1 The proof of Theorem 2.5 . . . . . . . . . . 28

2.8.2 The proof of Theorem 2.6 . . . . . . . . . 36

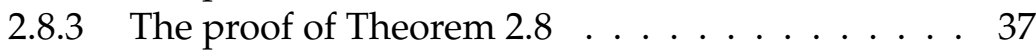

2.8.4 The proof of Theorem 2.9 . . . . . . . . 43

3 Sender-Receiver stopping games with multiple senders 57

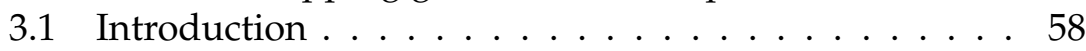

3.1.1 Our contribution ............. 59

3.1.2 Related literature ............ 61 
3.2 The model . . . . . . . . . . . . . . . . . . 62

3.2 .1 The game ............... . . 63

3.2 .2 Strategies . . . . . . . . . . . . . . 64

3.2.3 Perfect Bayesian equilibrium . . . . . . . . . . . 64

3.2.4 Terminology for strategies . . . . . . . . . . . . 66

3.3 Regular strategy profile . . . . . . . . . . . . . . . 67

3.3.1 Sincere strategy of the senders . . . . . . . . . . 67

3.3.2 Reactive strategy of the receiver . . . . . . . 68

3.3.3 Regular strategy profile . . . . . . . . . . . . 69

3.4 Main results . . . . . . . . . . . . . . . . . . . . . . 71

3.4 .1 Preparations . . . . . . . . . . . . . 71

3.4 .2 Results . . . . . . . . . . . . . . . 73

3.5 Illustrations and examples . . . . . . . . . . . . . 84

3.5.1 An example: 2 senders . . . . . . . . . . . . . . . . 84

3.5 .2 Other PBEs . . . . . . . . . . . . . . . . 87

3.6 Externality of additional senders . . . . . . . . . . 88

3.6.1 Externality of additional sender for the senders . 89

3.6.2 Externality of additional senders for the receiver . 90

3.7 Concluding remarks . . . . . . . . . . . . 92

4 Group strategy-proof rules in multidimensional binary domains 99

4.1 Introduction . . . . . . . . . . . . . . . . . . 100

4.2 Model and preliminary results . . . . . . . . . . . 103

4.2.1 Model and definitions . . . . . . . . . . . . . 103

4.2.2 Further notations . . . . . . . . . . . . . . . 105

4.2 .3 Preliminary results . . . . . . . . . . . . 106

4.3 Strategy-proofness . . . . . . . . . . . . . . . 106

4.3.1 Switching points . . . . . . . . . . . . 107

4.3.2 Component-wise majority rules . . . . . . . . 108

4.3.3 Two agents and two components . . . . . . . . . 110

4.3.4 Further results on strategy-proofness . . . . . . 111

4.4 Weak group strategy-proofness . . . . . . . . . . . 113

4.5 Strong group strategy-proofness . . . . . . . . . . . 117

4.6 Concluding remarks . . . . . . . . . . . . . 122 
Bibliography

Summary

Impact of the Thesis

About the author 



\section{1 \\ Introduction}

Game theory is the study of the strategic interaction taking place between the players with personal motives. The players can represent individuals, firms, animals, etc. and are usually assumed to be selfinterested and rational. The motives take various forms such as money, time, resources, happiness, etc. Game theory has many applications in economics, computer science, political science, biology, philosophy and psychology.

This thesis is divided into two parts which study two different topics in game theory. The first part contains Chapters 2 and 3, which deal with sender-receiver games, and second part contains Chapter 4, which deals with social choice theory.

In dynamic games, players interact repeatedly over a specified duration. Stopping games are dynamic games in which the actions of the players have an effect on whether the game stops at the current period or continues to the next period.

Games of incomplete information are the games where one or more players are uninformed or partially informed about certain parame- 
ters of the game, which may affect their payoffs. These parameters are often drawn from probability distributions, and some of the players are informed only about the distributions, but not the realizations.

One of the well-studied settings in games with incomplete information is sender-receiver games. First introduced by Crawford and Sobel [9], sender-receiver games model the interaction between the fully informed sender and the partially informed receiver. In these games, the actions of the receiver have an influence on the payoff of the sender, and hence the information transmission has a strategic aspect. Dynamic sender-receiver games are the games in which the sender and the receiver interact repeatedly for a fixed duration.

In Chapter 2, we introduce a model of sender-receiver stopping games, where the state of the world follows an iid-process throughout the game. At each period, the sender observes the current state, and sends a message to the receiver, suggesting either to stop or to continue. The receiver, only seeing the message but not the state, decides either to stop the game, or to continue which takes the game to the next period. The payoff to each player is a function of the state when the receiver quits. The horizon of the game can be finite or infinite.

We assume that rewards are positively correlated with the state of nature, i.e. higher states lead to better payoffs for both players. Thus, both player have identical ordinal preferences over realizations of the state of nature. Yet, as we impose no further restrictions on the payoffs, the cardinal assignment of values of the two payoff functions can be very different. As a consequence, a certain state may be very appealing to one player, but not so much to the other, creating an interesting strategic tension between the parties. We investigate to what extent the cardinal differences may hamper coordination between the players.

We prove existence and uniqueness of non-babbling Perfect Bayesian Equilibrium (PBE) under mild conditions on the game primitives in the case where the players are sufficiently patient. This PBE has a remarkably simple structure, which builds on the identification of an easy-to-implement and compute class of threshold strategies for the 
sender. With the help of these threshold strategies, we derive simple expressions describing this PBE.

It turns out that in this PBE the receiver obediently follows the recommendations of the sender. Hence, the sender alone plays the decisive role, and regardless of the payoff function of the receiver the sender always obtains the best possible payoff for himself. One of the natural questions is to ask: How do these results change, when we have multiple senders?

In Chapter 3, we consider the model of sender-receiver stopping games with multiple senders. The state of the world follows an iid-process throughout the game. At each period, each sender observes the current state and sends a message to the receiver, suggesting either to stop or to continue. The receiver, only seeing the collections of messages but not the state, decides either to stop the game, or to continue which takes the game to the next period. The payoff to each player is a function of the state when the receiver quits, with higher states leading to better payoffs. The horizon of the game is finite.

We prove the existence of a non-babbling (PBE) where the players are sufficiently patient. In this PBE the senders play threshold strategies which can be computed recursively. The receiver's strategy is constructed with the help of these threshold strategies. According to this strategy, the receiver follows the recommendations of different senders at different periods. This strategy has remarkably simple structure; it is a threshold strategy where the choice of the thresholds has nice intuitive interpretation. This PBE is also sender coalition-proof, that is, there does not exist a deviation by a coalition of senders which is strictly beneficial to each sender in the coalition.

Having more senders means more information for the receiver. One can think that the receiver should be (at least weakly) better off in the game with more senders. However it tuns out that, with the above PBE as a focal equilibrium, there are games in which the receiver is strictly worse off if with more senders. Hence, the receiver is not always able to exploit the additional information. 
Social choice theory is the study of collective decision making. The central question is: Can we design a rule for choosing a winning outcome from a given set of options, in which agents do not have any incentive to misreport their preferences? Gibbard [16] and Satterthwaite [33] show that if there are at least 3 agents, the only unanimous social choice function which provides incentives for each agent to report his private information truthfully is one where the responsibility of choosing the outcome is left solely to a single dictator. This result is not necessary true, however, when the preference domain is restricted. In Chapter 4, we study one such domain.

In Chapter 4, we consider a setting in which the alternatives of the agents are binary vectors and the preferences of the agents are determined by the Hamming distance from their most preferred alternative. We restrict our attention to unanimous, anonymous, and neutral social choice functions, and focus on strategy-proofness, weak group strategy-proofness, and strong group strategy-proofness. Our results range from possibility results concerning strategy-proofness and weak group strategy-proofness, to impossibility of strong group strategyproof social choice functions.

In our setting, it turns out that strategy-proofness is a fairly weak requirement. We show that component-wise majority rules are strategyproof. A component-wise majority rule simply assigns to each component a zero if the majority of agents assigns a zero to that component, and a one if the majority of agents assigns a one, with a tie-breaker in the case that the number of agents is even. Even for the simple case of two agents and two components, describing all strategy-proof rules is a rather elaborate task, and we learn from it that, without additional conditions, characterizing all strategy-proof rules for the general case will be practically infeasible. Hence notions stronger than strategyproofness should be considered.

In weak group strategy-proof rules, there is no coalition of agents in which each agent is strictly better off, and in strong group strategy-proof rules, there is no coalition of agents in which each 
agent is weakly better off and at least one agent is strictly better off. Somewhat surprisingly, it turns out that for at least four agents and at least three components, component-wise majority rules are not weakly group strategy-proof. This, however, does not mean that there are no weakly group strategy-proof rules. Then we show that for at least four agents and at least three components, there exists no strong group strategy-proof rule. 



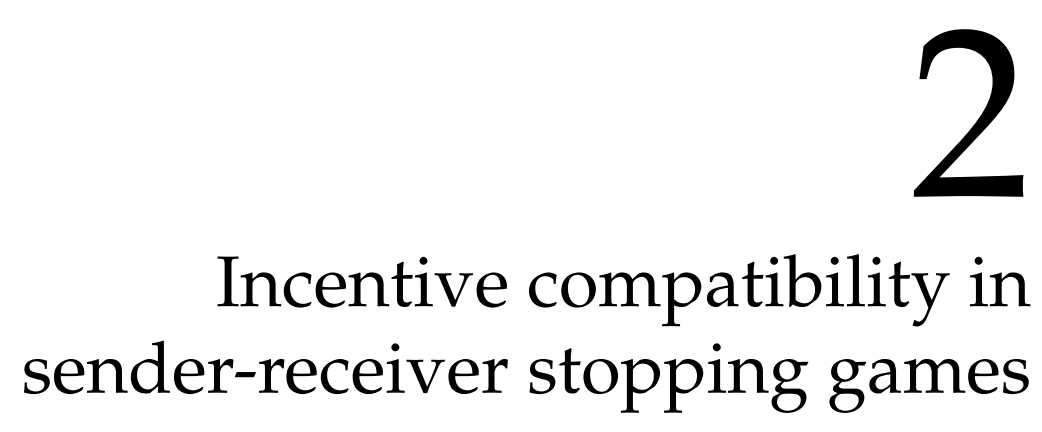

Adapted from: Aditya Aradhye et al. "Incentive compatibility in sender-receiver stopping games". In: arXiv preprint arXiv:2004.01910 (2020). 


\subsection{Introduction}

Information transmission is a fundamental element of economic models. In various settings, a better informed party (sender) is in the position to transmit information to a lesser informed or even uninformed party (receiver). Typically, the action choices of the receiver have an influence on the payoff of the sender, and hence the information transmission has a strategic aspect. In their seminal paper, Crawford and Sobel [9], analyze strategic information transmission with a single interaction between the sender and the receiver. Their model and its variations $[9,18]$ have a wide range of applications, notably in economics, computer science, political science but also in biology and philosophy $[4,35,20]$. Recently, a few models have been introduced in which the information transmission takes place in a dynamic setting, see for instance [31] and [17]. In these models, the sender-receiver game is played repeatedly either on a finite or an infinite horizon and the payoff for the sender and the receiver is the total discounted sum of the stage payoffs. The key focus in these papers is the characterization of the set of equilibrium payoffs where, in the spirit of the folk theorem, the players are sufficiently patient.

This chapter ${ }^{1}$ sets the stage for a different line of research, which we regard as an important conceptual contribution of our work. Specifically, this chapter introduces a model of sender-receiver stopping games, which combines features from dynamic sender-receiver games and stopping games (for a survey on the latter, see [37]). In these games the strategic information transmission takes place repeatedly until the receiver decides to stop the interaction. More precisely, we deal in this chapter with sender-receiver stopping games of finite as well as infinite horizon. In the finite horizon, the receiver is forced to stop the game before a pre-defined terminal period has been reached. In the infinite horizon the game may be played for unlimited number of periods. The tim-

\footnotetext{
${ }^{1}$ This chapter is based on joint work with János Flesch, Mathias Staudigl and Dries Vermeulen. I would like to thank Galit Ashkenazi-Golan, Gaëtan Fournier, Jérôme Renault, Eilon Solan and Bruno Ziliotto for helpful discussions.
} 
ing of the game is as follows: In each period nature draws a state of the world, which is only revealed to the sender. After observing the state of the world, the sender sends one out of two messages to the receiver. This message is interpreted as a suggestion either to stop the game or to continue. Now the receiver has to take a decision. After seeing the message, but without knowing the state, the receiver has two options: he can decide to stop the game, or he can decide to continue to the next period. The payoff to each player is a function of the state at which the receiver stops, and these payoffs are either discounted or undiscounted. The setting could be thought as an investor (the receiver) who must make an irreversible financial decision, without having exact information about the market situation, but using the advice of an expert (the sender).

We assume that rewards are positively correlated with the state of nature, i.e. higher states lead to better payoffs for both players. Thus, both player have identical ordinal preferences over realizations of the state of nature. Yet, as we impose no further restrictions on the payoffs, the cardinal assignment of values of the two payoff functions can be very different. As a consequence, a certain state may be very appealing to one player, but not so much to the other, creating an interesting strategic tension between the parties. This chapter investigates to what extent the cardinal differences may hamper coordination between the players.

The main solution concept that we use to analyze these games is Perfect Bayesian Equilibrium (PBE), and we identify a class of PBEs which are appealing to the players in terms of payoffs, and moreover easy to compute and implement.

\subsubsection{Our contribution}

We are interested in PBEs in which the receiver plays a responsive strategy. We call a receiver's strategy responsive if his mixed stage game action is different for different messages sent by the sender. This 
is just a condition which excludes those PBEs in which the receiver's strategy is babbling, which are fairly uninteresting from a strategic perspective. $^{2}$

Therefore, the crucial question is how the sender should use his additional information to manipulate the choices of the receiver given the current state, and to what extent the receiver can trust the sender's recommendations and be obedient. In particular, we investigate under what conditions the incentives of the sender and the receiver match.

These concerns are captured by the notion of a regular strategy profile. In a regular strategy profile, the receiver simply follows the sender's recommendations, whereas the sender sends a sincere message, given the realization of the state, whether or not he would like the receiver to terminate the game at this period. Since the receiver is obedient, he is not playing an active role in such a strategy profile. The sender's sincere strategy is a threshold strategy that sends the message "continue" if the current state is below the threshold and sends the message "quit" otherwise. In other words, the sender's strategy is the optimal solution of the one player maximization problem in which the decision to continue or to quit is delegated to the sender. This means that in the regular strategy profile the sender obtains the best possible payoff for himself. Indeed, this outcome is Pareto optimal. The game we study admits an (essentially) unique ${ }^{3}$ regular strategy profile.

In finite horizon, we show that there is no responsive PBE other than the regular strategy profile. Even the regular strategy profile may fail to exist in certain games if the discount factor is small. More precisely, our findings are as follows:

(i) The regular strategy profile is the unique responsive PBE if the

\footnotetext{
${ }^{2}$ Regardless, it is fairly easy to study all the PBEs which are not responsive by simply adding babbling periods in any responsive PBE.

${ }^{3} \mathrm{By}$ (essentially) unique, we mean that in any two regular strategy profiles, the actions of the players differ at only measure zero sets and thus induce the same expected payoffs for the players.
} 
discount factor is sufficiently high or if the payoffs are undiscounted.

(ii) There are games that have no responsive PBE if the discount factor is small enough.

For the infinite horizon, we focus on strategy profiles where the expected payoffs after any period do not depend on the history. We show that within this class of strategy profiles, the regular strategy profile is the unique responsive PBE provided the discount factor is sufficiently high. More precisely, our findings are as follows:

(iii) The regular strategy profile is the unique responsive PBE if the discount factor is sufficiently high. In this setting, the regular strategy profile is stationary.

(iv) There are games that have no responsive PBE if the discount factor is small enough.

In the extreme case of infinite horizon where the payoffs are undiscounted, there does not exist any responsive PBE. This is due to the fact that the sender does not have a best response when the receiver is obeying, and hence, it should not be interpreted as a breakdown of communication.

\subsubsection{Related literature}

Crawford and Sobel [9] introduced a model of strategic information transmission. The model in which the sender and the receiver interact only once is studied extensively, see $[9,18]$. Recently a lot of work has been focused on the dynamic extension, where the strategic interaction takes place repeatedly either for finite or infinite number of periods, see $[31,17,25,3]$. [31] assume that the sequence of states follows an irreducible Markov chain. They characterize the limit set of equilibrium payoffs, as players become very patient. [17] study finite horizon 
games, and show that, under certain conditions, full information revelation is possible and conditioning future information release on past actions improves incentives for information revelation.

This chapter relates to the large and growing literature of Bayesian persuasion, see $[21,12,32,19]$. In these settings, the informed advisor (i.e. the sender) decides how much information to share with a less informed agent (i.e. the receiver) so as to influence his decision. [32] show that in many cases, the optimal greedy disclosure policy for the sender exists, which at each stage, minimizes the amount of information being disclosed in that stage under the constraint that it maximizes the current payoff of the sender.

This chapter also relates to the classical contributions on communication in games (see e.g. [14, 29]). In [36, 6], the evolution of information flow is studied in the setting of strategic communication. Our model is also linked to topics in computer science such as automated advice provision. For instance, [4] use sender-receiver games to model interaction between computers and humans.

Most of the previous work has focused on the sender-receiver games with fixed duration of time. Compared to these earlier papers, one of the main novelties of our model is that the game is a stopping game. The receiver has the license to stop the game at any period of time. Some of the techniques we use (for example, backward induction) are also similar to the ones in the literature on stopping games, see [37, 11].

In the responsive $\mathrm{PBE}$, the receiver complies with the sender and the sender tries to maximize his expected payoff without knowing the future states. Hence, our setting in a wider sense, is a variant of the secretary problem, see [13].

The structure of the chapter is as follows. In section 2.2 we introduce the model and in the section 2.3, we discuss the regular strategy profile. In sections 2.4 and 2.5 we state our main results for the finite horizon and the infinite horizon respectively. In section 2.6, we give illustrative 
examples and in section 2.7, we have concluding remarks. In section 2.8 , we provide the proofs of the main theorems.

\subsection{The model}

In this section we describe the model of sender-receiver stopping games and the solution concept of Perfect Bayesian Equilibrium.

\subsubsection{The game}

We study sender-receiver stopping games. These are dynamic games played by two players, the sender and the receiver, either with finite or infinite horizon.

An infinite horizon sender-receiver stopping game is played at periods in $\mathbb{N}=\{1,2, \ldots\}$. At period $t \in \mathbb{N}$, play is as follows. First, a state of the world $\theta^{t}$ is drawn uniformly from the unit interval $I=[0,1]$, independently of the earlier realizations $\theta^{1}, \ldots, \theta^{t-1}$. The sender learns $\theta^{t}$, while the receiver only knows the distribution of $\theta^{t}$. Next, the sender chooses a message $m^{t} \in\left\{m_{c}, m_{q}\right\}$ and sends it to the receiver. The message $m^{t}=m_{c}$ is interpreted as a suggestion for the receiver to continue at this period $t$ and the message $m^{t}=m_{q}$ as a suggestion to quit. On seeing the message, the receiver chooses an action $a^{t} \in\left\{a_{c}, a_{q}\right\}$, where $a_{c}$ stands for continue and $a_{q}$ stands for quit. If the receiver quits then the game ends at period $t$, whereas if the receiver continues then the game proceeds to period $t+1$. If the game ends at period $t$, then the sender receives the payoff $f^{t}\left(\theta^{t}\right)$ and the receiver receives the payoff $g^{t}\left(\theta^{t}\right)$. Here, $f^{t}$ and $g^{t}$ are two continuous and strictly increasing functions from $I$ to $\mathbb{R}_{+}$. If the receiver never quits, both players receive payoff zero, with $f^{t}(0)=g^{t}(0)=0$. For each player, the outcome in which the receiver continues forever is worst. The payoff if the receiver continues forever is zero for each player.

If there are functions $f$ and $g$ such that $f^{t}=f$ and $g^{t}=g$ for all periods $t \in \mathbb{N}$, we say that the game has period-independent payoffs. If there 
are functions $f$ and $g$ and $\delta \in(0,1)$ such that $f^{t}=\delta^{t-1} \cdot f$ and $g^{t}=$ $\delta^{t-1} \cdot g$ for all periods $t \in \mathbb{N}$, we say that the game has discounted payoffs with discount factor $\delta$. In such cases, the functions $f$ and $g$ are called characteristic functions of the game. We assume throughout the chapter that payoffs are either period-independent or discounted.

The model description for sender-receiver stopping games on a finite horizon $T \in \mathbb{N}$ is almost identical to the one above. The only modification is that if period $T$ is reached then the game terminates at the end of period $T$. Now we proceed with the description of sender-receiver stopping games on the infinite horizon, without explicitly mentioning the changes for the finite horizon.

\subsubsection{Strategies and expected payoffs}

Histories. For the sender, a history at period $t$ is a sequence $h_{s}^{t}=$ $\left(\theta^{1}, m^{1}, \ldots, \theta^{t-1}, m^{t-1}\right)$ of past states and messages sent by the sender. By $H_{s}^{t}=(I \times M)^{t-1}$ we denote the set of histories for the sender at period $t$. Given the usual topology on $I$, we endow $H_{s}^{t}$ with the product Borel sigma-algebra.

Since the receiver does not observe the realization of the states, a history of the receiver at period $t$ is a sequence $h_{r}^{t}=\left(m^{1}, \ldots, m^{t-1}\right)$ of past messages sent by the sender. By $H_{r}^{t}=M^{t-1}$ we denote the set of histories for the receiver at period $t$. Note that $H_{r}^{t}$ is a finite set.

Strategies. A strategy $\sigma=\left(\sigma^{t}\right)_{t=1}^{\infty}$ for the sender is a sequence of measurable functions $\sigma^{t}: H_{s}^{t} \times I \rightarrow[0,1]$. The interpretation is that, at each period $t$, given the history $h_{s}^{t}$ and the state $\theta^{t}$, the strategy $\sigma^{t}$ places probability $\sigma^{t}\left(h_{s}^{t}, \theta^{t}\right)$ on the message $m_{c}$.

A strategy $\tau=\left(\tau^{t}\right)_{t=1}^{\infty}$ for the receiver is a sequence of functions $\tau^{t}: H_{r}^{t} \times M \rightarrow[0,1]$. We do not need any measurability conditions for $\tau^{t}$ as the domain of $\tau^{t}$ is finite. The interpretation is that, at each period $t$, given the history $h_{r}^{t}$ and the message $m^{t}$, the strategy $\tau^{t}$ places probability $\tau^{t}\left(h_{r}^{t}, m^{t}\right)$ on the action $a_{c}$. 
For the case when the game has a finite horizon $T$, for simplicity we require that at period $T$, regardless the history, the sender's strategy has to send the message $m_{q}$ and the receiver's strategy has to play the action $a_{q}$.

In this model, we focus on the responsive strategies of the receiver. A strategy $\tau$ of the receiver is called responsive if, for each period $t$ (with $t<T$ if the game has finite horizon $T$ ) and history $h_{r}^{t}$, we have $\tau^{t}\left(h_{r}^{t}, m_{c}\right)>\tau^{t}\left(h_{r}^{t}, m_{q}\right)$. This is saying that, upon receiving the message $m_{c}$, the receiver chooses action $a_{c}$ with higher probability than upon receiving $m_{q}{ }^{4}$

\subsubsection{Perfect Bayesian equilibrium}

In this section we introduce the solution concept we use to analyse the sender-receiver stopping games defined above.

Consider a strategy profile $(\sigma, \tau)$. The expected payoffs of the sender and the receiver are denoted by $U_{s}(\sigma, \tau)$ and $U_{r}(\sigma, \tau)$ respectively. If the receiver has not quit until some period $t$, and the histories are $h_{s}^{t}$ and $h_{r}^{t}$ respectively, then the continuation expected payoffs from period $t$ onward are denoted by $U_{s}^{t}(\sigma, \tau)\left(h_{s}^{t}\right)$ and $U_{r}^{t}(\sigma, \tau)\left(h_{r}^{t}\right)$ respectively. For details on the definitions of these notations, refer to the Appendix B.

We say that, at period $t$, the expected payoff $U_{s}^{t}(\sigma, \tau)$ for the sender is history independent if for every $h_{s}^{t}, \bar{h}_{s}^{t} \in H_{s}^{t}$ it holds that $U_{s}^{t}(\sigma, \tau)\left(h_{s}^{t}\right)=$ $U_{s}^{t}(\sigma, \tau)\left(\bar{h}_{s}^{t}\right)$. Note that history independence of $U_{s}^{t}(\sigma, \tau)$ is equivalent to saying that the function $U_{s}^{t}(\sigma, \tau)$ is constant. In that case, with slight abuse of notation, we identify the function with the (constant) value of that function, and act as if $U_{s}^{t}(\sigma, \tau)$ is a real number instead of a function. A similar observation holds for the expected payoff $U_{r}^{t}(\sigma, \tau)$ for the receiver.

\footnotetext{
${ }^{4}$ The reason to restrict our attention to responsive strategies is to avoid PBEs in 'babbling' strategies, which are fairly uninteresting from a game theory perspective.
} 
Chapter 2. Incentive compatibility in sender-receiver stopping games

Definition 2.1. A strategy profile $(\sigma, \tau)$ is called a Perfect Bayesian Equilibrium (PBE) if for every period $t$, and every history $h_{s}^{t}$, we have $U_{s}^{t}(\sigma, \tau)\left(h_{s}^{t}\right) \geq U_{s}^{t}\left(\sigma^{\prime}, \tau\right)\left(h_{s}^{t}\right)$ for every strategy $\sigma^{\prime}$ of the sender, and for every $h_{r}^{t}, U_{r}^{t}(\sigma, \tau)\left(h_{r}^{t}\right) \geq U_{r}^{t}\left(\sigma, \tau^{\prime}\right)\left(h_{r}^{t}\right)$ for every strategy $\tau^{\prime}$ of the receiver. $A P B E$ is called responsive if the receiver's strategy is responsive. ${ }^{5}$

Notice that in the definition of PBE we do not explicitly talk about beliefs of the players on the realized history consisting of the past states, messages and actions. Since the sender's history contains all this information, he is fully informed and he knows the history of the receiver. On the other hand, the receiver is not informed of the past or current states. Based on his own history and the strategy profile $(\sigma, \tau)$, he has a natural belief on the possible histories of the sender, which is compatible with Bayesian updating. For details, we refer to Appendix A.

We will regularly make use of the fact that the well-known one-shot deviation principle holds in our games whenever the game has a finite horizon (regardless whether the payoffs are period-independent or discounted) or the game has infinite horizon and the payoffs are discounted. More precisely, in these settings, a strategy profile $(\sigma, \tau)$ is a PBE if and only if (1) for every history $h_{s}^{t}$ of the sender, we have $U_{s}^{t}(\sigma, \tau)\left(h_{s}^{t}\right) \geq U_{s}^{t}\left(\sigma^{\prime}, \tau\right)\left(h_{s}^{t}\right)$ for every $\sigma^{\prime}$ that is a one-shot deviation from $\sigma$ at $h_{s}^{t}$, and (2) similarly for the receiver. Here, for two strategies $\sigma$ and $\sigma^{\prime}$ and a history $h_{s}^{t}$ of the sender, $\sigma^{\prime}$ is called a one-shot deviation from $\sigma$ at history $h_{s}^{t}$ if $\sigma^{\prime}(h)=\sigma(h)$ for every history $h \neq h_{s}^{t}$ of the sender. One-shot deviations are defined similarly for the receiver.

\subsubsection{Terminology for strategies}

A strategy $\sigma$ for the sender is called pure if, for each period $t$, history $h_{s}^{t}$ and state $\theta^{t}$, either $\sigma^{t}\left(h_{s}^{t}, \theta^{t}\right)=1$ or $\sigma^{t}\left(h_{s}^{t}, \theta^{t}\right)=0$. Pure strategies for the receiver are defined in a similar fashion.

\footnotetext{
${ }^{5} \mathrm{PBE}$ is a refinement of Bayesian Nash Equilibrium (BNE). Intuitively, it requires that the strategy profile induces a BNE after any history.
} 
A strategy $\sigma$ for the sender is said to have a threshold at period $t$, if there exists a number $\beta^{t} \in[0,1]$ such that

$$
\sigma^{t}\left(\theta^{t}\right)= \begin{cases}1 & \text { if } \theta^{t} \in\left[0, \beta^{t}\right) \\ 0 & \text { if } \theta^{t} \in\left(\beta^{t}, 1\right] .\end{cases}
$$

We do not specify what the strategy recommends when the state is exactly equal to the threshold, for the sake of flexible exposition of our results. In any case, this occurs with probability zero only. A strategy $\sigma$ for the sender is called a threshold strategy if it has a threshold at each period $t$. A threshold strategy $\sigma$ is called stationary if $\beta^{s}=\beta^{t}$ for all periods $s$ and $t$.

A strategy profile $(\sigma, \tau)$ is called essentially Markov if $U_{s}^{t}(\sigma, \tau)$ and $U_{r}^{t}(\sigma, \tau)$ are history independent. So the history at period $t$ does not influence the continuation payoffs from period $t$ onward, although it still may influence the continuation strategies.

\subsection{The regular strategy profile}

The regular strategy profile plays a central role in this chapter. A strategy profile $(\sigma, \tau)$ is called regular if $\tau$ is the obedient strategy, and $\sigma$ is sincere against $\tau$. The obedient strategy $\tau$ for the receiver is defined, for each period $t$ and each history $h_{r}^{t}$, by $\tau^{t}\left(h_{r}^{t}, m_{c}\right)=1$ and $\tau^{t}\left(h_{r}^{t}, m_{q}\right)=0$. The obedient strategy is pure, and responsive.

For a given strategy $\tau$ of the receiver, a threshold strategy $\sigma$ of the sender is called sincere against $\tau$ at period $t$ (with $t<T$ if the game has finite horizon $T$ ) if

[1] $U_{s}^{t+1}(\sigma, \tau)$ is history independent, and

[2] the strategy $\sigma$ has the threshold $\alpha^{t}$ at period $t$ where $\alpha^{t}$ is the solution to the equation $f^{t}\left(\alpha^{t}\right)=U_{s}^{t+1}(\sigma, \tau)$. 
Chapter 2. Incentive compatibility in sender-receiver stopping games

A threshold strategy $\sigma$ is called sincere against $\tau$ if it is sincere against $\tau$ at each period $t$ (with $t<T$ if the game has finite horizon $T$ ).

Notice that indeed the equation in condition [2] has a unique solution due to monotonicity of $f^{t}$, and the fact that, since payoffs are either period-independent or discounted, $U_{s}^{t+1}(\sigma, \tau) \leq f^{t}(1)$.

In a regular strategy profile, the sender sends a sincere message whether or not he would like the receiver to terminate the game at this period. Next paragraph provides intuitive explanation on why the condition [2] achieves this.

Assume that $(\sigma, \tau)$ is a regular strategy profile such that $\sigma$ has threshold $\alpha^{t}$ at period $t$. If $\theta^{t}<\alpha^{t}$, then $f^{t}\left(\theta^{t}\right)<f^{t}\left(\alpha^{t}\right)=U_{s}^{t+1}(\sigma, \tau)$. In this case the sender would like the receiver to continue the game as the expected continuation payoff is higher than the expected payoff if the receiver quits. Indeed, the strategy $\sigma$ recommends the message $m_{c}$ as $\theta^{t}<\alpha^{t}$ and $\alpha^{t}$ is the threshold. Similarly, if $\theta^{t}>\alpha^{t}$, then $f^{t}\left(\theta^{t}\right)>f^{t}\left(\alpha^{t}\right)=U_{s}^{t+1}(\sigma, \tau)$ and $\sigma$ recommends the message $m_{q}$.

We argue in both the finite and the infinite horizon model that the regular strategy profile is (essentially) unique, and entirely computable. To explain this, we define the auxiliary function $H: I \rightarrow I$ by

$$
H(x)=f^{-1}\left(\delta \cdot\left[x \cdot f(x)+\int_{x}^{1} f(\theta) d \theta\right]\right) .
$$

\subsubsection{Finite horizon}

Assume that the game has finite horizon $T$. Define the numbers $\beta^{1}, \ldots, \beta^{T}$ as follows ${ }^{6}$. First, $\beta^{T}=0$. Then, using a backwards

\footnotetext{
${ }^{6}$ Note that $\beta^{t}$ depends on the horizon $T$. When needed we write $\beta^{t}(T)$ instead of $\beta^{t}$.
} 
iteration, $\beta^{t}=H\left(\beta^{t+1}\right)$ for all $t=T-1, \ldots, 1$. We have (cf. Lemma 2.18.1)

$$
1>\beta^{1}>\beta^{2}>\cdots>\beta^{T}=0 .
$$

Proposition 2.2. Assume that the game has finite horizon $T$. Then the thresholds for the sender's strategy in the regular strategy profile are given by $\beta^{1}, \ldots, \beta^{T}$ respectively at periods $1, \ldots, T$.

Proof. Suppose that the players use a regular strategy profile $(\sigma, \tau)$. Assume that $\sigma$ has a threshold $\alpha^{t}$ at each period $t$. Then, at each period $t<T$, with probability $\alpha^{t}$ we have $\theta^{t}<\alpha^{t}$ and the receiver continues. In this case the sender gets the expected continuation payoff $U_{s}^{t+1}(\sigma, \tau)$. Similarly, with probability $1-\alpha^{t}$ we have $\theta^{t}>\alpha^{t}$ and the receiver quits. In this case the sender gets the expected continuation payoff $\frac{1}{1-\alpha^{t}} \int_{\alpha^{t}}^{1} f^{t}(\theta) d \theta$. As $\sigma$ is sincere against $\tau$, we have $f^{t}\left(\alpha^{t}\right)=U_{s}^{t+1}(\sigma, \tau)$ for $t=1, \ldots, T-1$. This yields the recursive equation

$$
\begin{aligned}
f^{t-1}\left(\alpha^{t-1}\right)= & U_{s}^{t}(\sigma, \tau)=\alpha^{t} \cdot U_{s}^{t+1}(\sigma, \tau)+\int_{\alpha^{t}}^{1} f^{t}(\theta) d \theta \\
& =\alpha^{t} \cdot f^{t}\left(\alpha^{t}\right)+\int_{\alpha^{t}}^{1} f^{t}(\theta) d \theta .
\end{aligned}
$$

Using that $f^{t}=\delta^{t-1} \cdot f$ in every period $t$, this can be rewritten to

$$
\alpha^{t-1}=f^{-1}\left(\delta \cdot\left[\alpha^{t} \cdot f\left(\alpha^{t}\right)+\int_{\alpha^{t}}^{1} f(\theta) d \theta\right]\right) .
$$

The last equation shows that $\alpha^{t-1}=H\left(\alpha^{t}\right)$ for $t=2, \ldots, T$. As the sender must send the message $m_{q}$ at period $T$ irrespective of the state, we have $\alpha^{T}=0$. So, we have $\alpha^{T}=\beta^{T}$ and inductively, $\alpha^{t-1}=$ $H\left(\alpha^{t}\right)=H\left(\beta^{t}\right)=\beta^{t-1}$ for $t=T, \ldots, 2$.

The numbers $\beta^{1}, \ldots, \beta^{T}$ can be computed recursively. Hence the regular strategy profile is entirely computable. It is worth noting that the computation of the regular strategy profile only considers the sender's 
Chapter 2. Incentive compatibility in sender-receiver stopping games

payoff function; the receiver's payoff function does not play any role at all.

\subsubsection{Infinite horizon}

The function $H$ has a unique fixed point, which is denoted by $\beta$ (ref. Lemma 2.17.2).

Proposition 2.3. Assume that the game has infinite horizon. Then there is a unique regular strategy profile. Moreover, this profile is stationary with threshold $\beta$.

Proof. Let $(\sigma, \tau)$ be a regular strategy profile. First note that the thresholds $\alpha^{t}$ of the regular strategy profile satisfy the formula $\alpha^{t}=$ $H\left(\alpha^{t+1}\right)$ for all $t$.

We first show that, for each period $t \in \mathbb{N}$ we have $\alpha^{t} \leq \beta$. Assume by way of contradiction that for some $t \in \mathbb{N}, \alpha^{t}>\beta$. We have $\alpha^{t}=$ $H\left(\alpha^{t+1}\right)$. By Lemma 2.17.3, $H\left(\alpha^{t}\right)<\alpha^{t}=H\left(\alpha^{t+1}\right)$. This implies $\alpha^{t}<$ $\alpha^{t+1}$. In particular, $\alpha^{t+1}>\beta$, and we can conclude that the sequence $\left(\alpha^{t^{\prime}}\right)_{t^{\prime}=t}^{\infty}$ is strictly increasing.

By definition, $\alpha^{t} \leq 1$ for each $t \in \mathbb{N}$. Moreover, $\alpha^{t^{\prime}}=H\left(\alpha^{t^{\prime}+1}\right) \leq$ $H(1)<1$ for each $t^{\prime}>t$. Hence, the sequence converges, say to $r<1$. Write

$$
z=\min \left\{x-H(x) \mid x \in\left[\alpha^{t}, 1\right]\right\} .
$$

By Lemma 2.17.3, $H(x)<x$ for $x \geq \alpha^{t}>\beta$. So, by continuity of $H$, we know that $z>0$. As the sequence $\left(\alpha^{t^{\prime}}\right)_{t^{\prime}=t}^{\infty}$ converges to $r$, for $\epsilon=\frac{z}{2}$, there exists $N>0$ such that $\alpha^{t^{\prime}} \in[r-\epsilon, r]$ for all $t^{\prime}>N$. Then for any $t^{\prime}>N$, it holds that

$$
\alpha^{t^{\prime}+1} \geq H\left(\alpha^{t^{\prime}+1}\right)+z=\alpha^{t^{\prime}}+z \geq r-\epsilon+z=r+\frac{z}{2} .
$$

This contradicts the fact that $\alpha^{t^{\prime}+1}>\beta$. Hence, $\alpha^{t} \leq \beta$ for each $t \in \mathbb{N}$. 
We show that $\alpha^{t}=\beta$ for each $t \in \mathbb{N}$. Assume that $\alpha^{t}<\beta$ for some $t$. As $\alpha^{t}=H\left(\alpha^{t+1}\right)$, by Lemma 2.17.3, $\alpha^{t+1}<\beta$. So, the sequence $\left(\beta^{t}\right)_{t=1}^{\infty}$ is decreasing and bounded below by 0 . Hence, the sequence converges, say to $r \geq 0$.

Take $\varepsilon=\frac{1}{2}(H(r)-r)$. As before, we can conclude that $\varepsilon>0$. As the sequence $\left(\alpha^{t}\right)_{t=1}^{\infty}$ converges to $r$, we can find $N>0$ such that $\alpha^{N}-r<$ $\epsilon$. Also, as the sequence is decreasing, $r<\alpha^{N+1}$ and hence $H(r)<$ $H\left(\alpha^{N+1}\right)$. So, we have

$$
H(r)-r<H\left(\alpha^{N+1}\right)-r=\alpha^{N}-r<\epsilon=\frac{1}{2}(H(r)-r)
$$

This is a contradiction. Hence, $\alpha^{t} \geq \beta$. By the previous argument, it follows that $\alpha^{t}=\beta$.

The fixed point $\beta$ can be computed by solving $H(x)=x$. Hence the regular strategy profile is entirely computable. As in the finite horizon, the computation of the regular strategy profile only considers the sender's payoff function; the receiver's payoff function does not play any role at all.

By Lemma 2.18.3. we have $\beta^{t}(T) \rightarrow \beta$ as $T \rightarrow \infty$ for each $t$. So the following corollary is immediate consequence of Propositions 2.2 and 2.3 .

Corollary 2.4. Let $\left(\sigma_{T}, \tau_{T}\right)$ be the regular strategy profile in the game with finite horizon $T$ and $(\sigma, \tau)$ be the regular strategy profile in the game with infinite horizon. Then the sequence $\left(\sigma_{T}, \tau_{T}\right)_{T=1}^{\infty}$ converges to $(\sigma, \tau)$ as $T \rightarrow$ $\infty$, when the payoffs are discounted or undiscounted.

\subsection{Existence and unicity of PBE, finite horizon}

In this section we consider the case where the game has some finite horizon $T$. We provide a existence and unicity result for the PBE of the game. The result shows that, for any finite horizon, if the payoffs 
are period-independent or they are discounted with a large discount factor, then the regular strategy profile is the unique responsive PBE. This means that the strategy profile in which the receiver is obedient and the sender is being sincere against this strategy of the receiver is the only PBE that is responsive. We also show that existence of PBE may fail for small discount factors.

Define the function $V:[0,1] \rightarrow \mathbb{R}$ by

$$
V(x)=\frac{1}{x} \cdot \int_{0}^{x} g(\theta) d \theta
$$

and $V(0)=0$. The amount $V(x)$ is the expected payoff for the receiver if he quits, conditional on the state being in $[0, x]$. For $T \in \mathbb{N}$, let $D^{T}$ be the smallest number ${ }^{7}$ in $[0,1]$ such that $\delta \cdot V(1) \geq V\left(\beta^{1}(T)\right)$ for every $\delta \in\left[D^{T}, 1\right]$.

Theorem 2.5. Consider a sender-receiver stopping game with finite horizon $T$. Let the payoffs be either period-independent or discounted with discount factor $\delta \geq D^{T}$. Then, the regular strategy profile is the unique responsive PBE.

Let $D$ be the smallest number in $[0,1]$ such that $\delta \cdot V(1) \geq V(\beta)$ for every $\delta \in[D, 1]$. If $f$ is Lipschitz at 1 then $D<1$, as is shown by Lemma 2.19.2. Notice that if $\delta \geq D$, then the existence and unicity holds regardless the horizon $T$ of the game.

Theorem 2.6. Suppose that $0<\delta<D^{2}$. Then, for any $T \geq 2$, the senderreceiver game with finite horizon $T$ does not admit a responsive $P B E$.

In these theorems, whether the regular strategy profile is a PBE (Theorem 2.5) or not (Theorem 2.6) depends on whether the receiver is patient enough, that is, whether the discount factor is sufficiently high. In

\footnotetext{
${ }^{7}$ The number $\beta^{1}(T)$ itself depends on the discount factor $\delta$. Hence, $D^{T}$ does not have a closed formula. It turns out that $D^{T}<1$.
} 
particular, it depends on whether he is willing to obey the sender and continue when the state is small. How high the discount factor should be, depends on the curvature of the receiver's payoff function and the thresholds set by the sender.

In the following example, if the discount factor is small then receiver is not inclined to obey the sender at period $T-1$ when the state is small. Hence, the regular strategy profile is not a PBE.

Example 2.7. Consider the game with finite horizon $T$ in which the payoff functions are $\delta$ discounted with $f(\theta)=\theta^{2}$ and $g(\theta)=\theta$. Then

$$
H(x)=\sqrt{\frac{\delta \cdot\left(1+2 x^{3}\right)}{3}} .
$$

If the message sent by the sender at period $T-1$ is $m_{c}$, then the state is in the interval $\left[0, \beta^{T-1}\right]$. So, the payoff for the receiver on quitting is $\delta^{T-2} \cdot V\left(\beta^{T-1}\right)$ and the payoff on continuing is $\delta^{T-1} \cdot V(1)$. Hence, the receiver prefers to continue if $\delta \cdot V(1) \geq V\left(\beta^{T-1}\right)$. We have $\beta^{T-1}=\sqrt{\frac{\delta}{3}}$. Further, $V(x)=\frac{x}{2}$. Thus, the inequality $\delta \cdot V(1) \geq V\left(\beta^{T-1}\right)$ is valid if and only if $\delta \geq \frac{1}{3}$. So, we get $D^{2}=\frac{1}{3}>0$. Hence, if $\delta<\frac{1}{3}$, the regular strategy profile is not a PBE. By Theorem 2.6, if $\delta<\frac{1}{3}$ then the game does not admit a responsive PBE.

\subsection{Existence and unicity of PBE, infinite horizon}

Now we consider sender-receiver games with infinite horizon. Recall that if the receiver never quits then both players get payoff zero. The payoffs are either period-independent or they are discounted. For the discounted case with sufficiently large discount factors, we prove the existence of a unique responsive PBE, which turns out to be stationary. Then, we show that if the payoffs are period-independent then a PBE fails to exist. 
Chapter 2. Incentive compatibility in sender-receiver stopping games

Theorem 2.8. Consider a sender-receiver stopping game with infinite horizon in which the payoffs are discounted with discount factor $\delta \geq D$. Then the regular strategy profile is the unique responsive PBE among the essentially Markov strategy profiles.

Note that, by Proposition 2.3, the regular strategy profile is stationary, with threshold $\beta$. Now we turn to period-independent payoffs.

Theorem 2.9. Consider a sender-receiver stopping game with infinite horizon in which the payoffs are period-independent. Then, there exists no responsive PBE within essentially Markov strategy profiles.

The result of theorem 2.9 is driven by the following observation. At period 1, it has probability 1 that a state strictly less than 1 is realized. Since the horizon of the game is infinite, the sender knows that if both players wait sufficiently long, then a strictly better state will be realized later on. Hence, he would like the game to continue. Since this argument holds for each period, the sender is never satisfied. In fact, the players can get payoffs arbitrarily close to $f(1)$ and $g(1)$, but with probability 1 they can not get them exactly. Note that in the regular strategy profile, the threshold in the sender's strategy would be 1 at each period.

The following Corollary is the immediate consequence of Corollary 2.4 and Theorems 2.5, 2.8 and 2.9.

Corollary 2.10. If the payoffs are discounted $(\delta<1)$, the sequence of PBEs in the finite horizon games converges to a $P B E$ in the infinite horizon game. If the payoffs are undiscounted $(\delta=1)$, the sequence of PBE in the finite horizon games converges to the regular strategy profile in the infinite horizon game which is not a PBE. 


\subsection{Examples}

In this section we illustrate our results with the help of the examples. We consider a sender-receiver stopping game in which the payoffs are either period-independent $(\delta=1)$ or discounted with discount factor $\delta \in(0,1)$. As discussed before, the regular strategy profile is determined solely by the payoffs of the sender.

Example 2.11. $f(x)=x^{2}$ and $g(x)=x$. The function $H$ can be calculated as $H(x)=\sqrt{\delta \cdot \frac{1+2 x^{3}}{3}}$.

We first consider the setting in which the game has a finite horizon $T$. By definition, we have $\beta^{T}(T)=0$ and for each $t<T, \beta^{t}(T)=$ $H\left(\beta^{t+1}(T)\right)$. By Proposition 2.2, the game has a unique regular strategy profile. In this profile, the receiver plays the obeying strategy and the sender plays the sincere strategy (against the receiver's strategy) with threshold $\beta^{t}(T)$ at period $t$.

Now as $g(x)=x$, the function $V$ can be calculated as $V(x)=\frac{x}{2}$. Recall from Section 2.4 that the bound $D^{T}$ is defined as the smallest number such that $\delta \cdot V(1) \geq V\left(\beta^{1}(T)\right)$ for $\delta \in\left[D^{T}, 1\right]$. The inequality simplifies to $\delta \geq \beta^{1}(T)$.

If the horizon $T=3$, then $\beta^{3}(3)=0, \beta^{2}(3)=\sqrt{\frac{\delta}{3}}$ and $\beta^{1}(3)=$ $\sqrt{\frac{3 \sqrt{3} \cdot \delta+2 \cdot \delta^{2.5}}{9 \sqrt{3}}}$. The inequality $\delta \geq \beta^{1}(3)$ is satisfied if and only if $\delta \in$ $\left[D^{3}, 1\right]$ and in this example, $D^{3} \approx 0.361$. Hence, if $\delta \in\left[D^{3}, 1\right]$, the regular strategy profile is a unique responsive PBE (ref. Theorem 2.5). If $T=2$, then $\beta^{1}(2)=\sqrt{\frac{\delta}{3}}$ and $D^{2}=\frac{1}{3}$. So, the regular strategy profile is a unique responsive PBE for $\delta \in\left[D^{2}, 1\right]$. For $T \geq 2$, if $\delta \in\left(0, D^{2}\right)$, the game has no responsive PBE among the essentially Markov strategy profiles (ref. Theorem 2.6).

Now we consider the setting in which the game has infinite horizon. By Proposition 2.3, the game has a unique regular strategy profile. In this profile, the receiver plays the obeying strategy and the sender 
Chapter 2. Incentive compatibility in sender-receiver stopping games

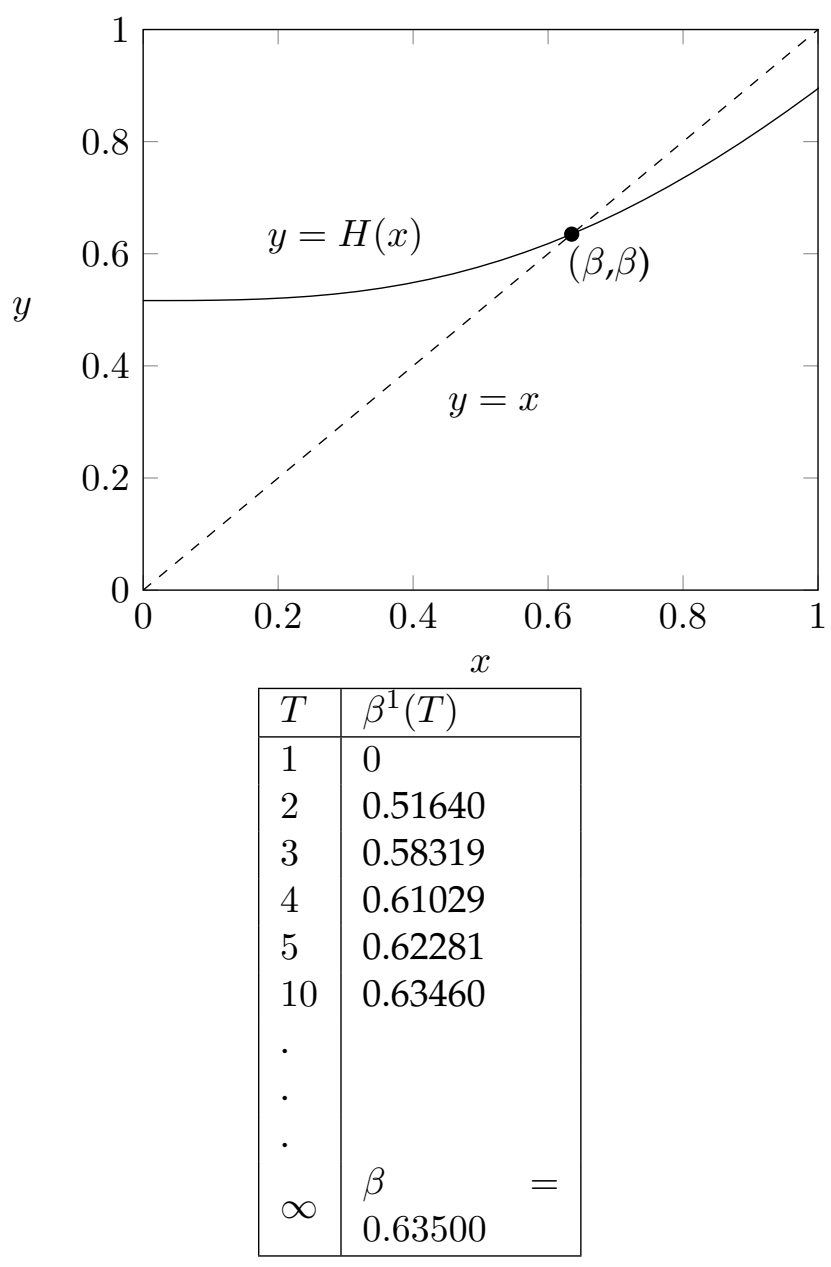

Figure 2.1: $f(x)=x^{2}, \delta=0.8$

plays the sincere strategy (against the receiver's strategy) with threshold $\beta$ at each period $t$. Here $\beta$ is the unique solution in $[0,1]$ to the equation $H(x)=x$.

We have $H(\beta)=\beta$, which can be rewritten as $\delta=\frac{3 \beta^{2}}{1+2 \beta^{3}}$. For $\delta<1$, 
we have $\beta<1$. Recall from Section 2.5 that the bound $D$ is defined as the smallest number such that $\delta \cdot V(1) \geq V(\beta)$ for $\delta \in[D, 1]$. The inequality is equivalent to $\delta \geq \beta$. This holds if and only if $\delta \in[D, 1]$. In this example, $D$ solves the equation $2 \delta x^{3}-3 x^{2}+\delta=0$. Hence, $D \approx 0.366$. So, if $\delta \in[D, 1)$, the regular strategy profile is a unique responsive PBE (ref. Theorem 2.8).

If the payoffs are period independent, i.e. $\delta=1$, then by Theorem 2.9 , there is no responsive PBE among the essentially Markov strategy profiles. Indeed, in this case, we have $\beta=1$. So, according to the regular strategy profile, the sender sends the message $m_{c}$ whenever the state is less than 1 and the receiver obeys. Hence, the game is played forever with probability 1 , and the expected payoff is 0 for both the players.

Example 2.12. Here we assume $f(x)=x^{2}$ and $g(x)=x^{3}$. As the payoff function of the sender is same as in Example 1, so is the unique regular strategy profile. As $g(x)=x^{3}$, we have $V(x)=\frac{x^{3}}{4}$. In this case, we have $D^{T}=0$ for each $T \geq 1$ and $D=0$. Hence, in the setting of the game with a finite horizon $T$ (for $T \geq 1$ ), the regular strategy profile is the unique responsive PBE for $\delta \in(0,1]$ and in the setting of the game with the infinite horizon, the regular strategy profile is the unique responsive $\mathrm{PBE}$ for $\delta \in(0,1)$.

\subsection{Concluding remarks}

This chapter shows that the model of sender-receiver stopping games differs from the other models of dynamic sender-receiver games in the literature. The striking feature about this model stated by our main results is that under the responsive PBE the sender plays the threshold strategy optimal for himself and the receiver simply obeys. This is surprising, as the receiver has to comply with the sender regardless his own payoff function. Under the responsive PBE the sender gets 
the maximum possible payoff for himself. Hence, the delegation of the decision making to the receiver does not hurt the sender.

We see many interesting open question to be addressed in future work. One immediate question would be the extension to arbitrary distributions of the state of the world. The simple case in which nature draws an iid state from a strictly increasing continuous distribution is discussed in Appendix C. A challenging future extension would be the case in which the state of the world follows a Markov chain. We are currently investigating the situation with multiple senders, so that the receiver can make better informed decisions.

\subsection{The proofs}

\subsubsection{The proof of Theorem 2.5}

We will prove Theorem 2.5 in two parts: Claim 2.13 and Claim 2.14. So we fix a sender-receiver stopping game with finite horizon $T$, and with payoffs that are period-independent $(\delta=1)$ or they are discounted with discount factor $\delta \in(0,1)$.

Claim 2.13. Let $\delta \in\left[D^{T}, 1\right]$. Suppose that $\tau$ is responsive. If the strategy profile $(\sigma, \tau)$ is a PBE, then $(\sigma, \tau)$ is regular.

Proof. Fix $\delta \in\left[D^{T}, 1\right]$. Suppose that $(\sigma, \tau)$ is a PBE with $\tau$ being responsive. We will prove that $(\sigma, \tau)$ is regular. We shall do so by proving that the sender's strategy $\sigma$ is sincere and the receiver's strategy $\tau$ is obedient at each period $t=1, \ldots, T-1$. We apply backward induction by considering the periods in the order $T-1, T-2, \ldots, 1$.

We recall from Section 2.2.2 that at period $t$, the expected payoffs $U_{s}^{t}(\sigma, \tau)$ and $U_{r}^{t}(\sigma, \tau)$ are functions of the histories of the sender and the receiver respectively.

For $t=T-1, T-2, \ldots, 1$, let $Q(t)$ be the following list of statements: 
[1] $\sigma$ is sincere at period $t$, and the corresponding threshold is equal to $\beta^{t}$.

[2] $U_{r}^{t+1}(\sigma, \tau)>\frac{1}{\beta^{t}} \int_{0}^{\beta^{t}} g^{t}(\theta) d \theta$.

[3] $U_{r}^{t+1}(\sigma, \tau)<\frac{1}{1-\beta^{t}} \cdot \int_{\beta^{t}}^{1} g^{t}(\theta) d \theta$.

[4] $\tau$ is obedient at period $t$. That is, for each history $h_{r}^{t}, \tau^{t}\left(h_{r}^{t}, m_{c}\right)=$ 1 and $\tau^{t}\left(h_{r}^{t}, m_{q}\right)=0$.

[5] $U_{s}^{t}(\sigma, \tau)$ and $U_{r}^{t}(\sigma, \tau)$ do not depend on history up to period $t$.

For ease of notation, and suppressing possible history dependence, for every period $t=1, \ldots, T$ let $p^{t}=\tau^{t}\left(h_{r}^{t}, m_{c}\right)$ be the probability on the action $a_{c}$ on seeing the message $m_{c}$, and let $q^{t}=\tau^{t}\left(h_{r}^{t}, m_{q}\right)$ be the probability on the action $a_{c}$ on seeing the message $m_{q}$. As $\tau$ is responsive, we know that $p^{t}>q^{t}$.

Remark on period $T$. The final period is a special case. At this period the sender always sends message $m_{q}$ and the receiver always chooses action $a_{q}$. So, $U_{s}^{T}(\sigma, \tau)$ and $U_{r}^{T}(\sigma, \tau)$ are independent of the history up to period $T$.

The induction step. We assume that $Q(t+1), \ldots, Q(T-1)$ are true, for some $t \in\{1, \ldots, T-1\}$. We show that $Q(t)$ is also true.

Item [1] of $\mathbf{Q}(\mathbf{t})$. First we show that $\beta^{t}$ is the (unique) solution to the equation $f^{t}(x)=U_{s}^{t+1}(\sigma, \tau)$. For $t=T-1$ we have

$$
\begin{aligned}
U_{s}^{T}(\sigma, \tau) & =\int_{0}^{1} f^{T}(\theta) d \theta \\
& =\delta^{T-1} \cdot \int_{0}^{1} f(\theta) d \theta=\delta^{T-2} \cdot f(H(0)) \\
& =\delta^{T-2} \cdot f\left(H\left(\beta^{T}\right)\right)=\delta^{T-2} \cdot f\left(\beta^{T-1}\right) \\
& =f^{T-1}\left(\beta^{T-1}\right) .
\end{aligned}
$$


Chapter 2. Incentive compatibility in sender-receiver stopping games

Now consider the case when $t \leq T-2$. By item [5] of $Q(t+1)$, the expected payoff $U_{s}^{t+1}(\sigma, \tau)$ is independent of the history up to period $t+1$. By item [5] of $Q(t+2)$ when $t \leq T-3$ and by the remark on period $T$ when $t=T-2$, the expected payoff $U_{s}^{t+2}(\sigma, \tau)$ is independent of the history up to period $t+2$. We have

$$
\begin{aligned}
U_{s}^{t+1}(\sigma, \tau) & =\beta^{t+1} \cdot U_{s}^{t+2}(\sigma, \tau)+\int_{\beta^{t+1}}^{1} f^{t+1}(\theta) d \theta \\
& =\beta^{t+1} \cdot f^{t+1}\left(\beta^{t+1}\right)+\int_{\beta^{t+1}}^{1} f^{t+1}(\theta) d \theta \\
& =\delta^{t-1} \cdot \delta \cdot\left[\beta^{t+1} \cdot f\left(\beta^{t+1}\right)+\int_{\beta^{t+1}}^{1} f(\theta) d \theta\right] \\
& =\delta^{t-1} \cdot f\left(H\left(\beta^{t+1}\right)\right) \\
& =\delta^{t-1} \cdot f\left(\beta^{t}\right) \\
& =f^{t}\left(\beta^{t}\right) .
\end{aligned}
$$

For the first equality, we use items [1] and [4] of $Q(t+1)$. For the second equality, we use item [1] of $Q(t+1)$ and the definition of sincere strategy. For the fifth equality, we use the definition of $\beta^{t}$.

Thus, when $\theta^{t}<\beta^{t}$, the sender expects to get a strictly better payoff if the receiver continues than when the receiver quits. As $p^{t}>q^{t}$, the sender strictly prefers to send the message $m_{c}$ over $m_{q}$ if $\theta^{t}<\beta^{t}$. By the same reasoning, the sender strictly prefers to send the message $m_{q}$ over $m_{c}$ if $\theta^{t}>\beta^{t}$. Hence, $\sigma$ is sincere at period $t$ with threshold value $\beta^{t}$.

Item [2] of $\mathbf{Q}(\mathbf{t})$. Quitting at period $t+1$ guarantees payoff $\int_{0}^{1} g^{t+1}(\theta) d \theta$ for the receiver. So by using Lemma 2.18.2, we get

$$
\frac{1}{\beta^{t}} \cdot \int_{0}^{\beta^{t}} g^{t}(\theta) d \theta<\int_{0}^{1} g^{t+1}(\theta) d \theta \leq U_{r}^{t+1}(\sigma, \tau) .
$$

This proves item [2] of $Q(t)$. 
Item [3] of $\mathbf{Q}(\mathbf{t})$. For $t=T-1$, by using Lemma 2.20, we have $U_{r}^{T}(\sigma, \tau)=\int_{0}^{1} g^{T}(\theta) d \theta \leq \int_{0}^{1} g^{T-1}(\theta) d \theta<\frac{1}{1-\beta^{T-1}} \cdot \int_{\beta^{T-1}}^{1} g^{T-1}(\theta) d \theta$.

For $t<T-1$ we have

$$
\begin{aligned}
U_{r}^{t+1}(\sigma, \tau) & =\beta^{t+1} \cdot U_{r}^{t+2}(\sigma, \tau)+\int_{\beta^{t+1}}^{1} g^{t+1}(\theta) d \theta \\
& <\frac{1}{1-\beta^{t+1}} \cdot \int_{\beta^{t+1}}^{1} g^{t+1}(\theta) d \theta \\
& \leq \frac{1}{1-\beta^{t+1}} \cdot \int_{\beta^{t+1}}^{1} g^{t}(\theta) d \theta \\
& <\frac{1}{1-\beta^{t}} \cdot \int_{\beta^{t}}^{1} g^{t}(\theta) d \theta .
\end{aligned}
$$

In the equality, we use items [1] and [4] of $Q(t+1)$. In the first inequality, we use item [3] of $Q(t+1)$. For the last inequality, we use Lemmas 2.18.1 and 2.20.

Item [4] of $\mathbf{Q}(\mathbf{t})$. Assume the message is $m_{c}$ at period $t$. Then by item [1] of $Q(t)$, we have $\theta^{t} \in\left[0, \beta^{t}\right]$. We want to show that $\tau$ plays action $a_{c}$ at period $t$. The expected payoff for the receiver on quitting is $\frac{1}{\beta^{t}} \int_{0}^{\beta^{t}} g^{t}(\theta) d \theta$. By item [2] of $Q(t)$ we have

$$
U_{r}^{t+1}(\sigma, \tau)>\frac{1}{\beta^{t}} \int_{0}^{\beta^{t}} g^{t}(\theta) d \theta .
$$

Since $(\sigma, \tau)$ is a PBE, $\tau$ has to play action $a_{c}$ at period $t$.

Now, assume the message is $m_{q}$ at period $t$. Then by item [1] of $Q(t)$, we have $\theta^{t} \in\left[\beta^{t}, 1\right]$. We want to show that $\tau$ plays action $a_{q}$ at period $t$. The expected payoff for the receiver on quitting is $\frac{1}{1-\beta^{t}} \int_{\beta^{t}}^{1} g^{t}(\theta) d \theta$. 
Chapter 2. Incentive compatibility in sender-receiver stopping games

By [3] of $Q(t)$ we have

$$
U_{r}^{t+1}(\sigma, \tau)<\frac{1}{1-\beta^{t}} \int_{\beta^{t}}^{1} g^{t}(\theta) d \theta .
$$

Since $(\sigma, \tau)$ is a PBE, $\tau$ has to play action $a_{q}$ at period $t$.

Item [5] of $\mathbf{Q ( t )}$. By using items [1] and [4] of $Q(t), \ldots, Q(T-1)$ and the remark on period $T$, the statement follows immediately.

Claim 2.14. Let $\delta \in\left[D^{T}, 1\right]$. Then the regular strategy profile is a PBE.

Proof. Let $(\sigma, \tau)$ be regular. Then the expected payoffs $U_{s}^{t}(\sigma, \tau)$ and $U_{r}^{t}(\sigma, \tau)$ are independent of the history up to period $t-1$ for all periods $t=1, \ldots, T$. We show that $(\sigma, \tau)$ is a PBE in four steps.

Step 1. We show that, for all $t=1, \ldots, T$, the threshold used by $\sigma$ at period $t$ is exactly $\beta^{t}$.

Proof of step 1. The proof is by backward induction. At period $T$, by definition, $\sigma$ uses threshold $\beta^{T}=0$.

Now assume that at some period $t=2, \ldots, T$, the strategy $\sigma$ uses threshold $\beta^{t}$. We argue that the threshold of $\sigma$ at period $t-1$ is $\beta^{t-1}$. We have

$$
\begin{aligned}
U_{s}^{t}(\sigma, \tau) & =\beta^{t} \cdot U_{s}^{t+1}(\sigma, \tau)+\int_{\beta^{t}}^{1} f^{t}(\theta) d \theta \\
& =\beta^{t} \cdot f^{t}\left(\beta^{t}\right)+\int_{\beta^{t}}^{1} f^{t}(\theta) d \theta \\
& =\delta^{t-1} \cdot\left[\beta^{t} \cdot f\left(\beta^{t}\right)+\int_{\beta^{t}}^{1} f(\theta) d \theta\right] \\
& =\delta^{t-1} \cdot G\left(\beta^{t}\right)=\delta^{t-2} \cdot f\left(H\left(\beta^{t}\right)\right) \\
& =\delta^{t-2} \cdot f\left(\beta^{t-1}\right)=f^{t-1}\left(\beta^{t-1}\right) .
\end{aligned}
$$


In the first equality, we use that $\sigma$ uses threshold $\beta^{t}$ and $\tau$ is obedient at period $t$. In the second equality, we use that $\sigma$ is sincere at period $t$. In the sixth equality, we use the definition of $\beta^{t-1}$.

Since $\sigma$ is sincere at period $t-1$, the threshold at period $t-1$ must be $\beta^{t-1}$, as desired. This completes the proof of step 1 .

Step 2. For each period $t=1, \ldots, T-1$, let $Q(t)$ be the statement that the following conditions hold:

[1] If the receiver gets the message $m_{q}$ at period $t$, then

$$
U_{r}^{t+1}(\sigma, \tau)<\frac{1}{1-\beta^{t}} \cdot \int_{\beta^{t}}^{1} g^{t}(\theta) d \theta
$$

[2] If the receiver gets the message $m_{c}$ at period $t$, then

$$
U_{r}^{t+1}(\sigma, \tau)>\frac{1}{\beta^{t}} \int_{0}^{\beta^{t}} g^{t}(\theta) d \theta
$$

We prove that $Q(t)$ holds for all $t=1, \ldots, T-1$.

Proof of step 2. The proof is by backward induction. First we consider period $T-1$. We have by Lemma 2.20

$U_{r}^{T}(\sigma, \tau)=\int_{0}^{1} g^{T}(\theta) d \theta \leq \int_{0}^{1} g^{T-1}(\theta) d \theta<\frac{1}{1-\beta^{T-1}} \cdot \int_{\beta^{T-1}}^{1} g^{T-1}(\theta) d \theta$,

which proves item [1] of the statement $Q(T-1)$. Item [2] of the statement $Q(T-1)$ follows from Lemma 2.18.2.

Now assume that $Q(t+1)$ is true, where $t \in\{1, \ldots, T-2\}$. We prove that $Q(t)$ also holds. 
Chapter 2. Incentive compatibility in sender-receiver stopping games

First we prove the item [1] of $Q(t)$. We have

$$
\begin{aligned}
U_{r}^{t+1}(\sigma, \tau) & =\beta^{t+1} \cdot U_{r}^{t+2}(\sigma, \tau)+\int_{\beta^{t+1}}^{1} g^{t+1}(\theta) d \theta \\
& \leq \beta^{t+1} \cdot \frac{1}{1-\beta^{t+1}} \cdot \int_{\beta^{t+1}}^{1} g^{t+1}(\theta) d \theta+\int_{\beta^{t+1}}^{1} g^{t+1}(\theta) d \theta \\
& =\frac{1}{1-\beta^{t+1}} \cdot \int_{\beta^{t+1}}^{1} g^{t+1}(\theta) d \theta \\
& \leq \frac{1}{1-\beta^{t+1}} \cdot \int_{\beta^{t+1}}^{1} g^{t}(\theta) d \theta \\
& <\frac{1}{1-\beta^{t}} \cdot \int_{\beta^{t}}^{1} g^{t}(\theta) d \theta .
\end{aligned}
$$

In the first equality, we use that, by step $1, \sigma$ uses threshold $\beta^{t+1}$ and $\tau$ is obedient at period $t+1$. In the first inequality, we use item [1] of $Q(t+1)$. In the last inequality, we use Lemma 2.20 and the fact that $\beta^{t}>\beta^{t+1}$. This completes the proof of item [1] of $Q(t)$.

Now we prove the item [2] of $Q(t)$. We have

$$
\begin{aligned}
U_{r}^{t+1}(\sigma, \tau) & =\beta^{t+1} \cdot U_{r}^{t+2}(\sigma, \tau)+\int_{\beta^{t+1}}^{1} g^{t+1}(\theta) d \theta \\
& \geq \beta^{t+1} \cdot \frac{1}{\beta^{t+1}} \cdot \int_{0}^{\beta^{t+1}} g^{t+1}(\theta) d \theta+\int_{\beta^{t+1}}^{1} g^{t+1}(\theta) d \theta \\
& =\int_{0}^{1} g^{t+1}(\theta) d \theta \\
& >\frac{1}{\beta^{t}} \cdot \int_{0}^{\beta^{t}} g^{t}(\theta) d \theta .
\end{aligned}
$$

In the first equality, we use that, by step $1, \sigma$ uses threshold $\beta^{t+1}$ and $\tau$ is obedient at period $t+1$. In the first inequality, we use item [2] of $Q(t+1)$. In the second inequality, we use the Lemma 2.18.2. This completes the proof of item [2] of $Q(t)$.

Step 3. We prove that, starting from any period $t=1, \ldots, T$, the obe- 
dient strategy $\tau$ of the receiver is a best response against the sincere strategy $\sigma$ of the sender.

Proof of step 3. At any period $t \in\{1, \ldots, T-1\}$, consider the case where the receives gets message $m_{q}$. We show that it is a (unique) best response for the receiver to play $a_{q}$. That is, $\tau$ plays a best response at period $t$.

Because the receiver gets the message $m_{q}$ at period $t$, we have by step 1 that $\theta^{t} \in\left[\beta^{t}, 1\right]$. So, the expected payoff for the receiver on quitting is $\frac{1}{1-\beta^{t}} \int_{\beta^{t}}^{1} g^{t}(\theta) d \theta$. By step 2, we have

$$
U_{r}^{t+1}(\sigma, \tau)<\frac{1}{1-\beta^{t}} \cdot \int_{\beta^{t}}^{1} g^{t}(\theta) d \theta .
$$

Hence, it is a best response for the receiver to play $a_{q}$.

Now at any period $t \in\{1, \ldots, T-1\}$, consider the case where the receives gets message $m_{c}$. We show that it is a (unique) best response for the receiver to play $a_{c}$. That is, $\tau$ plays a best response at period $t$.

Because the receiver gets the message $m_{c}$ at period $t$, we have by step 1 that $\theta^{t} \in\left[0, \beta^{t}\right]$. So, the expected payoff for the receiver on quitting is $\frac{1}{\beta^{t}} \int_{0}^{\beta^{t}} g^{t}(\theta) d \theta$. By step 2, we have

$$
U_{r}^{t+1}(\sigma, \tau)>\frac{1}{\beta^{t}} \cdot \int_{0}^{\beta^{t}} g^{t}(\theta) d \theta .
$$

Hence, it is a best response for the receiver to play $a_{c}$.

Step 4. As the final step, we argue that $(\sigma, \tau)$ is a PBE.

Proof of step 4. By step 3, at any period, $\tau$ is best response against $\sigma$. Conversely, we show that $\sigma$ is a best response against $\tau$. Recall that for each period $t=1, \ldots, T-1$, the expected payoff $U_{s}^{t+1}(\sigma, \tau)$ is history independent. Since $\tau$ is obedient, the sender receives payoff $f^{t}\left(\theta^{t}\right)$ if 
$\sigma$ sends $m_{q}$ at period $t$ and payoff $U_{s}^{t+1}(\sigma, \tau)$ if $\sigma$ sends $m_{c}$. So, the sender plays a best response at period $t$ when he sends $m_{c}$ if $f^{t}\left(\theta^{t}\right)<$ $U_{s}^{t+1}(\sigma, \tau)$ and $m_{q}$ if $f^{t}\left(\theta^{t}\right)>U_{s}^{t+1}(\sigma, \tau)$. Hence, since $U_{s}^{t+1}(\sigma, \tau)=$ $f^{t}\left(\beta^{t}\right)$, the sender plays a best response at period $t$ when he sends $m_{c}$ if $\theta^{t}<\beta^{t}$ and $m_{q}$ if $\theta^{t}>\beta^{t}$. Thus, by step 1 , the strategy $\sigma$ is a best response against $\tau$ at period $t$. This completes the proof of the theorem.

\subsubsection{The proof of Theorem 2.6}

In this section we prove Theorem 2.6. So, let $f$ and $g$ be strictly increasing functions from $I$ to $\mathbb{R}_{+}$with $f(0)=g(0)=0$. Consider a finite horizon $T \geq 2$. Assume by way of contradiction that a strategy profile $(\sigma, \tau)$ is a $\operatorname{PBE}$ where $\tau$ is a responsive strategy.

As the receiver must quit at period $T$, we have $U_{s}^{T}(\sigma, \tau)=\int_{0}^{1} \delta^{T-1} f(\theta) d \theta$. Let $\quad \alpha^{T-1}=f^{-1}\left(\delta \cdot \int_{0}^{1} f(\theta) d \theta\right)$.

At period $T-1$, if the receiver quits, the sender obtains the payoff $f^{T-1}\left(\theta^{T-1}\right)$ and if the receiver continues, the sender obtains the expected payoff $U_{s}^{T}(\sigma, \tau)$. As $\tau$ is responsive, the sender strictly prefers to send the message $m_{c}$ if $\theta^{T-1}<\alpha^{T-1}$ and the message $m_{q}$ if $\theta^{T-1}>\alpha^{T-1}$. Hence, the strategy $\sigma$ has a threshold $\alpha^{T-1}$ at period $T-1$.

Assume that the receiver gets the message $m_{c}$ at period $T-1$. As $\alpha^{T-1}>0$, this happens with positive probability. If the receiver continues, he obtains the expected payoff

$$
U_{r}^{T}(\sigma, \tau)=\int_{0}^{1} \delta^{T-1} g(\theta) d \theta=\delta^{T-1} \cdot V(1) .
$$

And if the receiver quits, he obtains the expected payoff

$$
\frac{1}{\alpha^{T-1}} \int_{0}^{\alpha^{T-1}} \delta^{T-2} g(\theta) d \theta=\delta^{T-2} \cdot V\left(\alpha^{T-1}\right) .
$$


As $\delta<D^{2}$, we have $\delta \cdot V(1)<V\left(\alpha^{T-1}\right)$. So, the receiver strictly prefers to quit on receiving the message $m_{c}$. This is however in contradiction with $\tau$ being responsive. So, the game admits no responsive PBE.

\subsubsection{The proof of Theorem 2.8}

We will prove Theorem 2.8 in two parts: Claim 2.15 and Claim 2.16. So we fix a sender-receiver stopping game with infinite horizon and with payoffs that are discounted with discount factor $\delta \in(0,1)$.

Claim 2.15. Suppose that $\delta \in(D, 1)$. Let $(\sigma, \tau)$ be an essentially Markov $P B E$ where $\tau$ is responsive. Then, $(\sigma, \tau)$ is the regular strategy profile.

Proof. Suppose that $\delta \in(D, 1)$. Let $(\sigma, \tau)$ be an essentially Markov $\operatorname{PBE}$ where $\tau$ is responsive.

Again, let $p^{t}=\tau^{t}\left(h_{r}^{t}, m_{c}\right)$ be the probability on the action $a_{c}$ on seeing the message $m_{c}$, and let $q^{t}=\tau^{t}\left(h_{r}^{t}, m_{q}\right)$ be the probability on the action $a_{c}$ on seeing the message $m_{q}$. Since $\tau$ is responsive, $p^{t}>q^{t}$ for each $t$. We prove the statement in a series of steps.

Step 1. The strategy $\sigma$ has threshold $\alpha^{t}$ at each period $t \in \mathbb{N}$ that satisfies $f^{t}\left(\alpha^{t}\right)=U_{s}^{t+1}(\sigma, \tau)$. Hence, $\sigma$ is sincere.

Proof of step 1. Fix a period $t \in \mathbb{N}$. Since $(\sigma, \tau)$ is essentially Markov, $U_{s}^{t+1}(\sigma, \tau)$ is independent of the history up to period $t+1$. Further, note that $f^{t}(1)>f^{t+1}(1) \geq U_{s}^{t+1}(\sigma, \tau) \geq 0=f^{t}(0)$. So, the equation $f^{t}(x)=U_{s}^{t+1}(\sigma, \tau)$ has the unique solution, say $\alpha^{t} \in[0,1)$.

Assume first that $\theta^{t}<\alpha^{t}$. Then, $f^{t}\left(\theta^{t}\right)<f^{t}\left(\alpha^{t}\right)=U_{s}^{t+1}(\sigma, \tau)$. Thus, the sender expects to get a strictly better payoff when the receiver continues than when the receiver quits. As $p^{t}>q^{t}$, the sender strictly prefers to send the message $m_{c}$ over $m_{q}$. Because $(\sigma, \tau)$ is a PBE, $\sigma$ sends the message $m_{c}$ at period $t$. 
Chapter 2. Incentive compatibility in sender-receiver stopping games

Assume now that $\theta^{t}>\alpha^{t}$. Then, $f^{t}\left(\theta^{t}\right)>f^{t}\left(\alpha^{t}\right)=U_{s}^{t+1}(\sigma, \tau)$. By the same reasoning, $\sigma$ sends the message $m_{q}$ at period $t$. Thus, $\sigma$ is sincere at period $t$ with threshold $\alpha^{t}$. This completes the proof of step 1.

Step 2. Define for each period $t \in \mathbb{N}$

$$
K(t)=\frac{1}{\alpha^{t}} \cdot \int_{0}^{\alpha^{t}} g^{t}(\theta) d \theta \quad \text { and } \quad L(t)=\frac{1}{1-\alpha^{t}} \cdot \int_{\alpha^{t}}^{1} g^{t}(\theta) d \theta
$$

Then, for every $t \in \mathbb{N}$ we have $K(t) \leq U_{r}^{t+1}(\sigma, \tau) \leq L(t)$.

Proof of step 2. Fix $t \in \mathbb{N}$. As $\tau$ is responsive, we know that $1 \geq$ $p^{t}>q^{t} \geq 0$. We know that at period $t$, the strategy $\sigma$ uses threshold $\alpha^{t}$. So, $K(t)$ is the expected payoff to the receiver when he quits, upon getting the message $m_{c}$. Since $p^{t}>0$, it follows that $K(t) \leq U_{r}^{t+1}(\sigma, \tau)$. Similarly, $L(t)$ is the expected payoff to the receiver when he quits, upon getting the message $m_{q}$. Since $q^{t}<1$, it follows that $U_{r}^{t+1}(\sigma, \tau) \leq$ $L(t)$. This completes the proof of step 2 .

Step 3. For each period $t \in \mathbb{N}$ we have $\alpha^{t} \leq \beta$.

Proof of Step 3. Fix $t \in \mathbb{N}$. Suppose by way of contradiction that $\alpha^{t}>\beta$. Note that

$$
\begin{aligned}
f^{t}\left(\alpha^{t}\right) & =U_{s}^{t+1}(\sigma, \tau) \\
& \leq \alpha^{t+1} \cdot U_{s}^{t+2}(\sigma, \tau)+\int_{\alpha^{t+1}}^{1} f^{t+1}(\theta) d \theta \\
& =\alpha^{t+1} \cdot f^{t+1}\left(\alpha^{t+1}\right)+\int_{\alpha^{t+1}}^{1} f^{t+1}(\theta) d \theta \\
& =\delta^{t} \cdot G\left(\alpha^{t+1}\right) .
\end{aligned}
$$

The first and second equalities follow from the definitions of $\alpha^{t}$ and $\alpha^{t+1}$ and the last equality from the definition of $G$. The inequality can be explained as follows. If $\theta^{t+1}<\alpha^{t+1}$, which has probability $\alpha^{t+1}$, then the sender's payoff is at most $U_{s}^{t+2}(\sigma, \tau)$ (which is realized if the 
receiver continues). If $\theta^{t+1}>\alpha^{t+1}$, which has probability $1-\alpha^{t+1}$, then the sender's payoff is at most $\frac{1}{1-\alpha^{t+1}} \int_{\alpha^{t+1}}^{1} f^{t+1}(\theta) d \theta$ (which is realized if the receiver quits).

It follows that $f\left(\alpha^{t}\right) \leq \delta \cdot G\left(\alpha^{t+1}\right)$. So, $\alpha^{t} \leq H\left(\alpha^{t+1}\right)$. As $\alpha^{t}>\beta$ by assumption, by Lemma 2.17.3, $H\left(\alpha^{t}\right)<\alpha^{t} \leq H\left(\alpha^{t+1}\right)$. This implies $\alpha^{t}<\alpha^{t+1}$ and so $\alpha^{t+1}>\alpha$. Repeating the whole argument for $t+1$, we get $\alpha^{t+2}>\alpha^{t+1}>\beta$. And hence iterating the argument, we can conclude that the sequence $\left(\alpha^{t^{\prime}}\right)_{t^{\prime}=t}^{\infty}$ is strictly increasing. By definition, $\alpha^{t} \leq 1$ for each $t \in \mathbb{N}$. Moreover, $\alpha^{t^{\prime}} \leq H\left(\alpha^{t^{\prime}+1}\right) \leq H(1)$ for each $t^{\prime}>t$. Hence, the sequence $\left(\alpha^{t^{\prime}}\right)_{t^{\prime}=t}^{\infty}$ converges to some $r \leq H(1)<1$.

Denote $z=\min \left\{x-H(x): x \in\left[\alpha^{t}, 1\right]\right\}$. By Lemma 2.17.3, $H(x)<x$ for $x \geq \alpha^{t}>\beta$. By continuity of $H$, we have $z>0$. As the sequence $\left(\alpha^{t^{\prime}}\right)_{t^{\prime}=t}^{\infty}$ converges to $r$, for $\epsilon=\frac{z}{2}$, there exists $N>0$ such that $\alpha^{t^{\prime}} \in$ $[r-\epsilon, r]$ for all $t^{\prime}>N$. Now for any fixed $t^{\prime}>N$, we have $\alpha^{t^{\prime}+1} \geq$ $H\left(\alpha^{t^{\prime}+1}\right)+z \geq \alpha^{t^{\prime}}+z \geq r-\epsilon+z=r+\frac{z}{2}$, hence $\alpha^{t^{\prime}+1} \geq r$. This is a contradiction. This completes the proof of step 3.

Step 4. The strategy $\tau$ is obedient.

Proof of Step 4.

Step 4.1: We show that $\tau^{t}\left(h_{r}^{t}, m_{c}\right)=p^{t}=1$ for every period $t$ and history $h_{r}^{t}$ of the receiver. Take any $t \in \mathbb{N}$. It is sufficient to show that $K(t)<U_{r}^{t+1}(\sigma, \tau)$. By step $3, \alpha^{t} \leq \beta$. It holds that

$K(t)=\frac{1}{\alpha^{t}} \cdot \int_{0}^{\alpha^{t}} g^{t}(\theta) d \theta \leq \frac{1}{\beta} \cdot \int_{0}^{\beta} g^{t}(\theta) d \theta<\int_{0}^{1} g^{t+1}(\theta) d \theta \leq U_{r}^{t+1}(\sigma, \tau)$.

In the first inequality, we use Lemma 2.20. In the second inequality, we use Lemma 2.19.3 and the assumption $\delta \cdot V(1)>V(\beta)$. Due to $(\sigma, \tau)$ being a PBE, the last inequality follows because quitting at period $t+1$ cannot be better for the receiver than playing $\tau$ against $\sigma$. This completes the proof of step 4.1 . 
Chapter 2. Incentive compatibility in sender-receiver stopping games

Step 4.2: $\quad$ We show that $\tau^{t}\left(h_{r}^{t}, m_{q}\right)=q^{t}=0$ for every period $t$ and history $h_{r}^{t}$ of the receiver. For every $t \in \mathbb{N}$, let $Q(t)$ be the the statement $U_{r}^{t+1}(\sigma, \tau)<L(t)$. As $(\sigma, \tau)$ is a PBE, $Q(t)$ implies that $q^{t}=0$. It is sufficient to show that $Q(t)$ is true for every $t \in \mathbb{N}$.

Step 4.2.1: We show that $Q(t+1)$ implies $Q(t)$. Suppose that $Q(t+1)$ is true, so $q^{t+1}=0$. Together with step 4.1, it follows that $\tau$ is obedient at period $t+1$. Then

$$
\begin{aligned}
f^{t}\left(\alpha^{t}\right) & =U_{s}^{t+1}(\sigma, \tau) \\
& =\alpha^{t+1} \cdot U_{s}^{t+2}(\sigma, \tau)+\int_{\alpha^{t+1}}^{1} f^{t+1}(\theta) d \theta \\
& =\alpha^{t+1} \cdot f^{t+1}\left(\alpha^{t+1}\right)+\int_{\alpha^{t+1}}^{1} f^{t+1}(\theta) d \theta \\
& =\delta^{t} \cdot G\left(\alpha^{t+1}\right) .
\end{aligned}
$$

The first and third equalities follow from the definitions of $\alpha^{t}$ and $\alpha^{t+1}$ and the last equality from the definition of $G$. The second equality follows from $\tau$ being obedient at period $t+1$.

It follows that $\alpha^{t}=H\left(\alpha^{t+1}\right)$. By step 3, we have $\alpha^{t} \leq \beta$. Hence by Lemma 2.17.3, $\alpha^{t} \geq \alpha^{t+1}$. Then,

$$
\begin{aligned}
U_{r}^{t+1}(\sigma, \tau) & =\alpha^{t+1} \cdot U_{r}^{t+2}(\sigma, \tau)+\int_{\alpha^{t+1}}^{1} g^{t+1}(\theta) d \theta \\
& <\frac{\alpha^{t+1}}{1-\alpha^{t+1}} \cdot \int_{\alpha^{t+1}}^{1} g^{t+1}(\theta) d \theta+\int_{\alpha^{t+1}}^{1} g^{t+1}(\theta) d \theta \\
& =\frac{1}{1-\alpha^{t+1}} \cdot \int_{\alpha^{t+1}}^{1} g^{t+1}(\theta) d \theta \\
& \leq \frac{1}{1-\alpha^{t}} \cdot \int_{\alpha^{t}}^{1} g^{t+1}(\theta) d \theta \\
& <\frac{1}{1-\alpha^{t}} \cdot \int_{\alpha^{t}}^{1} g^{t}(\theta) d \theta \\
& =L(t) .
\end{aligned}
$$

In the first inequality we use that the statement $Q(t+1)$ is true. In the 
second inequality we use Lemma 2.20, which is applicable as $\alpha^{t} \geq \alpha^{t+1}$ by step 3. This completes the proof of step 4.2.1.

Step 4.2.2 We prove that $Q(t)$ is true for every $t$. Assume by way of contradiction that there is a $t \in \mathbb{N}$ for which $Q(t)$ is not true. By step 4.2.1, $Q\left(t^{\prime}\right)$ is not true for all $t^{\prime} \geq t$. Then, by step $2, U_{r}^{t^{\prime}+1}(\sigma, \tau)=$ $L\left(t^{\prime}\right)$ for all $t^{\prime} \geq t$. Denote by $E_{r}^{t+1}$ the expected payoff of the receiver conditional on getting the message $m_{q}$ at period $t+1$. We have

$$
\begin{aligned}
E_{r}^{t+1} & =q^{t+1} \cdot U_{r}^{t+2}(\sigma, \tau)+\left(1-q^{t+1}\right) \cdot L(t+1) \\
& =q^{t+1} \cdot U_{r}^{t+2}(\sigma, \tau)+\left(1-q^{t+1}\right) \cdot U_{r}^{t+2}(\sigma, \tau) \\
& =U_{r}^{t+2}(\sigma, \tau) .
\end{aligned}
$$

The first equality can be explained as follows. On receiving the message $m_{q}$ at period $t+1$, the strategy $\tau$ continues with probability $q^{t+1}$ and on continuing the receiver gets the payoff $U_{r}^{t+2}(\sigma, \tau)$, whereas $\tau$ quits with probability $1-q^{t+1}$ and on quitting the receiver gets the payoff $L(t+1)$. It follows that

$$
U_{r}^{t+1}(\sigma, \tau)=\alpha^{t+1} \cdot U_{r}^{t+2}(\sigma, \tau)+\left(1-\alpha^{t+1}\right) \cdot E_{r}^{t+1}=U_{r}^{t+2}(\sigma, \tau) .
$$

In the first equality we use the step 4.1.

So, $U_{r}^{t+1}(\sigma, \tau)=U_{r}^{t+2}(\sigma, \tau)$. Iterating this argument implies that $U_{r}^{t+1}(\sigma, \tau)=U_{r}^{t+j}(\sigma, \tau)$ for any $j$. As $U_{r}^{t+j} \leq g^{t+j}(1)=\delta^{t+j-1} g(1)$, it then follows that $U_{r}^{t+1}(\sigma, \tau)=0$. However, if the receiver quits at period $t+1$ instead, regardless of the message, then he receives the expected payoff $\int_{0}^{1} g^{t+1}(\theta) d \theta>0$. This contradicts the assumption that $(\sigma, \tau)$ is PBE. This completes the proof of step 4.2.2.

Now Claim 2.15 follows immediately from Proposition 2.3.

Claim 2.16. Suppose that $\delta \in(D, 1)$. Then the regular strategy profile is a PBE. 
Chapter 2. Incentive compatibility in sender-receiver stopping games

Proof. Let $\delta \in(D, 1)$ and let $(\sigma, \tau)$ be the regular strategy profile. From Proposition 2.3, $\sigma$ is stationary with threshold $\beta$. Clearly, $U_{r}^{t+1}(\sigma, \tau)=\delta \cdot U_{r}^{t}(\sigma, \tau)$ for all periods $t \in \mathbb{N}$. We show that $(\sigma, \tau)$ is a PBE.

Since $(\sigma, \tau)$ is regular, we have

$$
U_{r}^{t}(\sigma, \tau)=\beta \cdot U_{r}^{t+1}(\sigma, \tau)+\int_{\beta}^{1} g^{t}(\theta) d \theta=\beta \cdot \delta \cdot U_{r}^{t}(\sigma, \tau)+\int_{\beta}^{1} g^{t}(\theta) d \theta .
$$

It follows that

$$
U_{r}^{t}(\sigma, \tau)=\frac{\delta^{t-1}}{1-\delta \cdot \beta} \cdot \int_{\beta}^{1} g(\theta) d \theta
$$

Now we show that the receiver prefers to play $a_{q}$ on seeing $m_{q}$ at pe$\operatorname{riod} t$. For this, we need to show that

$$
U_{r}^{t+1}(\sigma, \tau) \leq \frac{\delta^{t-1}}{1-\beta} \int_{\beta}^{1} g(\theta) d \theta .
$$

This follows easily from (2.1).

Finally, we show that the receiver prefers to play $a_{c}$ on seeing $m_{c}$ at period $t$. For this, we need to show that

$$
U_{r}^{t+1}(\sigma, \tau) \geq \frac{\delta^{t-1}}{\beta} \int_{0}^{\beta} g(\theta) d \theta .
$$

Using (2.1), the above inequality can be rewritten to

$$
\frac{1}{\beta} \cdot \int_{0}^{\beta} g(\theta) d \theta<\delta \cdot \int_{0}^{1} g(\theta) d \theta .
$$

This follows from Lemma 2.19.3 and the condition $V(\beta)<\delta \cdot V(1)$, which is due to $\delta \in(D, 1)$. 


\subsubsection{The proof of Theorem 2.9}

In this section, we prove Theorem 2.9. By way of contradiction, assume that $(\sigma, \tau)$ is an essentially Markov strategy profile and a responsive PBE. For every period $t$, let $p^{t}=\tau^{t}\left(h_{r}^{t}, m_{c}\right)$ be the probability on the action $a_{c}$ on seeing the message $m_{c}$, and let $q^{t}=\tau^{t}\left(h_{r}^{t}, m_{q}\right)$ be the probability on the action $a_{c}$ on seeing the message $m_{q}$. As $\tau$ is responsive, we know that $p^{t}>q^{t}$.

Step 1. We prove that the sender's strategy $\sigma$ is a threshold strategy with some threshold $\alpha^{t} \in(0,1)$ at each period $t$.

Proof of step 1. Fix a period $t \in \mathbb{N}$. Since $(\sigma, \tau)$ is essentially Markov, $U_{s}^{t+1}(\sigma, \tau)$ is independent of the history up to period $t+1$. The equation $f(x)=U_{s}^{t+1}(\sigma, \tau)$ has a unique solution $\alpha^{t} \in[0,1]$. Notice that $f(0)<$ $U_{s}^{t+1}(\sigma, \tau)<f(1)$ because state 0 are 1 have probability zero. Hence, $\alpha^{t} \in(0,1)$.

Assume first that $\theta^{t}<\alpha^{t}$. Then, $f\left(\theta^{t}\right)<f\left(\alpha^{t}\right)=U_{s}^{t+1}(\sigma, \tau)$. Thus, the sender expects to get a strictly better payoff when the receiver continues than when the receiver quits. As $p^{t}>q^{t}$, the sender strictly prefers to send the message $m_{c}$ over $m_{q}$. Because $(\sigma, \tau)$ is a PBE, $\sigma$ sends the message $m_{c}$ at period $t$.

Assume now that $\theta^{t}>\alpha^{t}$. Then, $f\left(\theta^{t}\right)>f\left(\alpha^{t}\right)=U_{s}^{t+1}(\sigma, \tau)$. By the same reasoning, $\sigma$ sends the message $m_{q}$ at period $t$. This completes the proof of step 1 .

Step 2. We show that for every period $t$, we have $p^{t}=1$.

Suppose that $\sigma$ sends the message $m_{c}$ at period $t$. Then, $\theta^{t} \leq \alpha^{t}$. If the receiver quits at period $t$, his expected payoff is $\int_{0}^{\alpha^{t}} g(\theta) d \theta$. However, if the receiver continues at period $t$ and quits at period $t+1$ irrespective of the message by the sender, his expected payoff is $\int_{0}^{1} g(\theta) d \theta$, which 
Chapter 2. Incentive compatibility in sender-receiver stopping games

is strictly better because $\alpha^{t}<1$ due to step 1 . So, action $a_{q}$ is not a best response for the receiver. It follows that $p^{t}=1$.

Step 3. We show that $\sum_{t=1}^{\infty}\left(1-q^{t}\right)=\infty$.

Let $z^{t}$ denote the probability under $(\sigma, \tau)$ that the receiver continues at period $t$, conditional on reaching period $t$. Thus, $z^{t}=\alpha^{t} \cdot p^{t}+\left(1-\alpha^{t}\right)$. $q^{t}$.

We show that $\Pi_{t=1}^{\infty} z^{t}=0$. Assume by way of contradiction that $\Pi_{t=1}^{\infty} z^{t}>0$. Because

$$
\Pi_{t=1}^{\infty} z^{t}=\Pi_{t=1}^{n} z^{t} \cdot \Pi_{t=n+1}^{\infty} z^{t}
$$

and $\prod_{t=1}^{n} z^{t} \rightarrow \Pi_{t=1}^{\infty} z^{t}$ as $n \rightarrow \infty$, we have $\Pi_{t=n+1}^{\infty} z^{t} \rightarrow 1$ as $n \rightarrow \infty$. So, there is large period $t$ such that conditional on reaching period $t$, the receiver's expected payoff $U_{r}^{t}(\sigma, \tau)$ is less than $\int_{0}^{1} g(\theta) d \theta$. As the receiver can always guarantee an expected payoff of $\int_{0}^{1} g(\theta) d \theta$ by simply quitting, regardless the message sent by the sender, this is a contradiction. Hence, $\Pi_{t=1}^{\infty} z^{t}=0$.

Because $z^{t}=\alpha^{t} \cdot p^{t}+\left(1-\alpha^{t}\right) \cdot q^{t}$, by steps 1 and 2 , we can conclude that $z^{t}>0$. Because $\Pi_{t=1}^{\infty} z^{t}=0$, we obtain $\sum_{t=1}^{\infty}\left(1-z^{t}\right)=\infty$. As $p^{t}>q^{t}$, we also have $z^{t}>q^{t}$. Hence, $\sum_{t=1}^{\infty}\left(1-q^{t}\right)=\infty$.

Step 4. We derive a contradiction.

Let $\epsilon \in(0,1)$. We define a threshold strategy $\sigma_{\epsilon}$ for the sender as follows: at each period $t$, if $\theta^{t}<1-\epsilon$ then $\sigma_{\epsilon}$ sends the message $m_{c}$ and if $\theta^{t} \geq 1-\epsilon$ then $\sigma_{\epsilon}$ sends the message $m_{q}$.

We show that the sender's expected payoff under $\left(\sigma_{\epsilon}, \tau\right)$ is at least $f(1-$ $\epsilon)$. For this it is sufficient to prove that, under $\left(\sigma_{\epsilon}, \tau\right)$, with probability 1 the receiver will eventually quit. Let $z_{\epsilon}^{t}$ denote the probability under $\left(\sigma_{\epsilon}, \tau\right)$ that the receiver continues at period $t$, conditional on reaching 
period $t$. By step $2, z_{\epsilon}^{t}=(1-\epsilon) \cdot p^{t}+\epsilon \cdot q^{t}=(1-\epsilon)+\epsilon \cdot q^{t}$. Thus, by step $3, \sum_{t=1}^{\infty}\left(1-z_{\epsilon}^{t}\right)=\epsilon \cdot \sum_{t=1}^{\infty}\left(1-q^{t}\right)=\infty$. As $z_{\epsilon}^{t}>0$ for each $t$, we obtain $\Pi_{t=1}^{\infty} z_{\epsilon}^{t}=0$. Thus, under $\left(\sigma_{\epsilon}, \tau\right)$, with probability 1 the receiver will eventually quit.

Since the sender can guarantee, for every $\epsilon \in(0,1)$, an expected payoff of at least $f(1-\epsilon)$ against $\tau$, the sender's expected payoff under $(\sigma, \tau)$ must be at least $f(1)$. Since the state 1 is realized with probability 0 , this is a contradiction. This concludes the proof.

\section{Appendix}

\section{A. The receiver's belief on the history of the sender}

In this appendix, we describe the receiver's conditional probability distribution (or belief) $\mathbb{P}_{\sigma, \tau, h_{r}^{t}}$ on the set $H_{s}^{t}$ of possible histories for the sender, given the strategy profile $(\sigma, \tau)$ and the receiver's history $h_{r}^{t}=\left(m^{1}, m^{2}, \ldots, m^{t-1}\right)$.

Let $\sigma^{k}\left(m^{k} \mid h_{s}^{k}, \theta^{k}\right)$ denote the probability on the message $m^{k}$ under the strategy $\sigma$, given the history $h_{s}^{k}$ and the state $\theta^{k}$. For numbers $y^{1}, y^{2}, \ldots, y^{t-1} \in[0,1]$, the expression

$$
\int_{0}^{y^{1}} \int_{0}^{y^{2}} \ldots \int_{0}^{y^{t-1}}\left[\prod_{k=1}^{t-1} \sigma^{k}\left(m^{k} \mid \theta^{1}, m^{1}, \ldots, \theta^{k-1}, m^{k-1}, \theta^{k}\right)\right] d \theta^{t-1} \ldots d \theta^{1}
$$

is the probability of the event that $\theta^{1} \leq y^{1}, \theta^{2} \leq y^{2}, \ldots, \theta^{t-1} \leq y^{t-1}$ and the messages sent are $m^{1}, m^{2}, \ldots, m^{t-1}$. We denote this probability by $\chi_{(\sigma, \tau)}^{t}\left(h_{r}^{t}\right)\left(y^{1}, y^{2}, \ldots, y^{t-1}\right)$.

The quantity $\chi_{(\sigma, \tau)}^{t}\left(h_{r}^{t}\right)(1,1, \ldots, 1)$ is the probability that the history at period $t$ is $h_{r}^{t}$. Thus, the probability of the event that $\theta^{1} \leq y^{1}, \theta^{2} \leq y^{2}$, 
Chapter 2. Incentive compatibility in sender-receiver stopping games

$\ldots, \theta^{t-1} \leq y^{t-1}$ conditional on the messages $m^{1}, m^{2}, \ldots, m^{t-1}$ is

$$
\Psi_{(\sigma, \tau)}^{t}\left(h_{r}^{t}\right)\left(y^{1}, y^{2}, \ldots, y^{t-1}\right)=\frac{\chi_{(\sigma, \tau)}^{t}\left(h_{r}^{t}\right)\left(y^{1}, y^{2}, \ldots, y^{t-1}\right)}{\chi_{(\sigma, \tau)}^{t}\left(h_{r}^{t}\right)(1,1, \ldots, 1)} .
$$

If a certain history $h_{r}^{t}$ occurs with probability zero, that is, if $\chi_{(\sigma, \tau)}^{t}\left(h_{r}^{t}\right)(1,1, \ldots, 1)=0$, then we define $\Psi_{(\sigma, \tau)}^{t}\left(h_{r}^{t}\right)$ to be any probability distribution. The choice of this probability distribution plays no role in our proofs. The probabilities $\Psi_{(\sigma, \tau)}^{t}\left(h_{r}^{t}\right)\left(y^{1}, y^{2}, \ldots, y^{t-1}\right)$ induce the desired probability measure $\mathbb{P}_{\sigma, \tau, h_{r}^{t}}$ on the possible histories $h_{s}^{t}$ for the sender.

\section{B. Expected payoff}

In this appendix, we provide the details of how the expected payoffs $U_{s}(\sigma, \tau), U_{r}(\sigma, \tau)$ and the continuation expected payoffs $U_{s}^{t}(\sigma, \tau)\left(h_{s}^{t}\right)$ and $U_{r}^{t}(\sigma, \tau)\left(h_{r}^{t}\right)$ from period $t$ onward can be calculated.

It is both convenient and standard to assume that even if the receiver quits at some period $t$, play continues indefinitely, but actions in any period beyond $t$ have no influence on the payoffs. With this assumption, a play of the game is a sequence $\omega=\left(\theta^{t}, m^{t}, a^{t}\right)_{t=1}^{\infty}$ where $\theta^{t} \in I$, $m^{t} \in M$ and $a^{t} \in A$. Denote by $\Omega=(I \times M \times A)^{\mathbb{N}}$ the set of all plays. Given the usual Borel sigma-algebra of $I$, we endow $\Omega$ with the product sigma-algebra $\mathcal{B}$.

With abuse of notation, define $\theta^{t}: \Omega \rightarrow I, m^{t}: \Omega \rightarrow M$ and $a^{t}: \Omega \rightarrow A$ to be the projection maps from the set of plays, respectively to the state, the message and the action at period $t$. Let $S: \Omega \rightarrow \mathbb{N} \cup\{\infty\}$ be the mapping such that, for each $\omega \in \Omega, S(\omega)$ is the first period $t$ for which $a^{t}(\omega)=a_{q}$. If there is no such $t$ then $S(\omega)=\infty$. It is the stopping time which indicates when the game effectively ends. For a play $\omega$, the 
payoffs for the players are given as follows

$$
\begin{aligned}
& \Pi_{s}(\omega)=f^{S(\omega)}\left(\theta^{S(\omega)}(\omega)\right) \cdot \mathbb{1}_{\{S(\omega)<\infty\}}, \\
& \Pi_{r}(\omega)=g^{S(\omega)}\left(\theta^{S(\omega)}(\omega)\right) \cdot \mathbb{1}_{\{S(\omega)<\infty\}} .
\end{aligned}
$$

Any fixed strategy profile $(\sigma, \tau)$ induces a probability measure on the measurable space $(\Omega, \mathcal{B})$, denoted by $\mathbb{P}_{\sigma, \tau}$. The expectation with respect to this probability measure is denoted by $\mathbb{E}_{\sigma, \tau}$. The expected payoff for the sender is given by $U_{s}(\sigma, \tau)=\mathbb{E}_{\sigma, \tau}\left[\Pi_{s}(\omega)\right]$ and the expected payoff for the receiver is given by $U_{r}(\sigma, \tau)=\mathbb{E}_{\sigma, \tau}\left[\Pi_{r}(\omega)\right]$.

Let $\Omega^{\geq t}$ denote the set of all continuation plays $\omega^{\geq t}=\left(\theta^{k}, m^{k}, a^{k}\right)_{k=t}^{\infty}$. Given a history $h_{s}^{t} \in H_{s}^{t}$ for the sender, the continuation strategy $\sigma\left[h_{s}^{t}\right]=\left(\sigma^{k}\left[h_{s}^{t}\right]\right)_{k=1}^{\infty}$ of $\sigma$ is defined in the usual way: for each period $k \in \mathbb{N}$, history $\bar{h}_{s}^{k} \in H_{s}^{k}$ and state $\theta^{k} \in I$ we let

$$
\sigma^{k}\left[h_{s}^{t}\right]\left(h_{s}^{k}, \theta^{k}\right)=\sigma^{t+k-1}\left(h_{s}^{t}, h_{s}^{k}, \theta^{k}\right) .
$$

Given a history $h_{r}^{t} \in H_{r}^{t}$ for the receiver, we define in a similar way the continuation strategy $\tau\left[h_{r}^{t}\right]=\left(\tau^{k}\left[h_{r}^{t}\right]\right)_{k=1}^{\infty}$ of $\tau$.

For each period $t$, let $\pi^{t}: H_{s}^{t} \rightarrow H_{r}^{t}$ be the map that projects the sender's history to the receiver's history. For a given history $h_{s}^{t}$ of the sender, the continuation strategies $\sigma\left[h_{s}^{t}\right]$ and $\tau\left[\pi\left(h_{s}^{t}\right)\right]$ induce a probability measure on the space $(\Omega, \mathcal{B})$, denoted by $\mathbb{P}_{\sigma, \tau, h_{s}^{t}}$. The expected continuation payoff for the sender is given by $U_{s}^{t}(\sigma, \tau)\left(h_{s}^{t}\right)=\mathbb{E}_{\sigma, \tau, h_{s}^{t}}\left[\Pi_{s}(\omega)\right]$.

As discussed in Appendix A, the receiver has a probability distribution (belief) $\mathbb{P}_{\sigma, \tau, h_{r}^{t}}$ on the set $H_{s}^{t}$, conditional on his history $h_{r}^{t}$. The expected continuation payoff for the receiver is can be calculated as follows

$$
U_{r}^{t}(\sigma, \tau)\left(h_{r}^{t}\right)=\int_{H_{s}^{t}} U_{r}\left(\sigma\left[h_{s}^{t}\right], \tau\left[h_{r}^{t}\right]\right) \mathbb{P}_{\sigma, \tau, h_{r}^{t}}\left(d h_{s}^{t}\right) .
$$


Chapter 2. Incentive compatibility in sender-receiver stopping games

Here, the integrand $U_{r}\left(\sigma\left[h_{s}^{t}\right], \tau\left[h_{r}^{t}\right]\right)$ is the receiver's expected payoff given the continuation strategies $\sigma\left[h_{s}^{t}\right]$ and $\tau\left[h_{r}^{t}\right]$.

\section{Extension: Arbitrary distribution}

We consider an extension in which the states at each period are drawn from an arbitrary distribution for the games with finite or infinite horizon and with payoffs that are discounted or period independent.

Consider a sender-receiver game where the payoffs are either discounted $(\delta<1)$ or period independent $(\delta=1)$. Let the characteristic functions $f$ and $g$ from $I$ to $\mathbb{R}_{+}$be strictly increasing with $f(0)=g(0)=0$. At each period $t$, the state $\theta^{t}$ is drawn from a fixed cumulative distribution $F$ on $[0,1]$, independently from realized states of previous periods. We assume that $F$ is strictly increasing and continuous on $[0,1]$ and $F(0)=0$. We denote this game by $\mathcal{G}^{F}$.

Using the game $\mathcal{G}^{F}$, we define a new game $\mathcal{G}^{u}$ with the same horizon $T$ in which the states at each period $t$ in the game $\mathcal{G}^{u}$ are drawn from the uniform distribution independently from states of previous periods. The game $\mathcal{G}^{u}$ has the same $\delta$ as the game $\mathcal{G}^{F}$ and has the characteristic functions $\hat{f}$ and $\hat{g}$ which are defined as follows: $\hat{f}(x)=f\left(F^{-1}(x)\right)$, $\hat{g}(x)=g\left(F^{-1}(x)\right)$.

Given a strategy profile $(\sigma, \tau)$ in the game $\mathcal{G}^{F}$, consider a strategy profile $(\hat{\sigma}, \hat{\tau})$ in the game $\mathcal{G}^{u}$, defined as follows: $\hat{\sigma}^{t}\left(\theta^{t}\right)=\sigma^{t}\left(F^{-1}\left(\theta^{t}\right)\right)$ and $\hat{\tau}^{t}\left(m^{t}\right)=\tau^{t}\left(m^{t}\right)$. It is straightforward, but tedious to show that the payoffs of the players in the game $\mathcal{G}^{u}$ when the strategy profile is $(\hat{\sigma}, \hat{\tau})$ and in the game $\mathcal{G}^{F}$ when the strategy profile is $(\sigma, \tau)$ are exactly same.

Under this transformation, the receiver's strategy remains the same. If the sender's strategy $\sigma$ in $\mathcal{G}^{F}$ is a threshold strategy with a threshold $\alpha^{t}$ at period $t$, then $\hat{\sigma}$ is also a threshold strategy with threshold $F^{-1}\left(\alpha^{t}\right)$ at period $t$. So, the regular strategy profile in $\mathcal{G}^{F}$ is transformed into the regular strategy profile in $\mathcal{G}^{u}$. Hence, the existence and unicity results 
in the game $\mathcal{G}^{u}$ can be used to derive the existence and unicity results in the game $\mathcal{G}^{F}$.

\section{Auxiliary lemmas}

For the statement and proofs in the appendix, we fix the strictly increasing continuous functions $f$ and $g$ from $I$ to $\mathbb{R}_{+}$such that $f(0)=0$, $g(0)=0$.

Consider an auxiliary function $G: I \rightarrow \mathbb{R}$ is defined as $G(x)=x$. $f(x)+\int_{x}^{1} f(\theta) d \theta$ and recall the function $H: I \rightarrow \mathbb{R}$ defined as $H(x)=$ $f^{-1}(\delta \cdot G(x))$.

Lemma 2.17. The following statements hold:

[1] The functions $G, H$ are strictly increasing,

[2] The function $H$ has a unique fixed point, denoted by $\beta$,

[3] $H(y)>y$ for all $y<\beta$ and $H(y)<y$ for all $y>\beta$,

[4] $\beta \rightarrow 1$ as $\delta \rightarrow 1$ and $\beta=1$ when $\delta=1$.

Proof. [1] Take $0 \leq x<y \leq 1$. Because $f(x)<f(y)$, we have

$$
\begin{aligned}
G(y)-G(x) & =\left[y f(y)+\int_{y}^{1} f(\theta) d \theta\right]-\left[x f(x)+\int_{x}^{1} f(\theta) d \theta\right] \\
& =y f(y)-x f(x)-\int_{x}^{y} f(\theta) d \theta \\
& \geq(y-x) f(y)-\int_{x}^{y} f(\theta) d \theta>0 .
\end{aligned}
$$

Hence, $G$ is strictly increasing. The monotonicity of $H$ follows easily.

[2] We have $H(0)>0$ and $H(1) \leq 1$. As $H$ is strictly increasing, there exists $x \in I$ such that $H(x)=x$. Let $\beta=\inf \{x \in I \mid H(x)=x\}$. By 
Chapter 2. Incentive compatibility in sender-receiver stopping games

continuity of $H$, we have $H(\beta)=\beta$. Now we will prove part 3 of the lemma. This will imply that $\beta$ is the unique solution of $H(x)=x$.

[3] We will show that the function $k(x)=f(H(x))-f(x)$ is decreasing in $x \in I$. So, take $0 \leq x<y \leq 1$. We have

$$
\begin{aligned}
k(y)-k(x) & =[\delta \cdot G(y)-f(y)]-[\delta \cdot G(x)-f(x)] \\
& =\delta \cdot[G(y)-G(x)]-f(y)+f(x) \\
& =\delta \cdot\left[y f(y)-x f(x)-\int_{x}^{y} f(\theta) d \theta\right]-f(y)+f(x) \\
& =-(1-\delta y) \cdot f(y)+(1-\delta x) \cdot f(x)-\delta \cdot \int_{x}^{y} f(\theta) d \theta \\
& \leq-(1-\delta y) \cdot f(x)+(1-\delta x) \cdot f(x)-\delta \cdot \int_{x}^{y} f(\theta) d \theta \\
& =\delta \cdot\left[(y-x) \cdot f(x)-\int_{x}^{y} f(\theta) d \theta\right]<0 .
\end{aligned}
$$

So the function $k(x)=f(H(x))-f(x)$ is indeed decreasing in $x \in I$.

Notice that $k(\beta)=0$. Assume that $y<\beta$. Then, since $k$ is decreasing, we have $k(y)>k(\beta)=0$. Hence, $f(H(y))>f(y)$, so $H(y)>y$. Similarly, if $y>\beta$ then $H(y)<y$.

[4] As $\beta$ is the unique fixed point of $H$, and for $\delta=1$ we have $H(1)=1$, it follows that $\beta=1$ when $\delta=1$. A continuity argument shows that $\beta \rightarrow 1$ as $\delta \rightarrow 1$. This completes the proof.

For the next lemma, let the game has the finite horizon $T$. Recall that $\beta^{T}=0$ and $\beta^{t}=H\left(\beta^{t+1}\right)$ for all $t=1, \ldots, T-1$ and the function $V$ is defined as $V(x)=\frac{1}{x} \cdot \int_{0}^{x} g(\theta) d \theta$. Whenever necessary we use the notation $\beta^{t}(T)$ for the threshold at period $t$ to specify the horizon $T$.

Lemma 2.18. The following statements hold:

[1] $1 \geq \beta>\beta^{1}>\beta^{2}>\cdots>\beta^{T}=0$. 
[2] $\frac{1}{\beta^{t}} \cdot \int_{0}^{\beta^{t}} g^{t}(\theta) d \theta<\int_{0}^{1} g^{t+1}(\theta) d \theta$ for $\delta \in\left[D^{T}, 1\right]$ and $t=1, \ldots, T-1$

[3] $\beta^{1}(T) \rightarrow \beta$ as $T \rightarrow \infty$. More generally, $\beta^{t}(T) \rightarrow \beta$ as $T \rightarrow \infty$ for each $t$.

Proof. [1] By definition, $\beta^{T}=0$. We also have

$$
\beta^{T-1}=H\left(\beta^{T}\right)=H(0)=f^{-1}\left(\delta \cdot \int_{0}^{1} f(\theta) d \theta\right)>0 .
$$

So, $\beta^{T-1}>\beta^{T}$. Then inductively $\beta^{t}=H\left(\beta^{t+1}\right)>H\left(\beta^{t+2}\right)=\beta^{t+1}$ for all $t=T-2, \ldots, 1$. Thus, $\beta^{1}>\beta^{2}>\cdots>\beta^{T}=0$.

As $\beta^{1}>\beta^{2}$, we have $H\left(\beta^{1}\right)>H\left(\beta^{2}\right)=\beta^{1}$. So, by lemma 2.17.3, we have $\beta^{1}<\beta$.

Finally, $\beta=H(\beta) \leq f^{-1}(\delta f(1))<f^{-1}(f(1))=1$, so $\beta<1$. This completes the proof.

[2] It holds that

$$
\frac{1}{\beta^{t}} \cdot \int_{0}^{\beta^{t}} g^{t}(\theta) d \theta<\frac{1}{\beta^{1}} \cdot \int_{0}^{\beta^{1}} g^{t}(\theta) d \theta \leq \delta \cdot \int_{0}^{1} g^{t}(\theta) d \theta=\int_{0}^{1} g^{t+1}(\theta) d \theta .
$$

The first inequality follows from from substituting $a=b=0, c=\beta^{t}$, and $d=\beta^{1}$ into Lemma 2.20. The second inequality follows from the fact that $\delta \cdot V(1) \geq V\left(\beta^{1}\right)$, which is true due to the assumption $\delta \in$ $\left[D^{T}, 1\right]$.

[3] By definition, we have $\beta^{t}(T)=\beta^{1}(T-t+1)$ for $t \leq T$. So, it is sufficient to show that $\beta^{1}(T) \rightarrow \beta$ as $T \rightarrow \infty$. By part [1], we have $\beta^{t}(T)>\beta^{t+1}(T)$. So, we have $\beta^{1}(T-t+1)>\beta^{1}(T-t)$ for $t<T$. By replacing $T-t+1$ to $T$, we obtain $\beta^{1}(T)>\beta^{1}(T-1)$ for any $T>1$.

As $H$ is strictly increasing, we have $H\left(\beta^{1}(T)\right)>H\left(\beta^{1}(T-1)\right)=\beta^{1}(T)$. So, by lemma 2.17.3, $\beta^{1}(T)<\beta$ for all $T \in \mathbb{N}$. Hence the sequence $\left(\beta^{1}(T)\right)_{T \in \mathbb{N}}$ is strictly increasing and bounded above by $\beta$. Assume that the sequence converges to $y \in[0, \beta]$. We need to show that $y=\beta$. 
Chapter 2. Incentive compatibility in sender-receiver stopping games

We now will assume that $y<\beta$ and show a contradiction, which will prove $y=\beta$. Denote $z=\min \{H(x)-x: x \in[0, y]\}$. By Lemma 2.17.3, $H(x)>x$ for $x \leq y<\beta$. By continuity of $H$, we have $z>0$.

As the sequence $\left(\beta^{1}(T)\right)_{T \in \mathbb{N}}$ converges to $y$, for $\epsilon=\frac{z}{2}$, there exists $T^{\prime}>$ 0 such that $\beta^{1}(T) \in[y-\epsilon, y]$ for all $T>T^{\prime}$. Now for any fixed $T>T^{\prime}$, consider $\beta^{1}(T+1)=H\left(\beta^{1}(T)\right) \geq \beta^{1}(T)+z \geq y-\epsilon+z=y+\frac{z}{2}$. This is a contradiction as $T+1>T^{\prime}$. This completes the proof.

Recall that the function $f: I \rightarrow \mathbb{R}$ is Lipschitz at 1 if there exist a constant $M>0$ and number $Y \in(0,1)$ such that $f(1)-f(y) \leq M$. $(1-y)$ for all $y \in[Y, 1]$.

Lemma 2.19. The following statements hold:

[1] If $f$ is Lipschitz at 1, then for every $K>0$ there is $E \in(0,1)$ such that for all $\delta \in[E, 1]$,

$$
1-\beta \geq K \cdot(1-\delta)
$$

[2] If $f$ is Lipschitz at 1, then there is $D \in[0,1)$ such that for all $\delta \in[D, 1]$,

$$
\delta \cdot V(1) \geq V(\beta) .
$$

[3] $\frac{1}{\beta} \cdot \int_{0}^{\beta} g^{t}(\theta) d \theta<\int_{0}^{1} g^{t+1}(\theta) d \theta$ for $\delta \in(D, 1)$.

Proof. [1] The proof of part 1 is in two parts.

A. Write $k(\delta)=1-\delta \cdot \beta$. We first show that

$$
(1-\delta) \cdot f(1) \leq[f(1)-f(\beta)] \cdot k(\delta) .
$$

Note that, by definition of $\beta$,

$$
f(\beta)=\delta \cdot G(\beta)=\delta \cdot\left[\beta \cdot f(\beta)+\int_{\beta}^{1} f(\theta) d \theta\right] .
$$


This yields

$$
f(\beta)[1-\delta \cdot \beta]=\delta \cdot \int_{\beta}^{1} f(\theta) d \theta \leq \delta \cdot(1-\beta) \cdot f(1) .
$$

Expanding brackets and adding $f(1)$ to both sides yields the inequality

$$
f(1)+f(\beta)-\delta \cdot \beta \cdot f(\beta) \leq f(1)+\delta f(1)-\delta \cdot \beta \cdot f(1) .
$$

This can be rewritten into

$$
(1-\delta) \cdot f(1) \leq[f(1)-f(\beta] \cdot k(\delta) .
$$

B. We continue with the proof of the statement. Since the function $f: I \rightarrow \mathbb{R}$ is Lipschitz at 1 , there exist a constant $M>0$ and number $Y \in(0,1)$ such that $f(1)-f(y) \leq M \cdot(1-y)$ for all $y \in[Y, 1]$.

Take $K>0$. Define $L=\frac{f(1)}{K \cdot M}$. As $\delta$ tends to $1, k(\delta)=1-\delta \cdot \beta$ tends to 0 . So, there is $E \in[Y, 1)$ such that $k(\delta) \leq L$ for all $\delta \in[E, 1]$. Take any $\delta \in[E, 1]$.

If $\delta=1$, then inequality (2.2) is trivially true. So suppose that $\delta \in[E, 1)$. Then, using the result from $\mathrm{A}$,

$$
\begin{aligned}
(1-\delta) \cdot f(1) & \leq \frac{f(1)-f(\beta)}{1-\beta} \cdot(1-\beta) \cdot k(\delta) \\
& \leq M \cdot(1-\beta) \cdot k(\delta) \\
& \leq M \cdot(1-\beta) \cdot L \\
& =(1-\beta) \cdot \frac{f(1)}{K} .
\end{aligned}
$$

Since $f(1)>0$, the part 1 of the lemma follows.

[2] Define

$$
k(x)=g(x)-\frac{1}{x} \int_{0}^{x} g(\theta) d \theta
$$

for all $x \in(0,1]$. Note that $k$ is continuous and $k(1)>0$. Hence, there are $\varepsilon>0$ and $C \in(0,1)$ with $k(x) \geq \varepsilon$ for all $x \geq C$. Define $K=\frac{V(1)}{\varepsilon}$. 
Chapter 2. Incentive compatibility in sender-receiver stopping games

Take $E \in(0,1)$ as in Lemma 2.19.1. In view of Lemma 2.17.4, there is $F \in(0,1)$ such that $\beta \geq C$ for all $\delta \in[F, 1]$. Take $D=\max \{E, F\}$. Take any $\delta \in[D, 1]$. Then

$$
\begin{aligned}
V(1)-V(\beta) & =\int_{0}^{1} g(\theta) d \theta-\frac{1}{\beta} \int_{0}^{\beta} g(\theta) d \theta \\
& \geq(1-\beta) \cdot g(\beta)+\int_{0}^{\beta} g(\theta) d \theta-\frac{1}{\beta} \int_{0}^{\beta} g(\theta) d \theta \\
& =(1-\beta) \cdot\left[g(\beta)-\frac{1}{\beta} \int_{0}^{\beta} g(\theta) d \theta\right] \\
& =(1-\beta) \cdot k(\beta) \\
& \geq(1-\beta) \cdot \varepsilon \\
\geq & K \cdot(1-\delta) \cdot \varepsilon \\
& =(1-\delta) \cdot V(1) .
\end{aligned}
$$

It follows that $\delta \cdot V(1) \geq V(\beta)$ for all $\delta \in[D, 1]$.

[3] It holds that

$$
\frac{1}{\beta} \cdot \int_{0}^{\beta} g^{t}(\theta) d \theta<\delta \cdot \int_{0}^{1} g^{t}(\theta) d \theta=\int_{0}^{1} g^{t+1}(\theta) d \theta .
$$

The first inequality follows the fact that $\delta \cdot V(1) \geq V(\beta)$, which is true due to the assumption $\delta \in(D, 1)$.

Lemma 2.20. Let $g: \mathbb{R} \rightarrow \mathbb{R}$ be a non-decreasing function. Then, for any $a \leq b<c \leq d$,

$$
\frac{1}{c-a} \int_{a}^{c} g(x) d x \leq \frac{1}{d-b} \int_{b}^{d} g(x) d x .
$$

The inequality is strict if $g$ is strictly increasing.

Proof. Define $\eta:[a, c] \rightarrow[b, d]$ by

$$
\eta(x)=\frac{d-b}{c-a} \cdot x+\frac{b c-a d}{c-a} .
$$


Then $\eta(a)=b$ and $\eta(c)=d$, and $\eta$ is a linear bijection between $[a, c]$ and $[b, d]$. Because $a \leq b$ and $c \leq d$ and $\eta$ is linear, we have $x \leq \eta(x)$ for all $x \in[a, c]$.

Write $h(x)=(g \circ \eta)(x)$ for all $x \in[a, c]$. Since $x \leq \eta(x)$ and $g$ is nondecreasing, $g \leq h$ on $[a, c]$. By using substitution, it follows that

$$
\begin{aligned}
\frac{1}{c-a} \int_{a}^{c} g(x) d x & \leq \frac{1}{c-a} \int_{a}^{c} h(x) d x \\
& =\frac{1}{d-b} \int_{a}^{c}(g \circ \eta)(x) d \eta(x) \\
& =\frac{1}{d-b} \int_{b}^{d} g(y) d y .
\end{aligned}
$$

This completes the proof. 



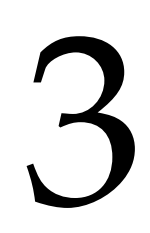

\section{Sender-Receiver stopping games with multiple senders}




\subsection{Introduction}

As discussed in Chapter 2, since Crawford and Sobel [9], there has been significant amount of work in the theory of sender-receiver games. In their settings, a better informed sender is in the position to transmit information to a lesser informed or even uninformed receiver. The key focus in these papers is the characterization of the set of equilibrium payoffs where, in the spirit of the folk theorem, the players are sufficiently patient. Recently there has been work on the extensions of the sender-receiver setting in which there are multiple senders.

In Chapter 2, we introduced the model of sender-receiver stopping games, which combines features from dynamic sender-receiver games and stopping games. In these games the strategic information transmission takes place repeatedly until the receiver decides to stop the interaction. Their main result is the characterization of Perfect Bayesian Equilibrium (PBE) in which the receiver's strategy is non-babbling. The main feature of this chapter is the regular strategy profile which consists of a threshold strategy for the sender and the strategy for the receiver which always follows the recommendation of the sender.

In this chapter ${ }^{1}$ we consider the setting of sender-receiver stopping games with more than one sender and only one receiver. In these games the senders and the receiver interact repeatedly until the receiver decides to stop the interaction. We focus on finite horizon, that is, the receiver is forced to stop the game before a pre-defined terminal period has been reached. The timing of the game is as follows: In each period nature draws a state of the world, which is revealed to all the senders but not to the receiver. After observing the state of the world, each sender sends one out of two messages to the receiver. This message is interpreted as a suggestion either to stop the game or to continue. The receiver has to take a decision, after seeing the messages from all the senders, but without knowing the state. The receiver

\footnotetext{
${ }^{1}$ This chapter is based on joint work with János Flesch, Mathias Staudigl and Dries Vermeulen. I would like to thank Arunava Sen for the discussion and the suggestions on the extension of the work in Chapter 2.
} 
has two options: he can decide to stop the game, or he can decide to continue to the next period. The payoff to each player is a function of the state at which the receiver stops, and these payoffs are either discounted or undiscounted.

We assume that rewards are positively correlated with the state of nature, i.e. higher states lead to better payoffs for all the players. Thus, all the players have identical ordinal preferences over realizations of the state of nature. Yet, as we impose no further restrictions on the payoffs, the cardinal assignment of values of the payoff functions can be very different. As a consequence, a certain state may be very appealing to one player, but not so much to the other, creating an strategic tension between the parties. In particular, the senders have different preferences over whether should receiver should stop the game, or continue to the next period.

This chapter investigates to what extent the cardinal differences between the payoffs may hamper coordination between the different senders or the a sender and the receiver. The main solution concept that we use to analyze these games is Perfect Bayesian Equilibrium (PBE). We identify a class of intuitive PBEs which are appealing to the players in terms of payoffs, and moreover easy to compute and implement.

\subsubsection{Our contribution}

We are interested in studying the PBEs in these games. When there is only 1 sender and 1 receiver, the receiver relies only on the messages of that sender. Hence the sender is able to manipulate the receiver's lack of information for achieving his desired outcome. In fact [1] shows that when players are sufficiently patient, the unique PBE where the receiver's strategy is not babbling, is such that the receiver obediently obeys the recommendations of the sender. However, when there are more than one senders, the receiver has different sources of information about the state. Hence, the crucial question is whether the receiver 
benefits from having more than one senders to make better informed decisions, whether the senders still have manipulative power over the receiver in order to get desired outcomes.

Some of these concerns are captured by the regular strategy profile. In the regular strategy profile, each sender sends a sincere message, given the realization of the state, whether or not he would like the receiver to terminate the game at this period. The sender's sincere strategy is a threshold strategy that sends the message "continue" if the current state is below the threshold and sends the message "quit" otherwise. In the regular strategy profile, the receiver plays the reactive strategy. The reactive strategy is a threshold strategy that quits if the number of senders who send the message quit is more than or equal to the threshold and continues if the number of senders who send the message quit is less than the threshold.

The choice of the thresholds for the reactive strategy has a very intuitive interpretation. Assuming that each sender is playing the sincere strategy, the thresholds of the senders are known ${ }^{2}$. Depending on the realized state, the senders with lower thresholds will send the message quit, and the senders with higher thresholds will send the message continue. So the thresholds of the senders divide the state space into smaller continuous intervals. Given a set of messages, the receiver concludes which interval the state is in, thus is better informed. He prefers to continue if the state is in lower intervals and to quit if the state is in higher intervals. Hence, the choice of the threshold corresponds to the lowest interval where the receiver prefers to quit.

We show that in the finite horizon game, the regular strategy profile is a PBE in which the receiver's strategy is not babbling. Moreover, in the regular strategy profile, no coalition of senders can deviate so that each sender in the coalition gets strictly higher expected payoff. However, the receiver can collude with some senders such that all of them are

\footnotetext{
${ }^{2}$ Although the reactive strategy is constructed taking into the consideration the thresholds of the senders and therefore their payoff functions, we do not need the assumption that the receiver knows the senders' payoff functions.
} 
better off. Unlike in the setting of one sender and one receiver, the regular strategy profile is not unique. In fact we show some interesting examples of PBEs which are not regular strategy profiles. In one such example, the receiver receives the best possible expected payoff among all the strategy profiles, not necessarily PBEs.

We also study how does having an additional sender affect the payoffs of the receiver as well as the other senders, while fixing the strategy profile to the regular strategy profile. Having an additional sender implies more information for the receiver. So intuitively the receiver should have higher expected payoff and the remaining senders should have lower expected payoffs. However we show with the examples that in general this is not true.

\subsubsection{Related literature}

Crawford and Sobel [9] introduced a model of strategic information transmission. The model in which the sender and the receiver interact only once is studied extensively, see $[9,18]$. Recently a lot of work has examined dynamic extensions, where the strategic interaction takes place repeatedly either for finite or infinite number of periods, see [31, 17, 26, 3]. [31] assume that the sequence of states follows an irreducible Markov chain. They characterize the limit set of equilibrium payoffs, as players become very patient. [17] study finite horizon games, and show that, under certain conditions, full information revelation is possible and conditioning future information release on past actions improves incentives for information revelation.

This chapter also relates to the large and growing literature of Bayesian persuasion, see $[21,12,32,19]$. In these settings, the informed advisor (i.e. the sender) decides how much information to share with a less informed agent (i.e. the receiver) so as to influence his decision. [32] show that in many cases, the optimal greedy disclosure policy for the sender exists, which at each stage, minimizes the amount of informa- 
tion being disclosed in that stage under the constraint that it maximizes the current payoff of the sender.

This chapter also relates to the classical contributions on communication in games (see e.g. [14, 29]). In [36, 6], the evolution of information flow is studied in the setting of strategic communication. Our model is also linked to topics in computer science such as automated advice provision. For instance, [4] use sender-receiver games to model interaction between computers and humans.

This chapter relates to work on multi-sender communication (e.g., [5], [24], [28]). Broadly, our work relates to competition between the information providers (see e.g. [22], [15], [2], [23]).

Most of the previous work has focused on the sender-receiver games with multiple senders with fixed duration of time. Compared to these earlier papers, one of the main unique features of our model is that the game is a stopping game. The receiver has the license to stop the game at any period of time. In this respect, our model is connected to the literature on stopping games, see for example [37,11], or even to the secretary problem, see [13].

The structure of this chapter is as follows. In section 3.2 we introduce the model and in the section 3.3, we discuss the regular strategy profile. In section 3.4 we state our main result about the regular strategy being a PBE. In section 3.5, we give examples that illustrate our main results and some examples that show other interesting PBEs. In section 3.6 we examine the externalities of having an additional sender. Section 3.7 concludes.

\subsection{The model}

In this section we describe the model of sender-receiver stopping games with multiple senders and the solution concept of Perfect Bayesian Equilibrium. 


\subsubsection{The game}

We study sender-receiver stopping games with multiple senders and finite horizon. These are dynamic games played by the set of senders $N=\{1, \ldots, n\}$, where $n \geq 1$, and the receiver on a finite horizon $T$, where $T \geq 2$.

A sender-receiver stopping game with multiple senders and finite horizon $T$ is played at periods $t=1,2, \ldots T$. At a period $t$, play is as follows. First, a state of the world $\theta^{t}$ is drawn uniformly from the unit interval $I=[0,1]$, independently of $\theta^{1}, \ldots, \theta^{t-1}$. Each sender learns $\theta^{t}$, while the receiver only knows the distribution of $\theta^{t}$. Next, each sender $i \in N$ chooses a message $m_{i}^{t} \in\left\{m_{c}, m_{q}\right\}$ and sends it to the receiver. The message $m_{i}^{t}=m_{c}$ is interpreted as a suggestion from $i$ for the receiver to continue at this period $t$ and the message $m_{i}^{t}=m_{q}$ as a suggestion to quit. After observing the message profile $m^{t}=\left(m_{1}^{t}, m_{2}^{t}, \ldots, m_{n}^{t}\right)$, the receiver chooses an action $a^{t} \in\left\{a_{c}, a_{q}\right\}$, where $a_{c}$ stands for continue and $a_{q}$ stands for quit. If the receiver quits then the game ends at period $t$, whereas if the receiver continues then the game proceeds to period $t+1$. If the game ends at period $t$, then the sender $i$ receives the payoff $\delta^{t-1} \cdot f_{i}\left(\theta^{t}\right)$ and the receiver receives the payoff $\delta^{t-1} \cdot g\left(\theta^{t}\right)$. Here, $f_{i}$ and $g$ are continuous and strictly increasing functions from $I$ to $\mathbb{R}_{+}$, with $f_{i}(0)=g(0)=0$ and $\delta \in(0,1]$ is a discount factor. The game has a finite horizon $T$, so the receiver must quit at period $T$ if the game reaches period $T$. We denote by $\mathcal{G}_{n}$ a sender-receiver stopping game with $n$ senders.

If $\delta=1$, we say that the game has undiscounted payoffs. If $\delta \in(0,1)$, we say that the game has discounted payoffs with discount factor $\delta$. For convenience, we denote by $f_{i}^{t}\left(\theta^{t}\right)$ and $g^{t}\left(\theta^{t}\right)$ the payoff for the sender $i$ and the receiver respectively if the game ends at period $t$. Hence, $f_{i}^{t}(\theta)=\delta^{t-1} \cdot f_{i}(\theta)$ and $g^{t}(\theta)=\delta^{t-1} \cdot g(\theta)$. 


\subsubsection{Strategies}

In this chapter, we only consider Markov strategies, i.e. strategies which at any period $t$, do not take into account the realizations of states and the messages at periods $1,2, \ldots, t-1$.

A strategy $\sigma_{i}=\left(\sigma_{i}^{t}\right)_{t=1}^{T-1}$ for the sender $i$ is a sequence of measurable functions $\sigma_{i}^{t}: I \rightarrow[0,1]$. The interpretation is that, at each period $t<$ $T$, given the state $\theta^{t}$, the strategy $\sigma_{i}$ places probability $\sigma_{i}^{t}\left(\theta^{t}\right)$ on the message $m_{c}$ and remaining probability $1-\sigma_{i}^{t}\left(\theta^{t}\right)$ on the message $m_{q}$. For simplicity we require that at period $T$, the senders' strategy must send the message $m_{q}$.

A strategy $\tau=\left(\tau^{t}\right)_{t=1}^{T-1}$ for the receiver is a sequence of functions $\tau^{t}:\left\{m_{c}, m_{q}\right\}^{N} \rightarrow[0,1]$. We do not need any measurability conditions for $\tau^{t}$ as the domain of $\tau^{t}$ is finite. The interpretation is that, at each period $t$, given the message profile $m^{t}$, the strategy $\tau$ places probability $\tau^{t}\left(m^{t}\right)$ on the action $a_{c}$ and remaining probability $1-\tau^{t}\left(m^{t}\right)$ on the action $a_{q}$. We require that at period $T$, the receiver's strategy must play the action $a_{q}$.

We denote by $(\sigma, \tau)$ the strategy profile, i.e. $(\sigma, \tau)=\left(\sigma_{1}, \sigma_{2}, \ldots, \sigma_{n}, \tau\right)$. Denote by $\left(\hat{\sigma}_{i}, \sigma_{-i}, \tau\right)$ the profile in which sender $i$ deviates from his strategy $\sigma_{i}$ to $\hat{\sigma}_{i}$, i.e. $\left(\hat{\sigma}_{i}, \sigma_{-i}, \tau\right)=\left(\sigma_{1}, \ldots, \sigma_{i-1}, \hat{\sigma}_{i}, \sigma_{i+1}, \ldots, \sigma_{n}, \tau\right)$. Similarly denote by $\left(\hat{\sigma_{S}}, \sigma_{N \backslash S}, \tau\right)$ the strategy profile in which each sender $i \in S$ deviates from $\sigma_{i}$ to $\hat{\sigma}_{i}$, where $S \subseteq N$.

\subsubsection{Perfect Bayesian equilibrium}

In this section we discuss the solution concept of perfect Bayesian equilibrium.

Consider a strategy profile $(\sigma, \tau)$. Let $S$ denote the random variable for the period when the receiver quits. The expected payoffs of the sender 
$i$ and the receiver are denoted by $U_{i}(\sigma, \tau)$ and $U_{r}(\sigma, \tau)$ respectively and defined as follows:

$$
U_{i}(\sigma, \tau)=\mathbb{E}_{(\sigma, \tau)}\left[f_{i}^{S}\left(\theta^{S}\right)\right], \quad U_{r}(\sigma, \tau)=\mathbb{E}_{(\sigma, \tau)}\left[g^{S}\left(\theta^{S}\right)\right],
$$

where $\mathbb{E}_{(\sigma, \tau)}$ is the expectation operator with respect to $(\sigma, \tau)$.

If the receiver has not quit until some period $t$, then the continuation expected payoffs from period $t$ onward are denoted by $U_{i}^{t}(\sigma, \tau)$ and $U_{r}^{t}(\sigma, \tau)$ respectively and defined as follows:

$$
\begin{aligned}
& U_{i}^{t}(\sigma, \tau)=\delta^{t-1} \cdot U_{i}\left(\sigma_{1}[t], \ldots, \sigma_{n}[t], \tau[t]\right), \\
& U_{r}^{t}(\sigma, \tau)=\delta^{t-1} \cdot U_{r}\left(\sigma_{1}[t], \ldots, \sigma_{n}[t], \tau[t]\right),
\end{aligned}
$$

where $\sigma_{i}[t]=\left(\sigma_{i}^{k}\right)_{k=t}^{T-1}$ and $\tau[t]=\left(\tau^{k}\right)_{k=t}^{T-1}$ are the continuation strategies of $\sigma_{i}$ and $\tau$ respectively.

Definition 3.1. A strategy profile $(\sigma, \tau)$ is called a Perfect Bayesian Equilibrium (PBE) if for every period $t$, we have $U_{i}^{t}\left(\sigma_{i}, \sigma_{-i}, \tau\right) \geq U_{i}^{t}\left(\sigma_{i}^{\prime}, \sigma_{-i}, \tau\right)$ for every $i$, for every strategy $\sigma_{i}^{\prime}$ of the sender $i$, and $U_{r}^{t}(\sigma, \tau) \geq U_{r}^{t}\left(\sigma, \tau^{\prime}\right)$ for every strategy $\tau^{\prime}$ of the receiver. ${ }^{3}$

We will regularly make use of the fact that the well-known one-shot deviation principle holds in our games (regardless whether the payoffs are undiscounted or discounted). More precisely, a strategy profile $(\sigma, \tau)$ is a PBE if and only if (1) for every $t$ and every $i$, we have $U_{i}^{t}(\sigma, \tau) \geq U_{i}^{t}\left(\hat{\sigma}_{i}, \sigma_{-i}, \tau\right)$ for every $\hat{\sigma}_{i}$ that is a one-shot deviation from $\sigma_{i}$ at period $t$, and (2) similarly for the receiver. Here, for two strategies $\sigma_{i}$ and $\hat{\sigma}_{i}$ and period $t, \hat{\sigma}_{i}$ is called a one-shot deviation from $\sigma_{i}$ at period $t$ if $\hat{\sigma}_{i}^{t} \neq \sigma_{i}^{t}$ and $\hat{\sigma}_{i}^{t^{\prime}}=\sigma_{i}^{t^{\prime}}$ for every period $t^{\prime} \neq t$. One-shot deviations are defined similarly for the receiver.

\footnotetext{
${ }^{3} \mathrm{PBE}$ is a refinement of Bayesian Nash Equilibrium (BNE). Intuitively, it requires that the strategy profile induces a BNE after any history.
} 


\subsubsection{Terminology for strategies}

In this section we define several classes of strategies for the senders and the receiver that play an important role in our analysis.

A strategy $\sigma_{i}$ for the sender $i$ is called pure if, for each period $t$ and state $\theta^{t}$, either $\sigma_{i}^{t}\left(\theta^{t}\right)=1$ or $\sigma_{i}^{t}\left(\theta^{t}\right)=0$. Pure strategies for the receiver are defined in a similar fashion.

A strategy $\sigma_{i}$ for the sender $i$ is called a threshold strategy, if for each $t<T$, there exists a threshold $\alpha_{i}^{t} \in[0,1]$ such that

$$
\sigma_{i}^{t}\left(\theta^{t}\right)= \begin{cases}1 & \text { if } \theta^{t} \in\left[0, \alpha_{i}^{t}\right) \\ 0 & \text { if } \theta^{t} \in\left(\alpha_{i}^{t}, 1\right] .\end{cases}
$$

We do not specify what a threshold strategy recommends when the state is exactly equal to the threshold, for the sake of flexible exposition of our results. In any case, this occurs with probability zero only. A strategy profile $(\sigma, \tau)$ is threshold strategy profile if $\sigma_{i}$ is a threshold strategy for each $i$.

A strategy $\tau$ of the receiver is anonymous if at each period, the receiver takes the action $a_{c}$ or the action $a_{q}$ depending only on the set of $n$ messages, and thus not on the identities of the senders who sent the messages. To make this definition more precise, define the count of a message profile $m^{t}$ by $C\left(m^{t}\right)=\left|\left\{i: m_{i}^{t}=m_{q}\right\}\right|$, which is the number of messages $m_{q}$ in the message profile. So, $\tau$ is anonymous if for each period $t$ and any two message profiles $m^{t}, \bar{m}^{t} \in\left\{m_{c}, m_{q}\right\}^{N}$, $C\left(m^{t}\right)=C\left(\bar{m}^{t}\right)$ implies $\tau^{t}\left(m^{t}\right)=\tau^{t}\left(\bar{m}^{t}\right)$.

A strategy $\tau$ of the receiver is monotonic if at each period $t$, we have $\tau^{t}\left(\bar{m}^{t}\right) \geq \tau^{t}\left(m^{t}\right)$ for any two message profiles $m^{t}$ and $\bar{m}^{t}$ which satisfy for each $i \in N, m_{i}^{t}=m_{c} \Rightarrow \bar{m}_{i}^{t}=m_{c}$.

A strategy $\tau$ of the receiver is monotonically anonymous if for each period $t$, there exists $n^{t} \in\{0,1, \ldots, n, n+1\}$ such that for each message profile 
$m^{t}$

$$
\tau^{t}\left(m^{t}\right)= \begin{cases}1 & \text { if } C\left(m^{t}\right)<n^{t} \\ 0 & \text { if } C\left(m^{t}\right) \geq n^{t}\end{cases}
$$

The number $n^{t}$ is the threshold of $\tau$ at period $t$. Note that if $\tau$ is monotonically anonymous, then it is both anonymous and monotonic, while the the converse is not true.

A strategy $\tau$ of the receiver is babbling if for some $t<T$, we have $\tau^{t}\left(m^{t}\right)=\tau^{t}\left(\bar{m}^{t}\right)$ for any two message profiles $m^{t}, \bar{m}^{t}$. Clearly, a monotonically anonymous strategy $\tau$ is babbling if and only if the threshold $n^{t}$ is equal to 0 or $n+1$.

\subsection{Regular strategy profile}

\subsubsection{Sincere strategy of the senders}

Let $(\sigma, \tau)$ be a fixed strategy profile. The strategy $\sigma_{i}$ of the sender $i$ is said to be sincere against the strategy profile $(\sigma, \tau)$ if at each period $t<T, \sigma_{i}$ has a threshold $\alpha_{i}^{t}$ which is given by

$$
\alpha_{i}^{t}=\left(f_{i}^{t}\right)^{-1}\left(U_{i}^{t+1}(\sigma, \tau)\right) .
$$

Intuitively, this has the following meaning: the strategy $\sigma_{i}$ chooses message $m_{c}$ if the payoff $f_{i}^{t}\left(\theta^{t}\right)$ that the sender $i$ receives when the receiver quits is lower than the expected continuation payoff $U_{i}^{t+1}(\sigma, \tau)$, and chooses the message $m_{q}$ if it is higher.

More precisely, first consider the case $\theta^{t}<\alpha_{i}^{t}$. Then, by monotonicity we have $f_{i}^{t}\left(\theta^{t}\right)<f_{i}^{t}\left(\alpha_{i}^{t}\right)=U_{i}^{t+1}(\sigma, \tau)$. And indeed, as $\theta^{t}<\alpha_{i}^{t}$, the sincere strategy $\sigma_{i}$ chooses the message $m_{c}$. Now consider the case $\theta^{t}>\alpha_{i}^{t}$. Then, by monotonicity we have $f_{i}^{t}\left(\theta^{t}\right)>f_{i}^{t}\left(\alpha_{i}^{t}\right)=U_{i}^{t+1}(\sigma, \tau)$. And indeed, as $\theta^{t}>\alpha_{i}^{t}$, the sincere strategy $\sigma_{i}$ chooses the message $m_{q}$. 
The definition of $\alpha_{i}^{t}$ makes sense only if $U_{i}^{t+1}(\sigma, \tau)$ is an element of range of $f_{i}^{t}$, equivalently if the following inequalities are true: $f_{i}^{t}(0) \leq$ $U_{i}^{t+1}(\sigma, \tau) \leq f_{i}^{t}(1)$. The left inequality is trivially true, since $f_{i}^{t}(0)=0$ and the sender never gets negative payoff. The right inequality is also true, since if the receiver quits at any period $t^{\prime} \geq t+1$, the payoff obtained is at most $f_{i}^{t^{\prime}}(1) \leq f_{i}^{t+1}(1) \leq f_{i}^{t}(1)$. Hence, the inequalities are true. This also implies $0 \leq \alpha_{i}^{t} \leq 1$ at each period $t<T$ and for each $i$.

\subsubsection{Reactive strategy of the receiver}

Consider a strategy profile $(\sigma, \tau)$ in which the strategy $\sigma_{i}$ for each sender $i$ is a threshold strategy. Assume that $\sigma_{i}$ has the threshold $\alpha_{i}^{t}$ at period $t$. Let $\beta_{k}^{t}$ denote the $k^{\text {th }}$ lowest number from the set $\left\{\alpha_{1}^{t}, \alpha_{2}^{t}, \ldots, \alpha_{n}^{t}\right\}$. For the sake of notation, let $\beta_{0}^{t}=0$ and $\beta_{n+1}^{t}=1$. Hence, $\beta_{0}^{t} \leq \beta_{1}^{t} \leq \ldots \leq \beta_{n}^{t} \leq \beta_{n+1}^{t}$. For $0 \leq k \leq n$, if $\beta_{k}^{t}<\beta_{k+1}^{t}$, define the quantity

$$
G_{k}^{t}=\frac{1}{\beta_{k+1}^{t}-\beta_{k}^{t}} \int_{\beta_{k}^{t}}^{\beta_{k+1}^{t}} g^{t}(\theta) d \theta
$$

and if $\beta_{k}^{t}=\beta_{k+1}^{t}$, then define $G_{k}^{t}=g^{t}\left(\beta_{k}^{t}\right)$.

The interpretation of the term $G_{k}^{t}$ is as follows. For a fixed $t$ and $k$, assume that the realized state $\theta^{t}$ at period $t$ satisfies $\beta_{k}^{t}<\theta^{t}<\beta_{k+1}^{t}$. Then at this period, exactly $k$ senders will send the message $m_{q}$ and the remaining $n-k$ senders will send the message $m_{c}$. In other words, $C\left(m^{t}\right)=k$. Hence, assuming the receiver knows the strategies of the senders, he can conclude that $\beta_{k}^{t} \leq \theta^{t} \leq \beta_{k+1}^{t}$ on seeing the message profile $m^{t}$. So in this case, the quantity $G_{k}^{t}$ represents the expected payoff of the receiver on quitting.

As we have $\beta_{k}^{t} \leq \beta_{k+1}^{t}$ for $k=0,1, \ldots, n$, by Lemma 3.17, it follows that $G_{k}^{t} \leq G_{k+1}^{t}$ for $k=0,1, \ldots, n-1$ and equality occurs if and only if $\beta_{k}^{t}=\beta_{k+1}^{t}=\beta_{k+2}^{t}$. 
A strategy $\tau$ for the receiver is reactive against a threshold strategy profile $(\sigma, \tau)$ if the following hold:

[1] The strategy $\tau$ is monotonically anonymous.

[2] At each period $t$, the threshold $n^{t}$ of $\tau$ is the smallest $k$ for which $U_{r}^{t+1}(\sigma, \tau) \leq G_{k}^{t}$. If there is no such $k$, then $n^{t}=n+1$.

We now discuss the interpretation of condition [2] of reactive strategies. If $k<n^{t}$, then $U_{r}^{t+1}(\sigma, \tau)>G_{k}^{t}$. So, in this case, the receiver prefers to continue. Indeed, as $C\left(m^{t}\right)=k<n^{t}$, the strategy $\tau$ suggests action $a_{c}$. And if $k \geq n^{t}$, then we have $U_{r}^{t+1}(\sigma, \tau) \leq G_{k}^{t}$, so the receiver prefers to quit. Now as $C\left(m^{t}\right)=k \geq n^{t}$, the strategy $\tau$ suggests action $a_{q}$.

Denote by $\gamma^{t}=\beta_{n^{t}}^{t}$. By definition, $n^{t} \in\{0,1, \ldots, n, n+1\}$. If $n^{t}=0$, then according to $\tau$ the receiver always quits irrespective of the messages of the senders. Similarly if $n^{t}=n+1$, then the receiver always continues irrespective of the messages of the senders. These 'babbling' strategies are fairly uninteresting from a strategical perspective.

\subsubsection{Regular strategy profile}

A strategy profile $(\sigma, \tau)$ is regular if the following hold:

[1] For each sender $i$, the strategy $\sigma_{i}$ is sincere against $(\sigma, \tau)$.

[2] The receiver's strategy $\tau$ is reactive against $(\sigma, \tau)$.

We start by a constructing a strategy profile $(\tilde{\sigma}, \tilde{\tau})$ which we will use to show the existence and uniqueness of the regular strategy profile. We construct the strategies backward, starting at period $T$. As the requirement of the game, at period $T$, each sender must send the message $m_{q}$ irrespective of the state and the receiver must take the action $a_{q}$ irrespective of the messages. Hence, $U_{i}^{T}(\tilde{\sigma}, \tilde{\tau})=\int_{0}^{1} f_{i}^{T}(\theta) d \theta$ and $U_{r}^{T}(\tilde{\sigma}, \tilde{\tau})=\int_{0}^{1} g^{T}(\theta) d \theta$. 
Now fix a period $t<T$. Assume that for $t^{\prime}>t, \tilde{\sigma}_{i}^{t^{\prime}}$ and $\tilde{\tau}^{t^{\prime}}$ are fixed, and hence the expected continuation payoffs $U_{i}^{t^{\prime}}(\tilde{\sigma}, \tilde{\tau})$ and $U_{r}^{t^{\prime}}(\tilde{\sigma}, \tilde{\tau})$ are fixed. Now we construct the $\tilde{\sigma}_{i}^{t}$ for each $i$ and $\tilde{\tau}^{t}$ at period $t$.

For each $i \in N$, define the $\tilde{\sigma}_{i}^{t}$ as follows:

$$
\tilde{\sigma}_{i}^{t}\left(\theta^{t}\right)= \begin{cases}1 & \text { if } \theta^{t}<\tilde{\alpha}_{i}^{t} \\ 0 & \text { if } \theta^{t}>\tilde{\alpha}_{i}^{t}\end{cases}
$$

where the threshold $\tilde{\alpha}_{i}^{t}=\left(f_{i}^{t}\right)^{-1}\left(U_{i}^{t+1}(\tilde{\sigma}, \tilde{\tau})\right)$. We do not specify $\tilde{\sigma}_{i}^{t}\left(\tilde{\alpha}^{t}\right)$ and it can take any value in $[0,1]$.

Now that the thresholds $\tilde{\alpha}_{1}^{t}, \ldots, \tilde{\alpha}_{n}^{t}$ are fixed, define the numbers $\tilde{\beta}_{k}^{t}$ for $k=0,1, \ldots, n+1$, and $\tilde{G}_{k}^{t}$ for $k=0,1, \ldots, n$ as we did in subsection 3.3.2. For $t<T$, define $\tilde{n}^{t}$ to be the smallest $k$ for which $U_{r}^{t+1}(\tilde{\sigma}, \tilde{\tau}) \leq$ $\tilde{G}_{k}^{t}$ and if there is no such $k$, then $\tilde{n}^{t}=n+1$. Fix $\tilde{\tau}^{t}$ such that $\tilde{\tau}^{t}\left(m^{t}\right)=1$ if $C\left(m^{t}\right)<\tilde{n}^{t}$ and $\tilde{\tau}^{t}\left(m^{t}\right)=0$ if $C\left(m^{t}\right) \geq \tilde{n}^{t}$. Hence, $\tilde{\tau}$ is monotonically anonymous and has the threshold $\tilde{n}^{t}$ at each period $t<T$.

Now we prove by using backward induction that the above defined strategy profile is (essentially) unique regular strategy profile.

Proposition 3.2. The strategy profile $(\tilde{\sigma}, \tilde{\tau})$ is the (essentially) unique regular strategy profile.

Proof. By definition, it can be easily seen that against the profile $(\tilde{\sigma}, \tilde{\tau}), \tilde{\sigma}_{i}$ is sincere for each $i$ and $\tilde{\tau}$ reactive. Hence $(\tilde{\sigma}, \tilde{\tau})$ is a regular strategy profile. To prove the (essential) uniqueness, consider any regular strategy profile $(\sigma, \tau)$, so $\sigma_{i}$ is sincere for each $i$ and $\tau$ reactive against $(\sigma, \tau)$. Let $\alpha_{i}^{t}$ be the threshold of $\sigma_{i}$ at period $t$. Define the numbers $\beta_{i}^{t}, G_{k}^{t}$ as we did in subsection 3.3.2. Let $n^{t}$ be the threshold of $\tau$ at period $t$ (note that as $(\sigma, \tau)$ is regular, $\tau$ is monotonically anonymous and has threshold at each period). We will show that for each $i, \alpha_{i}^{t}=\tilde{\alpha}_{i}^{t}$ for and $n^{t}=\tilde{n}^{t}$ for each $t<T$ by using backward induction on $t$. 
We have $U_{i}^{T}(\sigma, \tau)=\int_{0}^{1} f_{i}^{T}(\theta) d \theta$ and $U_{r}^{T}(\sigma, \tau)=\int_{0}^{1} g^{T}(\theta) d \theta$. As $\sigma_{i}$ is sincere against the profile $(\sigma, \tau)$, it has threshold $\alpha_{i}^{T-1}$ at period $T-1$ such that $\alpha_{i}^{T-1}=\left(f_{i}^{T-1}\right)^{-1}\left(U_{i}^{T}(\sigma, \tau)\right)$. Since, $U_{i}^{T}(\tilde{\sigma}, \tilde{\tau})=U_{i}^{T}(\sigma, \tau)$, $\tilde{\alpha}_{i}^{T-1}=\alpha_{i}^{T-1}$ for each $i$. This implies, $G_{k}^{T-1}=\tilde{G}_{k}^{T-1}$ for each $k$. And since $U_{r}^{T}(\tilde{\sigma}, \tilde{\tau})=U_{r}^{T}(\sigma, \tau)$, we have $n^{T-1}=\tilde{n}^{T-1}$. Hence, the induction hypothesis is true for $t=T-1$.

For a fixed $t$, assume that $\alpha_{i}^{t^{\prime}}=\tilde{\alpha}_{i}^{t^{\prime}}$ for and $n^{t^{\prime}}=\tilde{n}^{t^{\prime}}$ for each $t^{\prime} \geq t$. Hence we have $U_{i}^{t}(\tilde{\sigma}, \tilde{\tau})=U_{i}^{t}(\sigma, \tau)$ for each $i$ and $U_{r}^{t}(\tilde{\sigma}, \tilde{\tau})=U_{r}^{t}(\sigma, \tau)$. This fact and the definitions of the strategies $\tilde{\sigma}, \tilde{\tau}, \sigma, \tau$ imply $\tilde{\alpha}_{i}^{t-1}=$ $\alpha_{i}^{t-1}$ and $n^{t-1}=\tilde{n}^{t-1}$, which proves the induction hypothesis for $t-1$. Hence the proof is complete.

\subsection{Main results}

Our main results focus on the regular strategy profile. Our first result shows that the regular strategy profile is a PBE. In particular, this establishes the existence of PBE. Then we have two results about the properties about the regular strategy profile. First we show that the receiver's strategy is not babbling. And then we show that no coalition of senders can deviate to obtain strictly better payoffs.

\subsubsection{Preparations}

We fix a sender-receiver stopping game with $n \geq 1$ senders and with a finite horizon $T$. We start by deriving the expressions for the expected payoffs when the the players use the regular strategy profile $(\sigma, \tau)$.

For convention, define $\alpha_{i}^{T}=0, \gamma^{T}=0$, and $U_{i}^{T+1}(\sigma, \tau)=U_{r}^{T+1}(\sigma, \tau)=$ 0 for each $i$. Consider a period $t \leq T$. With probability $\gamma^{t}$, we have $\theta^{t}<\gamma^{t}=\beta_{n^{t}}^{t}$. Then strictly less than $n^{t}$ senders send the message $m_{q}$ and the receiver continues. In this case the sender $i$ gets the expected continuation payoff $U_{i}^{t+1}(\sigma, \tau)$. Similarly, with probability $1-\gamma^{t}$, we 
have $\theta^{t}>\gamma^{t}$. Then at least $n^{t}$ senders send the message $m_{q}$ and the receiver quits. In this case the sender $i$ gets the expected continuation payoff $\frac{1}{1-\gamma^{t}} \int_{\gamma^{t}}^{1} f_{i}^{t}(\theta) d \theta$. Then,

$$
U_{i}^{t}(\sigma, \tau)=\gamma^{t} \cdot U_{i}^{t+1}(\sigma, \tau)+\int_{\gamma^{t}}^{1} f_{i}^{t}(\theta) d \theta
$$

As $U_{i}^{t+1}(\sigma, \tau)=f_{i}^{t}\left(\alpha_{i}^{t}\right)$ for each $t \leq T$. Equation (3.1) can be rewritten as follows. For each $t \leq T$

$$
f_{i}^{t-1}\left(\alpha_{i}^{t-1}\right)=\gamma^{t} \cdot f_{i}^{t}\left(\alpha_{i}^{t}\right)+\int_{\gamma^{t}}^{1} f_{i}^{t}(\theta) d \theta
$$

The expression for the receiver's expected utility can be derived similarly. For each $t \leq T$

$$
U_{r}^{t}(\sigma, \tau)=\gamma^{t} \cdot U_{r}^{t+1}(\sigma, \tau)+\int_{\gamma^{t}}^{1} g^{t}(\theta) d \theta .
$$

Now we discuss some terminology that we use in our results. In this section we exclusively talk about the regular strategy profile $(\sigma, \tau)$, hence for brevity, we denote the expected continuation payoffs at period $t$ simply by $U_{i}^{t}$ and $U_{r}^{t}$ respectively.

By replacing $t$ by $t+1$, equation (3.2) can be rewritten as follows. For each $i$ and each $t<T$

$$
\alpha_{i}^{t}=f_{i}^{-1}\left(\delta \cdot\left[\gamma^{t+1} \cdot f_{i}\left(\alpha_{i}^{t+1}\right)+\int_{\gamma^{t+1}}^{1} f_{i}(\theta) d \theta\right]\right) .
$$


For each $i \in N$, define an auxiliary function $H_{i}: I \rightarrow I$ by

$$
H_{i}(x)=f_{i}^{-1}\left(\delta \cdot\left[x \cdot f_{i}(x)+\int_{x}^{1} f_{i}(\theta) d \theta\right]\right) .
$$

By part [2] of Lemma 3.15, the function $H_{i}$ has a unique fixed point $\zeta_{i} \in I$.

For each $i$, define the sequence of the numbers $\left(\zeta_{i}^{t}\right)_{t=1}^{T}$ as follows. First, $\zeta_{i}^{T}=0$. Then using a backwards iteration, $\zeta_{i}^{t}=H\left(\zeta_{i}^{t+1}\right)$ for all $t<T$. We have (by part [1] of Lemma 3.16), $0=\zeta_{i}^{T}<\zeta_{i}^{T-1}<\cdots<\zeta_{i}^{1}<1$.

Define the function $V:[0,1] \rightarrow \mathbb{R}$ by letting for every $x>0$

$$
V(x)=\frac{1}{x} \cdot \int_{0}^{x} g(\theta) d \theta
$$

and $V(0)=0$. The amount $V(x)$ is the expected payoff for the receiver if he quits, conditional on the state being in $[0, x]$. For $T \in \mathbb{N}$ and $i \in N$, let $D_{i}^{T}$ be the smallest number in $[0,1]$ such that $\delta \cdot V(1) \geq V\left(\zeta_{i}^{1}\right)$ for every $\delta \in\left(D_{i}^{T}, 1\right]$. The number $\zeta_{i}^{1}$ itself depends on the discount factor $\delta$. Hence, $D_{i}^{T}$ does not necessarily have a simple closed formula. As $\zeta_{i}^{1}<1$, the inequality $\delta \cdot V(1) \geq V\left(\zeta_{i}^{1}\right)$ is true for $\delta=1$. Hence $D_{i}^{T}$ exists and satisfies $D_{i}^{T} \leq 1$. Due to part [4] of Lemma 3.16, $D_{i}^{T}<1$. Define $D^{T}=\max \left\{D_{1}^{T}, \ldots, D_{n}^{T}\right\}$. So we have, $D^{T}<1$.

\subsubsection{Results}

Theorem 3.3. For $\delta \in(0,1]$, the regular strategy profile is a $P B E$.

Proof. Step 1. We show that for each sender $i$ at each period $t, \sigma_{i}$ is best response against $\left(\sigma_{-i}, \tau\right)$.

Proof of Step 1. Fix a sender $i$. Let $k$ be such that $\alpha_{i}^{t}=\beta_{k}^{t}$.

Case 1. $\alpha_{i}^{t}<\gamma^{t}$. 
Consider the subcase where $C\left(m^{t}\right)<n^{t}$. In this scenario, the receiver continues. As less than $n^{t}$ senders send the message $m_{q}$, at least $n-$ $n^{t}+1$ senders send the message $m_{c}$. So, there are at least $n-n^{t}+1$ senders with threshold greater than than or equal to $\theta^{t}$. This implies $\gamma^{t}=\beta_{n^{t}} \geq \theta^{t}$. As $\alpha_{i}^{t}<\gamma^{t}=\beta_{n^{t}}^{t}$, there there are at least $n-n^{t}+1$ senders with threshold higher than $i$. So, So, there are at least $n-n^{t}+1$ senders excluding $i$ with threshold greater than than or equal to $\theta^{t}$. Hence, the inequality will be true even if the sender $i$ change his message. So, the receiver continues irrespective of the message of sender $i$. Hence, $i$ is playing the best response.

As $\alpha_{i}^{t}<\gamma^{t}$, the sender $i$ is not one of these senders.

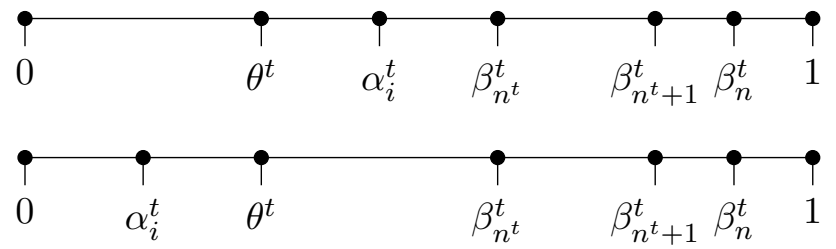

Now consider the subcase where $C\left(m^{t}\right) \geq n^{t}$. In this scenario, the receiver quits. As at least $n^{t}$ senders send the message $m_{q}$, there are at least $n^{t}$ senders with threshold less than or equal to $\theta^{t}$. This implies $\gamma^{t}=\beta_{n^{t}} \leq \theta^{t}$. As $\alpha_{i}^{t}<\gamma^{t} \leq \theta^{t}$, the sender $i$ also prefers the receiver to continue. As the sender $i$ obtains the desired action from the receiver, he is playing the best response.

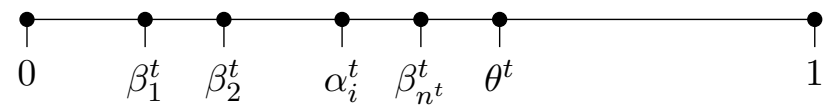

Case 2. $\alpha_{i}^{t}>\gamma^{t}$.

This case is completely analogous to case 1 .

Case 3. $\alpha_{i}^{t}=\gamma^{t}$.

If $\theta^{t}<\gamma^{t}$, then less than $n^{t}$ senders send the message $m_{q}$. So, $C\left(m^{t}\right)<$ $n^{t}$ and the receiver continues. As $\alpha_{i}^{t}=\gamma^{t}>\theta^{t}$, the sender $i$ also prefers 
that the receiver continues. If $\theta^{t}>\gamma^{t}$, then at least $n^{t}$ senders send the message $m_{q}$. So, $C\left(m^{t}\right) \geq n^{t}$ and the receiver quits. As $\alpha_{i}^{t}=\gamma^{t}<\theta^{t}$, the sender $i$ also prefers that the receiver quits. If $\theta^{t}=\gamma^{t}$, i is indifferent between any of the receiver's actions. In all these three cases, as the sender $i$ obtains the desired action from the receiver, he is playing the best response.

Now we will prove that $\tau$ is a best response against $\sigma=\left(\sigma_{1}, \ldots, \sigma_{n}\right)$. For that, we will show that at each period $t$, the receiver prefers to continue if $C\left(m^{t}\right)<n^{t}$ and to quit if $C\left(m^{t}\right) \geq n^{t}$.

Step 2. At each period $t$ for the receiver, continuing is a best response to $\sigma$ if $C\left(m^{t}\right)<n^{t}$ and quitting is the best response to $\sigma$ if $C\left(m^{t}\right) \geq n^{t}$.

Proof of Step 2. Fix a message profile $m^{t}$ and denote by $n^{\prime}=C\left(m^{t}\right)$. As $\beta_{1}^{t} \leq \ldots \leq \beta_{n}^{t}$, the senders corresponding to the threshold values $\beta_{1}^{t}, \ldots, \beta_{n^{\prime}}^{t}$, send the message $m_{q}$ and corresponding to the threshold values $\beta_{n^{\prime}+1}^{t^{\prime}}, \ldots, \beta_{n}^{t}$, send the message $m_{c}$. Hence, the realized state $\theta^{t}$ satisfies $\beta_{n^{\prime}}^{t} \leq \theta^{t} \leq \beta_{n^{\prime}+1}^{t}$. So, on seeing the message profile $m^{t}$, the expected utility for the receiver on quitting is $G_{n^{\prime}}^{t}$, while the expected utility for the receiver on continuing is $U_{r}^{t+1}(\sigma, \tau)$. So the receiver prefers to continue if $U_{r}^{t+1} \geq G_{n^{\prime}}^{t}$ and to quit if $U_{r}^{t+1} \leq G_{n^{\prime}}^{t}$. By definition of $n^{t}$, we have $U_{r}^{t+1} \leq G_{k}^{t}$ if $k \geq n^{t}$ and $U_{r}^{t+1}>G_{k}^{t}$ if $k<n^{t}$. So if $C\left(m^{t}\right)=n^{\prime}<n^{t}$, then $U_{r}^{t+1}>G_{n^{\prime}}^{t}$ so the receiver prefers to continue. On the other hand, if $C\left(m^{t}\right)=n^{\prime} \geq n^{t}$, then $U_{r}^{t+1} \leq G_{n^{\prime}}^{t}$, so the receiver prefers to quit.

Theorem 3.4. If $\delta \geq D^{T}$, then in the regular strategy profile, the receiver's strategy is not babbling.

Proof. Consider the regular strategy profile $(\sigma, \tau)$. As $\tau$ is reactive against $(\sigma, \tau)$, it is babbling if and only if its threshold $n^{t}$ is equal to 0 or $n+1$ at some period $t$. So it is sufficient to show that $1 \leq n^{t} \leq n$ at each period $t$.

Step 1. For each $i$ and $t<T$, we have $0<\alpha_{i}^{t}<1$. 
Proof of Step 1. The proof of the Lemma is by backward induction on $t$. We have $U_{i}^{T}(\sigma, \tau)=\int_{0}^{1} f_{i}^{T}(\theta) d \theta$. So, $f_{i}^{T}(0)<U_{i}^{T}(\sigma, \tau)<f_{i}^{T}(1)$ and hence, $0<\alpha_{i}^{T-1}<1$. This proves the statement for $t=T-1$. Assume that the statement is true for a fixed $t$, hence $0<\alpha_{i}^{t}<1$. We need to prove that $0<\alpha_{i}^{t-1}<1$, or equivalently $f_{i}^{t-1}(0)<f_{i}^{t-1}\left(\alpha_{i}^{t}\right)<f_{i}^{t-1}(1)$. We have from equation (3.2),

$$
f_{i}^{t-1}\left(\alpha_{i}^{t-1}\right)=\gamma^{t} \cdot f_{i}^{t}\left(\alpha_{i}^{t}\right)+\int_{\gamma^{t}}^{1} f_{i}^{t}(\theta) d \theta .
$$

Both terms in the RHS are non-negative. Moreover, If $\gamma^{t}>0$, then first term in the RHS is positive and if $\gamma^{t}=0$, the second term in the RHS is positive. So, $f_{i}^{t-1}\left(\alpha_{i}^{t}\right)>0=f_{i}^{t-1}(0)$. Furthermore if $\gamma^{t}>0$, we have RHS $<\gamma^{t} \cdot f_{i}^{t}(1)+\left(1-\gamma^{t}\right) \cdot f_{i}^{t}(1) d \theta=f_{i}^{t}(1) \leq f_{i}^{t-1}(1)$. And if $\gamma^{t}=0$, RHS $=\int_{0}^{1} f_{i}^{t}(\theta) d \theta<f_{i}^{t}(1) \leq f_{i}^{t-1}(1)$. This completes the proof of Step 1.

We have $0<\alpha_{i}^{t-1}<1$ for each $i$ and $t<T$. As a consequence, for each $t<T$ the following three statements are equivalent: (1) $n^{t} \leq n$, (2) $\gamma^{t}<1$, (3) $U_{r}^{t+1} \leq G_{n}^{t}$. Similarly the following three statements are also equivalent: (1) $n^{t} \geq 1$, (2) $\gamma^{t}>0$, (3) $U_{r}^{t+1}>G_{0}^{t}$.

Step 2. For each $i$ and each period $t \leq T$, we have $\alpha_{i}^{t} \leq \zeta_{i}^{t}$ and $\alpha_{i}^{t}<$ $H_{i}\left(\alpha_{i}^{t}\right)$.

Proof of Step 2. For any fixed $i$, we shall prove that for each $t \leq T$, $\alpha_{i}^{t} \leq \zeta_{i}^{t}$, by using backward induction on $t$. Firstly, $0=\alpha_{i}^{T} \leq \zeta_{i}^{T}=0$. Now assume that for some $t$, the following is true: $\alpha_{i}^{t^{\prime}} \leq \zeta_{i}^{t^{\prime}}$ for each $t^{\prime}>t$. We will show that $\alpha_{i}^{t} \leq \zeta_{i}^{t}$. We have from equation (3.4), 


$$
\begin{aligned}
\alpha_{i}^{t} & =f_{i}^{-1}\left(\delta \cdot\left[\gamma^{t+1} \cdot f_{i}\left(\alpha_{i}^{t+1}\right)+\int_{\gamma^{t+1}}^{1} f_{i}(\theta) d \theta\right]\right) \\
& \leq f_{i}^{-1}\left(\delta \cdot\left[\alpha_{i}^{t+1} \cdot f_{i}\left(\alpha_{i}^{t+1}\right)+\int_{\alpha_{i}^{t+1}}^{1} f_{i}(\theta) d \theta\right]\right) \\
& =H_{i}\left(\alpha_{i}^{t+1}\right) \leq H_{i}\left(\zeta_{i}^{t+1}\right)=\zeta_{i}^{t} .
\end{aligned}
$$

The first inequality follows from the fact that the auxiliary function defined below is maximized at $x=\alpha_{i}^{t+1}$.

$$
K_{i}(x)=f_{i}^{-1}\left(\delta\left[x \cdot f_{i}\left(\alpha_{i}^{t+1}\right)+\int_{x}^{1} f_{i}(\theta) d \theta\right]\right) .
$$

The second inequality follows due to the inductive hypothesis: $\alpha_{i}^{t+1} \leq$ $\zeta_{i}^{t+1}$. Hence, $\alpha_{i}^{t} \leq \zeta_{i}^{t}$. This completes the proof of first part of Step 2 . By part [1] of Lemma 3.16, we have $\zeta_{i}^{t}<\zeta_{i}$. This implies $\alpha_{i}^{t}<\zeta_{i}$ and equivalently, $\alpha_{i}^{t}<H_{i}\left(\alpha_{i}^{t}\right)$ (by part [3] of Lemma 3.15). This completes the proof of the Step 2.

Step 3. For each period $t<T$, if $\gamma^{t+1}<1$, then $\gamma^{t+1} \leq \beta_{n}^{t}$.

Proof of Step 3. Assume $t<T$ and $\gamma^{t+1}<1$. Then there is a sender $i$ such that $\gamma^{t+1}=\alpha_{i}^{t+1}$. We have from equation (3.4),

$$
\begin{aligned}
\alpha_{i}^{t} & =f_{i}^{-1}\left(\delta\left[\gamma^{t+1} \cdot f_{i}\left(\alpha_{i}^{t+1}\right)+\int_{\gamma^{t+1}}^{1} f_{i}(\theta) d \theta\right]\right) \\
& =f_{i}^{-1}\left(\delta\left[\alpha_{i}^{t+1} \cdot f_{i}\left(\alpha_{i}^{t+1}\right)+\int_{\alpha_{i}^{t+1}}^{1} f_{i}(\theta) d \theta\right]\right) \\
& =H_{i}\left(\alpha_{i}^{t+1}\right)>\alpha_{i}^{t+1} .
\end{aligned}
$$


The second equality follows from the fact that $\gamma^{t+1}=\alpha_{i}^{t+1}$ and the inequality follows from Step 2. So, we have $\gamma^{t+1}=\alpha_{i}^{t+1} \leq \alpha_{i}^{t} \leq \beta_{n}^{t}$. This completes the proof of Step 3.

Step 4. For each period $t<T$, if $\gamma^{t}<1$, then $U_{r}^{t+1} \leq \frac{1}{1-\gamma^{t}} \int_{\gamma^{t}}^{1} g^{t}(\theta) d \theta$.

Proof of Step 4. Assume $t<T$ and $\gamma^{t}<1$. Equivalently, $n^{t} \leq n$. So, $\beta_{n^{t}+1}^{t}$ is well defined and $\beta_{n^{t}+1}^{t} \leq 1$. By definition of $n^{t}$, we get $U_{r}^{t+1} \leq G_{n^{t}}^{t}$. We have

$$
\begin{aligned}
G_{n^{t}}^{t} & =\frac{1}{\beta_{n^{t}+1}^{t}-\beta_{n^{t}}^{t}} \int_{\beta_{n^{t}}^{t}}^{\beta_{n^{t}+1}^{t}} g^{t}(\theta) d \theta \\
& \leq \frac{1}{1-\beta_{n^{t}}^{t}} \int_{\beta_{n^{t}}^{t}}^{1} g^{t}(\theta) d \theta \\
& =\frac{1}{1-\gamma^{t}} \int_{\gamma^{t}}^{1} g^{t}(\theta) d \theta
\end{aligned}
$$

The inequality follows from the fact that $\beta_{n^{t}+1}^{t} \leq 1$. This completes the proof of Step 4.

Step 5. For each period $t<T$, we have $U_{r}^{t+1} \leq G_{n}^{t}$.

Proof of Step 5. The proof is using backward induction. First consider the base case $t=T-1$. We have $U_{r}^{T}=\int_{0}^{1} g^{T}(\theta) d \theta$ and $G_{n}^{T-1}=$ $\frac{1}{1-\beta_{n}^{T-1}} \int_{\beta_{n}^{T-1}}^{1} g^{T-1}(\theta) d \theta$. Hence $U_{r}^{T} \leq G_{n}^{T-1}$.

Now assume $U_{r}^{t+2} \leq G_{n}^{t+1}$ as induction hypothesis, then $n^{t+1} \leq n$ and $\gamma^{t+1}<1$. We will show that $U_{r}^{t+1} \leq G_{n}^{t}$. We have from equation (3.3), 


$$
\begin{aligned}
U_{r}^{t+1} & =\gamma^{t+1} \cdot U_{r}^{t+2}+\int_{\gamma^{t+1}}^{1} g^{t+1}(\theta) d \theta \\
& \leq \frac{\gamma^{t+1}}{1-\gamma^{t+1}} \int_{\gamma^{t+1}}^{1} g^{t+1}(\theta) d \theta+\int_{\gamma^{t+1}}^{1} g^{t+1}(\theta) d \theta \\
& =\frac{1}{1-\gamma^{t+1}} \int_{\gamma^{t+1}}^{1} g^{t+1}(\theta) d \theta \\
& \leq \frac{1}{1-\beta_{n}^{t}} \int_{\beta_{n}^{t}}^{1} g^{t}(\theta) d \theta \\
& =G_{n}^{t}
\end{aligned}
$$

The first inequality follows Step 4 and the fact that $\gamma^{t+1}<1$. The first inequality follows Step 3 and the fact that $\gamma^{t+1}<1$. This completes the proof of Step 5.

Step 6. For each period $t<T$, if $\gamma^{t}>0$, then $U_{r}^{t+1}>\frac{1}{\gamma^{t}} \int_{0}^{\gamma^{t}} g^{t}(\theta) d \theta$.

Proof of Step 6. Assume $t<T$ and $\gamma^{t}>1$. Equivalently, $n^{t} \geq 1$. So, $\beta_{n^{t}-1}^{t}$ is well defined and $\beta_{n^{t}-1}^{t} \geq 0$. By definition of $n^{t}$, we get $U_{r}^{t+1}>G_{n^{t}-1}^{t}$. We have

$$
\begin{aligned}
G_{n^{t}-1}^{t} & =\frac{1}{\beta_{n^{t}}^{t}-\beta_{n^{t}-1}^{t}} \int_{\beta_{n^{t}-1}^{t}}^{\beta_{n^{t}}^{t}} g^{t}(\theta) d \theta \\
& \geq \frac{1}{\beta_{n^{t}}^{t}-0} \int_{0}^{\beta_{n^{t}}^{t}} g^{t}(\theta) d \theta \\
& =\frac{1}{\gamma^{t}} \int_{0}^{\gamma^{t}} g^{t}(\theta) d \theta
\end{aligned}
$$

The inequality follows from the fact that $\beta_{n^{t}-1}^{t} \geq 0$. This completes the proof of Step 6.

Step 7. For each period $t<T$, we have $U_{r}^{t+1}>G_{0}^{t}$.

Proof of Step 7. The proof is by backward induction. Let $i$ be such that $\beta_{1}^{T-1}=\alpha_{i}^{T-1}$. By Step 2, we have $\alpha_{i}^{T-1} \leq \zeta_{i}^{T-1}$, and so $\beta_{1}^{T-1} \leq$ $\zeta_{i}^{T-1}$. We have 


$$
\begin{aligned}
U_{r}^{T} & =\int_{0}^{1} g^{T}(\theta) d \theta \\
& >\frac{1}{\zeta_{i}^{T-1}} \int_{0}^{\zeta_{i}^{T-1}} g^{T-1}(\theta) d \theta \\
& \geq \frac{1}{\beta_{1}^{T-1}} \int_{0}^{\beta_{1}^{T-1}} g^{T-1}(\theta) d \theta \\
& =G_{0}^{T-1} .
\end{aligned}
$$

First inequality follows from that fact $\beta_{1}^{T-1} \leq \zeta_{i}^{T-1}$. Second inequality follows from the assumption that $\delta \geq D^{T} \geq D_{i}^{T}$ and part [2] of Lemma 3.16. Hence $U_{r}^{t+1}>G_{0}^{t}$.

Now assume $U_{r}^{t+2}>G_{0}^{t+1}$ as induction hypothesis, then $n^{t+1} \geq 1$ and $\gamma^{t+1}>0$. We will show that $U_{r}^{t+1}>G_{0}^{t}$. Let $i$ be such that $\beta_{1}^{t}=\alpha_{i}^{t}$. By Step 2, we have $\alpha_{i}^{t} \leq \zeta_{i}^{t}$, and so $\beta_{1}^{t} \leq \zeta_{i}^{t}$. We have from equation (3.3),

$$
\begin{aligned}
U_{r}^{t+1} & =\gamma^{t+1} \cdot U_{r}^{t+2}+\int_{\gamma^{t+1}}^{1} g^{t+1}(\theta) d \theta \\
& >\gamma^{t+1} \cdot \frac{1}{\gamma^{t+1}} \int_{0}^{\gamma^{t+1}} g^{t+1}(\theta) d \theta+\int_{\gamma^{t+1}}^{1} g^{t+1}(\theta) d \theta \\
& =\int_{0}^{1} g^{t+1}(\theta) d \theta \\
& >\frac{1}{\zeta_{i}^{t}} \int_{0}^{\zeta_{i}^{t}} g^{t}(\theta) d \theta \\
& \geq \frac{1}{\beta_{1}^{t}} \int_{0}^{\beta_{1}^{t}} g^{t}(\theta) d \theta=G_{0}^{t} .
\end{aligned}
$$

First inequality follows from Step 6 and the fact that $\gamma^{t+1}>0$. Second inequality follows from the assumption that $\delta \geq D^{T} \geq D_{i}^{T}$ and part [2] of Lemma 3.16. Third inequality follows from the fact that $\beta_{1}^{t} \leq \zeta_{i}^{t}$.

Theorem 3.5. In the regular strategy profile, there does not exist a deviation by a coalition of senders which is strictly beneficial to each sender in the 
coalition.

Proof. Let $(\sigma, \tau)$ be the regular strategy profile. Assume by way of contradiction that there exists a coalition $S \subseteq N$ and strategies $\hat{\sigma}_{i}$ for each $i \in S$ such that $U_{i}^{t}\left(\hat{\sigma}_{S}, \sigma_{N \backslash S}, \tau\right)>U_{i}^{t}(\sigma, \tau)$ for each $i \in S$ and for some $t$. One-shot deviation principle applies here, so we can assume that for each $i \in S, \hat{\sigma}_{i}$ differs from $\sigma_{i}$ only at period $t$. Let $S^{\prime}$ be the set of senders not in $S$. Following the previous notation, let $n^{t}$ be the threshold of $\tau$ at period $t$ and $\alpha_{i}^{t}$ be the threshold of sender $\sigma_{i}$ at period $t$. Let $\beta_{k}^{t}$ be the $k^{\text {th }}$ smallest threshold. Without loss of generality, assume that $\alpha_{1}^{t} \leq \ldots \leq \alpha_{n}^{t}$. So, we have $\alpha_{i}^{t}=\beta_{i}^{t}$ for all $i$. Let $X_{S}=\left\{\alpha_{i}^{t}: i \in S\right\}$, and $X_{S^{\prime}}=\left\{\alpha_{i}^{t}: i \in S^{\prime}\right\}$.

Let $n_{2}$ be the $k^{\text {th }}$ smallest number from $X_{S^{\prime}}$ where $k=n^{t}$. If there is no such $k$, then let $n_{2}=1$. The interpretation of $n_{2}$ is as follows. There are at least $n^{t}$ senders in $S^{\prime}$ whose threshold is less than or equal to $\alpha_{n_{2}}^{t}$. So, if $\theta^{t}>\alpha_{n_{2}}^{t}$, there are at least $n^{t}$ senders in $S^{\prime}$ who send the message $m_{q}$. In this case the receiver quits even when the senders in $S$ deviate to any strategy $\hat{\sigma}_{S}$. However, if $\theta^{t}<\alpha_{n_{2}}^{t}$, the same cannot be said. For instance, if all the senders in $S$ deviate and send the message $m_{c}$, the total number of senders who send the message $m_{q}$ is strictly less than $n^{t}$, and so, the receiver continues.

Similarly, let $n_{1}$ be the $k^{\text {th }}$ largest number from $X_{S^{\prime}}$ where $k=n-$ $n^{t}+1$. If there is no such $k$, then let $n_{1}=0$. The interpretation of $n_{1}$ is as follows. There are at least $n-n^{t}+1$ senders in $S^{\prime}$ whose threshold is greater than or equal to $\alpha_{n_{1}}^{t}$. So, if $\theta^{t}<\alpha_{n_{2}}^{t}$, there are at least $n-n^{t}+1$ senders in $S^{\prime}$ who send the message $m_{c}$. In this case the receiver continues even when the senders in $S$ deviate to any strategy $\hat{\sigma}_{S}$. However, if $\theta^{t}>\alpha_{n_{2}}^{t}$, the same cannot be said. For instance, if all the senders in $S$ deviate and send the message $m_{q}$, the total number of senders who send the message $m_{q}$ is strictly greater than or equal to $n^{t}$, and so, the receiver quits.

Clearly, we have $n_{1} \leq n_{2}$. Moreover as $S$ is non-empty, we have $n_{1}<$ $n_{2}$. We are only interested when $S$ is nonempty, so we assume that 
$n_{1}<n_{2}$. Moreover, as $n_{1} \leq n^{t}$ and $n^{t} \leq n_{2}$, we have $\alpha_{n_{1}}^{t} \leq \gamma^{t} \leq \alpha_{n_{2}}^{t}$. Consider the strategy profile $\left(\hat{\sigma}_{S}, \sigma_{N \backslash S}, \tau\right)$. If $\theta^{t}<\alpha_{n_{1}}^{t}$ then the receiver continues irrespective of the messages by the senders in $S$ and if $\theta^{t}>$ $\alpha_{n_{2}}^{t}$ then the receiver quits irrespective of the messages by the senders in $S$. So, we can assume without loss of generality that $\hat{\sigma}_{i}^{t}\left(\theta^{t}\right)=\sigma_{i}^{t}\left(\theta^{t}\right)$ for each $i \in S$ and for $\theta^{t}<\alpha_{n_{1}}^{t}$ or $\theta^{t}>\alpha_{n_{2}}^{t}$.

Now assume that $\alpha_{n_{1}}^{t}<\theta^{t}<\alpha_{n_{2}}^{t}$. If all the senders in $S$ send the message $m_{c}$, then as $\theta^{t}<n_{2}$, overall strictly less than $n^{t}$ senders send the message $m_{q}$ and so, the receiver continues. However, if all the senders in $S$ send the message $m_{q}$, then as $\theta^{t}>\alpha_{n_{1}}^{t}$, overall at least $n^{t}$ senders send the message $m_{q}$ and so, the receiver quits. So, in the case where $\alpha_{n_{1}}^{t}<\theta^{t}<\alpha_{n_{2}}^{t}$, the coalition $S$ can determine the outcome of the receiver at period $t$ by choosing appropriate messages.

More specifically, consider the partition of the interval $I=\left(\alpha_{n_{1}}^{t}, \alpha_{n_{2}}^{t}\right)$ into sets $A$ and $B$ such that the messages of the senders induce the outcome where the receiver continues if $\theta^{t} \in A$ and quits if $\theta^{t} \in B$. Let $A_{1}=A \cap\left(\alpha_{n_{1}}^{t}, \gamma^{t}\right], A_{2}=A \cap\left(\gamma^{t}, \alpha_{n_{2}}^{t}\right), B_{1}=B \cap\left(\alpha_{n_{1}}^{t}, \gamma^{t}\right]$ and $B_{2}=B \cap\left(\gamma^{t}, \alpha_{n_{2}}^{t}\right)$. So we have $A_{1} \cup B_{1}=\left(\alpha_{n_{1}}^{t}, \gamma^{t}\right]$ and $A_{2} \cup B_{2}=$ $\left(\gamma^{t}, \alpha_{n_{2}}^{t}\right)$. This implies, $\left|A_{1}\right|+\left|B_{1}\right|=\gamma^{t}-\alpha_{n_{1}}^{t}$ and $\left|A_{2}\right|+\left|B_{2}\right|=\alpha_{n_{2}}^{t}-\gamma^{t}$. Also, $A_{1} \cup A_{2}=A$ and $B_{1} \cup B_{2}=B$, so $A_{1} \cup B_{1} \cup A_{2} \cup B_{2}=\left(\alpha_{n_{1}}^{t}, \alpha_{n_{2}}^{t}\right)$. This implies either (1) $\left|A_{1}\right|+\left|A_{2}\right| \geq \gamma^{t}-\alpha_{n_{1}}^{t}$ or (2) $\left|B_{1}\right|+\left|B_{2}\right| \geq \alpha_{n_{2}}^{t}-\gamma^{t}$.

Assume the case where (1) $\left|A_{1}\right|+\left|A_{2}\right| \geq \gamma^{t}-\alpha_{n_{1}}^{t}$. We will show a contradiction. The identical also for the case (2). As $\left|A_{1}\right|+\left|B_{1}\right|=\gamma^{t}-\alpha_{n_{1}}^{t}$, we have $\left|A_{2}\right| \geq\left|B_{1}\right|$.

$\underline{\text { Subcase } 1}$. There exists a sender $i \in S$ such that $\alpha_{i}^{t} \leq \gamma^{t}$.

Fix such a sender $i$. Consider a sender $i \in S$. Expected payoff for the sender $i$ at period $t$ in the regular strategy profile $(\sigma, \tau)$ is given by

$U_{i}^{t}(\sigma, \tau)=n_{1}^{t} \cdot U_{i}^{t+1}(\sigma, \tau)+\left(\gamma^{t}-n_{1}^{t}\right) \cdot U_{i}^{t+1}(\sigma, \tau)+\int_{\gamma^{t}}^{n_{2}^{t}} f_{i}^{t}(\theta) d \theta+\int_{n_{2}^{t}}^{1} f_{i}^{t}(\theta) d \theta$. 
Expected payoff for the sender $i$ at period $t$ in the strategy profile $\left(\sigma_{N \backslash S}, \hat{\sigma}_{S}, \tau\right)$ is given by

$$
\begin{aligned}
U_{i}^{t}\left(\sigma_{N \backslash S}, \hat{\sigma}_{S}, \tau\right)= & n_{1}^{t} \cdot U_{i}^{t+1}\left(\sigma_{N \backslash S}, \hat{\sigma}_{S}, \tau\right)+\left(\left|A_{1}\right|+\left|A_{2}\right|\right) \cdot U_{i}^{t+1}\left(\sigma_{N \backslash S}, \hat{\sigma}_{S}, \tau\right) \\
& +\int_{B_{1} \cup B_{2}} f_{i}^{t}(\theta) d \theta+\int_{n_{2}^{t}}^{1} f_{i}^{t}(\theta) d \theta .
\end{aligned}
$$

The profiles $(\sigma, \tau)$ and $\left(\sigma_{N \backslash S}, \hat{\sigma}_{S}, \tau\right)$ differ only at period $t$ and the expected payoffs at period $t+1$ are independent from the past strategies. Hence, $U_{i}^{t+1}\left(\sigma_{N \backslash S}, \hat{\sigma}_{S}, \tau\right)=U_{i}^{t+1}(\sigma, \tau)=f_{i}^{t}\left(\alpha_{n_{1}}^{t}\right)$.

We have $U_{i}^{t}\left(\sigma_{N \backslash S}, \hat{\sigma}_{S}, \tau\right)>U_{i}^{t}(\sigma, \tau)$ for all $i \in S$. Hence, we have

$$
\begin{aligned}
& \left(\left|A_{1}\right|+\left|A_{2}\right|\right) \cdot f_{i}^{t}\left(\alpha_{i}^{t}\right)+\int_{B_{1} \cup B_{2}} f_{i}^{t}(\theta) d \theta> \\
& \left(\gamma^{t}-n_{1}^{t}\right) \cdot f_{i}^{t}\left(\alpha_{i}^{t}\right)+\int_{\gamma^{t}}^{n_{2}^{t}} f_{i}^{t}(\theta) d \theta . \\
& \left(\left|A_{1}\right|+\left|A_{2}\right|\right) \cdot f_{i}^{t}\left(\alpha_{i}^{t}\right)+\int_{B_{1} \cup B_{2}} f_{i}^{t}(\theta) d \theta> \\
& \left(\left|A_{1}\right|+\left|B_{1}\right|\right) \cdot f_{i}^{t}\left(\alpha_{i}^{t}\right)+\int_{A_{2} \cup B_{2}} f_{i}^{t}(\theta) d \theta . \\
& \left(\left|A_{2}\right|\right) \cdot f_{i}^{t}\left(\alpha_{i}^{t}\right)+\int_{B_{1}} f_{i}^{t}(\theta) d \theta>\left(\left|B_{1}\right|\right) \cdot f_{i}^{t}\left(\alpha_{i}^{t}\right)+\int_{A_{2}} f_{i}^{t}(\theta) d \theta . \quad(*)
\end{aligned}
$$

As $B_{1} \subseteq\left(\alpha_{n_{1}}^{t}, \gamma^{t}\right]$, we have $\int_{B_{1}} f_{i}^{t}(\theta) d \theta \leq\left(\left|B_{1}\right|\right) \cdot f_{i}^{t}\left(\gamma^{t}\right)$. Similarly as $A_{2} \subseteq\left(\gamma^{t}, \alpha_{n_{2}}^{t}\right]$, we have $\int_{A_{2}} f_{i}^{t}(\theta) d \theta>\left(\left|A_{2}\right|\right) \cdot f_{i}^{t}\left(\gamma^{t}\right)$. So the previous equation implies 


$$
\begin{aligned}
& \left(\left|A_{2}\right|\right) \cdot f_{i}^{t}\left(\alpha_{i}^{t}\right)+\left(\left|B_{1}\right|\right) \cdot f_{i}^{t}\left(\gamma^{t}\right)>\left(\left|B_{1}\right|\right) \cdot f_{i}^{t}\left(\alpha_{i}^{t}\right)+\left(\left|A_{2}\right|\right) \cdot f_{i}^{t}\left(\gamma^{t}\right) . \\
& \left(\left|A_{2}\right|-\left|B_{1}\right|\right) \cdot\left(f_{i}^{t}\left(\alpha_{i}^{t}\right)-f_{i}^{t}\left(\gamma^{t}\right)\right)>0 .
\end{aligned}
$$

This is a contradiction, since we have $\left|A_{2}\right| \geq\left|B_{1}\right|$ and $\alpha_{i}^{t} \leq \gamma^{t}$.

Subcase 2. For all $i \in S$, we have $\gamma^{t}<\alpha_{i}^{t}$. Then we have, $n_{2}=n^{t}$. This implies, $\alpha_{n_{2}}^{t}=\gamma^{t}$. Then by definition, $A_{2}=B_{2}=\phi$ and $A_{1}=A$, $B_{1}=B$. Then the equation $\left.{ }^{*}\right)$ can be rewritten as $\int_{B_{1}} f_{i}^{t}(\theta) d \theta>\left(\left|B_{1}\right|\right)$. $f_{i}^{t}\left(\alpha_{i}^{t}\right)$. But as $B_{1} \subseteq\left(\alpha_{n_{1}}^{t}, \alpha_{n_{1}}^{t}\right)$, we have $\int_{B_{1}} f_{i}^{t}(\theta) d \theta \leq\left(\left|B_{1}\right|\right) \cdot f_{i}^{t}\left(\alpha_{n_{2}}^{t}\right)$. This is a contradiction, since $\alpha_{n_{2}}^{t}=\gamma^{t}<\alpha_{i}^{t}$.

\subsection{Illustrations and examples}

\subsubsection{An example: 2 senders}

In this section, we illustrate our concepts and results in detail. For ease of explanation, we consider the setting of only 2 senders and 1 receiver. Fix a game where the $f_{1}$ and $f_{2}$ and $g$ are the payoff functions of the two senders and the receiver respectively.

Let $(\sigma, \tau)$ be the regular strategy profile. Due to Theorem 3.3, $(\sigma, \tau)$ is a PBE. Following the notation from Section 3.3.2, let $\alpha_{1}^{t}$ and $\alpha_{2}^{t}$ denote the thresholds of the senders at a period $t$ and $U_{r}^{t+1}$ denotes the expected utility of the receiver at period $t+1$. As before, let $\beta_{1}^{t}=\min \left\{\alpha_{1}^{t}, \alpha_{2}^{t}\right\}$ and $\beta_{2}^{t}=\max \left\{\alpha_{1}^{t}, \alpha_{2}^{t}\right\}$. By definition, $G_{0}^{t}=\frac{1}{\beta_{1}^{t}} \int_{0}^{\beta_{1}^{t}} g^{t}(\theta) d \theta, G_{1}^{t}=$ $\frac{1}{\beta_{2}^{t}-\beta_{1}^{t}} \int_{\beta_{1}^{t}}^{\beta_{2}^{t}} g^{t}(\theta) d \theta$ and $G_{2}^{t}=\frac{1}{1-\beta_{2}^{t}} \int_{\beta_{2}^{t}}^{1} g^{t}(\theta) d \theta$. We have $G_{0}^{t} \leq G_{1}^{t} \leq$ $G_{2}^{t}$. Without loss of generality, assume $\alpha_{1}^{t} \leq \alpha_{2}^{t}$, hence $\alpha_{1}^{t}=\beta_{1}^{t}$ and $\alpha_{2}^{t}=\beta_{2}^{t}$. 
If we have $U_{r}^{t+1} \leq G_{0}^{t}$ then by definition of reactive strategy, the threshold for the receiver is $n^{t}=0$. Hence, the receiver quits whenever there are at least zero $m_{q}$ messages in the message profile. In other words, the receiver quits irrespective of the message profile. Hence in this case, $\tau$ is a babbling strategy. Due to Theorem 3.4, if $\delta \geq D^{T}$, this case cannot occur.

Now if $G_{0}^{t}<U_{r}^{t+1} \leq G_{1}^{t}$ then we have $n^{t}=1$. Hence, the receiver quits whenever there is at least one $m_{q}$ message. As $\alpha_{t}^{1} \leq \alpha_{2}^{t}$, the sender 1 always ${ }^{4}$ sends the message $m_{q}$ whenever sender 2 sends $m_{q}$. In other words, in this equilibrium, the receiver quits whenever the sender 1 quits. In this case we say that the receiver 'obeys' the sender 1 at period $t$.

Similarly in the scenario where $G_{1}^{t}<U_{r}^{t+1} \leq G_{2}^{t}$, we have $n^{t}=2$ and the receiver obeys the sender 2 at period $t$. Lastly when $G_{2}^{t}<U_{r}^{t+1}$, we have $n^{t}=3$, the receiver continues irrespective of the message profile, and hence plays a babbling strategy. So if $\delta \geq D^{T}$, this case cannot occur due to Theorem 3.4. Then, we have $1 \leq n^{t} \leq 2$. In the following example, we illustrate how we can compute the thresholds in the regular strategy profile.

Example 3.6. Let $f_{1}(\theta)=\frac{1}{2}\left(1+(2 \theta-1)^{3}\right), f_{2}(\theta)=\theta^{2}$ and $g(\theta)=\theta$ and $\delta=1$. As the receiver must quit at period $T$, the expected payoffs at period $T$ are $U_{1}^{T}=\int_{0}^{1} f_{1}^{T}(\theta) d \theta=\frac{1}{2}, U_{2}^{T}=\int_{0}^{1} f_{2}^{T}(\theta) d \theta=\frac{1}{3}$ and $U_{r}^{T}=\int_{0}^{1} g^{T}(\theta) d \theta=\frac{1}{2}$.

\footnotetext{
${ }^{4}$ Possibly except in the case where $\theta^{t}=\alpha_{1}^{t}=\alpha_{2}^{t}$, as the threshold strategy may send arbitrary message when the realized state equals the threshold. However, this case happens with probability zero only.
} 


\begin{tabular}{|c|c|c|c|c|c|}
\hline Period $(t)$ & $\alpha_{1}^{t}$ & $\alpha_{2}^{t}$ & $\beta_{1}^{t}$ & $\beta_{2}^{t}$ & $\gamma^{t}$ \\
\hline \hline$T$ & 0 & 0 & 0 & 0 & 0 \\
\hline$T-1$ & 0.5 & 0.58 & 0.5 & 0.58 & 0.5 \\
\hline$T-2$ & 0.75 & 0.68 & 0.68 & 0.75 & 0.68 \\
\hline
\end{tabular}

As the strategy $\sigma_{1}$ is sincere against $(\sigma, \tau)$, it has a threshold $\alpha_{1}^{T-1}=\left(f_{1}^{T-1}\right)^{-1}\left(U_{1}^{T}\right)=\frac{1}{2}$ at period $T-1$. Similarly $\sigma_{2}$ has the threshold $\alpha_{2}^{T-1}=\left(f_{2}^{T-1}\right)^{-1}\left(U_{2}^{T}\right)=\frac{1}{\sqrt{3}}$ at period $T-1$. As $\alpha_{1}^{T-1}<\alpha_{2}^{T-1}$, we have $\beta_{1}^{T-1}=\alpha_{1}^{T-1}=\frac{1}{2}$ and $\beta_{2}^{T-1}=\alpha_{2}^{T-1}=\frac{1}{\sqrt{3}}$. By definition, $\beta_{0}^{T-1}=0$ and $\beta_{3}^{T-1}=1$. We also have $G_{k}^{T-1}=\frac{1}{\beta_{k+1}^{T-1}-\beta_{k}^{T-1}} \int_{\beta_{k}^{T-1}}^{\beta_{k+1}^{T-1}} g^{T-1}(\theta) d \theta=\frac{\beta_{k}^{T-1}+\beta_{k+1}^{T-1}}{2}$. Substituting the values, we have $G_{0}^{T-1}=\frac{1}{4}=0.25$ and $G_{1}^{T-1}=\frac{\frac{1}{2}+\frac{1}{\sqrt{3}}}{2} \approx 0.54$.

As $G_{0}^{T-1}<U_{r}^{T} \leq G_{1}^{T-1}$, we have $n^{T-1}=1$. So, as $\beta_{1}^{T-1}$ corresponds to the threshold of sender 1 , the receiver obeys the sender 1 at period $T-1$. So, $\gamma^{T-1}=\alpha_{1}^{T-1}=\frac{1}{2}$.

The expected payoff at period $T-1$ for the receiver can be calculated using equation (3.3): $U_{r}^{T-1}=\gamma^{T-1} \cdot U_{r}^{T}+\int_{\gamma^{T-1}}^{1} g^{T-1}(\theta) d \theta=\frac{5}{8}$. Similarly, the expected payoff at period $T-1$ for the senders can be calculated using equation (3.1): $U_{1}^{T-1}=\frac{9}{16}$ and $U_{2}^{T-1}=\frac{11}{24}$. Hence, $\alpha_{1}^{T-2}=$ $\left(f_{1}^{T-2}\right)^{-1}\left(U_{1}^{T-1}\right)=0.75$ and $\alpha_{2}^{T-2}=\left(f_{2}^{T-2}\right)^{-1}\left(U_{2}^{T-1}\right)=\sqrt{\frac{11}{24}} \approx 0.68$. As $\alpha_{1}^{T-2}>\alpha_{2}^{T-2}, \beta_{1}^{T-2}=\alpha_{2}^{T-2}=0.68$ and $\beta_{2}^{T-2}=\alpha_{1}^{T-2}=0.75$. Again, $G_{k}^{T-2}=\frac{\beta_{k}^{T-2}+\beta_{k+1}^{T-2}}{2}$. Substituting the values, we get $G_{0}^{T-2}=$ 
$\frac{\sqrt{\frac{11}{24}}}{2} \approx 0.34$ and $G_{1}^{T-2}=\frac{\sqrt{\frac{11}{24}}+\frac{3}{4}}{2} \approx 0.71$.

As $G_{0}^{T-2}<U_{r}^{T-2} \leq G_{1}^{T-2}$, we have $n^{T-2}=1$. So, as $\beta_{1}^{T-2}$ corresponds to the threshold of sender 2 , the receiver obeys the sender 2 . So, $\gamma^{T-2}=$ $\alpha_{2}^{T-2}=\sqrt{\frac{11}{24}} \approx 0.68$.

\subsubsection{Other PBEs}

As shown by [1] in the setting of 1 sender and 1 receiver, the regular strategy profile is the unique PBE among the strategy profiles in which the receiver's strategy is not babbling. However, this is not true when we have more than 1 senders. In this subsection, we list some of the interesting non-babbling PBEs which are not regular strategy profiles.

In the following PBE, the receiver only considers the messages of sender 1 and ignores the other senders. In response, the other senders send completely uninformative messages.

Example 3.7. Take a game with at least 2 senders. Consider the following strategy profile. At each period $t$, the receiver continues if $m_{1}^{t}=m_{c}$ and quits if $m_{1}^{t}=m_{q}$. Sender 1 plays threshold strategy with thresholds $\alpha_{1}^{t}=\left(f_{1}^{t}\right)^{-1}\left(U_{1}^{t+1}(\sigma, \tau)\right)$ at each period $t<T$. Every other sender sends a constant message $m_{c}$ : for all $i \neq 1$ and for each $\theta^{t} \in I, \sigma_{i}^{t}\left(\theta^{t}\right)=1$.

Remark: The above example can be easily generalized. For $n^{\prime}<n$, we say that a game $\mathcal{G}_{n}$ is a extension of a game $\mathcal{G}_{n^{\prime}}$ if the receiver and the senders $i=1,2, \ldots, n^{\prime}$ have same payoff functions in $\mathcal{G}_{n}$ as in $\mathcal{G}_{n^{\prime}}$. Let $\left(\hat{\sigma_{1}}, \ldots \hat{\sigma_{n^{\prime}}}, \hat{\tau}\right)$ be any PBE in $\mathcal{G}_{n^{\prime}}$. Then $\left(\sigma_{1}, \ldots \sigma_{n}, \tau\right)$ is a PBE in $\mathcal{G}_{n}$ where the strategy profile $\left(\sigma_{1}, \ldots \sigma_{n}, \tau\right)$ is defined as follows. For $i=1, \ldots, n^{\prime}$, we have $\hat{\sigma}_{i}=\sigma_{i}$. For $i=n^{\prime}+1, \ldots, n$, we have $\sigma_{i}\left(\theta^{t}\right)=1$ 
for each $\theta^{t} \in I$ and each $t<T$. The strategy $\tau$ imitates the strategy $\hat{\tau}$ ignoring the messages of the senders $i=n^{\prime}+1, \ldots, n$.

In the following example, at each period, all the senders share the same thresholds. The receiver's strategy is stationary, since its threshold is the same for all the periods.

Example 3.8. Take a game with at least 2 senders. Consider the following strategy profile. Every senders plays a threshold strategy. The thresholds $\alpha_{i}^{t}$ are defined recursively as follows. At each period $t<T$, let $a_{i}^{t}=\left(f_{i}^{t}\right)^{-1}\left(U_{i}^{t+1}(\sigma, \tau)\right)$. Then for each $i, \alpha_{i}^{t}=\max _{j}\left\{a_{j}^{t}\right\}$. Moreover, if $\theta^{t}=\max _{j}\left\{a_{j}^{t}\right\}$, then at least one sender sends the message $m_{q}$. The receiver plays a reactive strategy with threshold $n^{t}=1$ for every period $t$.

In the following PBE, the senders 1, 2 and 3 play the threshold which is optimal for the receiver. While this may not be optimal outcome for any of these senders, none of them can unilaterally deviate to change the outcome, since the receiver obeys the majority of the three. In this $\mathrm{PBE}$, the receiver obtains maximum possible expected payoff.

Example 3.9. Take a game with at least 3 senders. Consider the following strategy profile. Senders 1, 2 and 3 play the threshold strategy with threshold $\alpha_{i}^{t}=\left(g^{t}\right)^{-1}\left(U_{r}^{t+1}(\sigma, \tau)\right)$ at each period $t$ and $i=1,2,3$. The receiver continues, if at least 2 of the 3 senders $i=1,2,3$ send the message $m_{c}$ and quits, if at least 2 of the 3 senders $i=1,2,3$ send the message $m_{q}$. All the remaining senders play arbitrary strategies.

\subsection{Externality of additional senders}

In this section, we discuss how the expected payoffs of the senders and the receiver are affected by having additional senders. Recall that 
a game $\mathcal{G}_{n+1}$ is a extension of a game $\mathcal{G}_{n}$ if the receiver and the senders $i=1,2, \ldots, n$ have same payoff functions in $\mathcal{G}_{n+1}$ as in $\mathcal{G}_{n}$.

Our goal is to study the effect of the sender $n+1$ on expected payoffs of the remaining players (senders $i=1,2, \ldots, n$ and the receiver) in game $\mathcal{G}_{n+1}$, compared to their expected payoffs in game $\mathcal{G}_{n}$. We consider the regular strategy profile in the respective games to be our focal equilibrium. We say that the sender $n+1$ imposes positive externality on a player (senders $i=1,2, \ldots, n$ and the receiver) if the expected payoff of that player in $\mathcal{G}_{n+1}$ is strictly higher than in $\mathcal{G}_{n}$ and imposes negative externality if the expected payoff of that player in $\mathcal{G}_{n+1}$ is strictly less than in $\mathcal{G}_{n}$.

\subsubsection{Externality of additional sender for the senders}

In the sender-receiver stopping game with only 1 sender as shown by [1], in the regular strategy profile, the receiver completely obeys the sender. Hence, the sender receives the best possible expected payoff among all the strategy profiles, not necessarily PBEs. Hence, the following proposition follows.

Proposition 3.10. Consider games $\mathcal{G}_{1}$ and $\mathcal{G}_{2}$, where $\mathcal{G}_{2}$ is a extension of $\mathcal{G}_{1}$. Then the sender 2 does not impose positive externality on sender 1 .

However a similar statement is not true in general if we consider games with higher number of senders. In general, an additional sender may have positive or negative externality on the current senders.

Proposition 3.11. For every $n \geq 2$, there exist games $\mathcal{G}_{n}$ and $\mathcal{G}_{n+1}$, where $\mathcal{G}_{n+1}$ is a extension of $\mathcal{G}_{n}$ such that sender $n+1$ imposes positive externality on some senders, while negative externality on others. 
Intuitively it is easy see that an additional sender can impose negative externality on existing senders. For the positive externality, one can think as follows. Consider a scenario where sender 2 is getting less expected payoffs due to the fact that receiver is obeying sender 1 and if the new sender 3 is introduced, the receiver obeys the sender 3 instead. If sender 2 prefers receiver obeying sender 3 than sender 1 , then sender 3 has positive externality on sender 2 . The example below illustrates this intuition and also serves as the proof of the Proposition 3.11 for $n=2$. Similar example can be constructed for any general $n$ by adding senders with same payoff functions.

Example 3.12. A game $\mathcal{G}_{2}$ with horizon $T=2$, discount factor $\delta \in(0,1]$ has two senders with payoff functions $f_{1}(\theta)=\theta^{0.3}$ and $f_{2}(\theta)=\theta^{2}$ and a receiver with payoff function $g(\theta)=\theta$. In the regular strategy profile, at period $T-1$ the thresholds are $\alpha_{1}^{T-1}=0.4171$ and $\alpha_{2}^{T-1}=0.5774$, hence the receiver obeys the sender 2 . Expected payoffs at period $T-1$ for the senders 1 and 2 respectively are 0.8367 and 0.4616. A game $\mathcal{G}_{3}$ has three senders with payoff functions $f_{1}(\theta)=\theta^{0.3}, f_{2}(\theta)=\theta^{2}$ and $f_{3}(\theta)=\theta^{0.5}$ and a receiver with payoff function $g(\theta)=\theta$. In the regular strategy profile, at period $T-1$ the thresholds are $\alpha_{1}^{T-1}=$ $0.4171, \alpha_{2}^{T-1}=0.5774$ and $\alpha_{3}^{T-1}=0.4444$, hence the receiver obeys the sender 3 . The expected payoffs at period $T-1$ for the senders 1 and 2 respectively are 0.8431 and 0.4522 . So, the sender 3 imposes positive externality for the sender 1 but negative externality for sender 2 . $\diamond$

\subsubsection{Externality of additional senders for the receiver}

Having additional senders means more information for the receiver. Intuitively it gives an impression that the receiver should be (at least weakly) better off in the game with fewer senders. However it tuns out that in the regular strategy profile, the receiver is not necessarily (even weakly) better off if there are fewer senders. Hence, the receiver is not always necessarily able to exploit the the additional information. 
Proposition 3.13. For every $n \geq 1$, there exist games $\mathcal{G}_{n}$ and $\mathcal{G}_{n+1}$, where $\mathcal{G}_{n+1}$ is a extension of $\mathcal{G}_{n}$, such that the sender $n+1$ imposes negative externality on the receiver.

The intuition for the fact that the expected payoff for the receiver could be strictly higher in game $\mathcal{G}_{n}$ than $\mathcal{G}_{n+1}$ is as follows. For simplicity assume $n=1$. Consider a scenario in which the receiver obeys the sender 2 at period $T-1$ in $\mathcal{G}_{2}$. At period $T-2$, the suggestion from sender 2 is highly undesirable for the receiver, so he obeys sender 1 . As suggestion of sender 1 at period $T-1$ is obeyed in $\mathcal{G}_{1}$ and not in $\mathcal{G}_{2}$, his thresholds at period $T-2$ are different. This may result in less expected payoff for the receiver at period $T-2$ in $\mathcal{G}_{2}$ than in $\mathcal{G}_{1}$.The example below illustrates this intuition and also serves as the proof of the Proposition 3.13 for $n=1$. Similar example can be constructed for any general $n$ by adding senders with same payoff functions.

Example 3.14. A game $\mathcal{G}_{1}$ has a sender with payoff function $f_{1}$ and a receiver with payoff function $g(\theta)=\theta$. A game $\mathcal{G}_{2}$ has two senders with payoff functions $f_{1}$ and $f_{2}(\theta)=\frac{1}{2}\left(1+(2 \theta-1)^{3}\right)$ and a receiver with payoff function $g(\theta)=\theta$. We fix the function $f_{1}$ later.

In game $\mathcal{G}_{2}$, in the regular strategy profile, the thresholds at periods $T-$ 1 are $\alpha_{1}^{T-1}=0.45$ and $\alpha_{2}^{T-1}=0.5$. Hence the receiver obeys the sender 2 at this period. As $\alpha_{1}^{T-2}=0.55$ and $\alpha_{2}^{T-2}=0.75$, the receiver obeys the sender 1 . However in game $\mathcal{G}_{1}$, in the regular strategy profile, the thresholds for the sender are $\alpha^{T-1}=0.45$ and $\alpha^{T-2}=0.62375$. The expected utility for the receiver at period $T-2$ is strictly greater in $\mathcal{G}_{1}$ than in $\mathcal{G}_{2}$.

The function $f_{1}$ is chosen such that the thresholds of the sender 1 in the games $\mathcal{G}_{1}$ and $\mathcal{G}_{2}$ are as above. It is thus sufficient to show that such function exists. Any strictly increasing function with following properties results in the required thresholds. 
$f(0.45)=\delta, f(0.5)=(1+30 e) \delta, f(0.55)=1.01 \delta, f(0.62375)=(1.01+$ $e) \delta, \int_{0}^{0.45} f(x) d x=(0.44-e) \delta, \int_{0.45}^{0.5} f(x) d x=(0.05+e) \delta, \int_{0}^{1} f(x) d x=$ $\delta$ where $\delta=\frac{1}{1.03}$ and $e$ is an infinitesimally small number.

It is an easy exercise to show that such functions exist and any function with these properties have the above mentioned thresholds.

\subsection{Concluding remarks}

This chapter shows that the model of sender-receiver stopping games with multiple senders differs significantly from the special case with only one sender. Unlike the setting with only 1 sender where there is a unique non-babbling PBE, the set of non-babbling PBEs here is quite rich.

In the multiple senders setting, the regular strategy profile is the extension of the unique non-babbling PBE from the 1 sender setting. It is an appealing feature that, the regular strategy profile does not allow manipulation by a coalition of senders.

Although the receiver makes better informed decisions with multiple senders, having an additional sender is not necessarily beneficial for the receiver, and not necessarily harmful for the existing senders. However there are PBEs in which the receiver can obtain the expected payoff which is maximum among all the strategy profiles, which was not true in the setting of only 1 sender.

\section{Appendix}

\section{Auxiliary lemmas}

For the statement and proofs in the appendix, we fix the strictly increasing continuous functions $f_{i}$ for $i \in N$ and $g$ from $I$ to $\mathbb{R}_{+}$such that $f_{i}(0)=0, g(0)=0$. 
Recall the function $H_{i}: I \rightarrow \mathbb{R}$ for each $i$ defined as $H_{i}(x)=f_{i}^{-1}(\delta \cdot[x$. $\left.\left.f_{i}(x)+\int_{x}^{1} f_{i}(\theta) d \theta\right]\right)$. For each $i$, define an auxiliary function $G_{i}: I \rightarrow \mathbb{R}$ is defined as $G_{i}(x)=x \cdot f_{i}(x)+\int_{x}^{1} f_{i}(\theta) d \theta$.

Lemma 3.15. For each $i$, the following statements hold:

[1] The functions $G_{i}$ and $H_{i}$ are strictly increasing,

[2] The function $H_{i}$ has a unique fixed point, denoted by $\zeta_{i}$,

[3] $H_{i}(y)>y$ for all $y<\zeta_{i}$ and $H_{i}(y)<y$ for all $y>\zeta_{i}$,

[4] $\zeta_{i} \rightarrow 1$ as $\delta \rightarrow 1$ and $\zeta_{i}=1$ when $\delta=1$.

Proof. [1] Take $0 \leq x<y \leq 1$. Because $f_{i}(x)<f_{i}(y)$, we have

$$
\begin{aligned}
G(y)-G(x) & =\left[y f_{i}(y)+\int_{y}^{1} f_{i}(\theta) d \theta\right]-\left[x f_{i}(x)+\int_{x}^{1} f_{i}(\theta) d \theta\right] \\
& =y f_{i}(y)-x f_{i}(x)-\int_{x}^{y} f_{i}(\theta) d \theta \\
& \geq(y-x) f_{i}(y)-\int_{x}^{y} f_{i}(\theta) d \theta>0 .
\end{aligned}
$$

Hence, $G_{i}$ is strictly increasing. The monotonicity of $H_{i}$ follows easily. This completes the proof of [1].

[2] We have $H_{i}(0)>0$ and $H_{i}(1) \leq 1$. As $H_{i}$ is strictly increasing, there exists $x \in I$ such that $H_{i}(x)=x$. Let $\zeta_{i}=\inf \left\{x \in I \mid H_{i}(x)=x\right\}$. By continuity of $H_{i}$, we have $H_{i}\left(\zeta_{i}\right)=\zeta_{i}$. Now we will prove part 3 of the lemma. This will imply that $\zeta_{i}$ is the unique solution of $H_{i}(x)=x$. This completes the proof of [2]. 
[3] We will show that the function $k_{i}(x)=f_{i}\left(H_{i}(x)\right)-f_{i}(x)$ is decreasing in $x \in I$. So, take $0 \leq x<y \leq 1$. We have

$$
\begin{aligned}
k_{i}(y)-k_{i}(x) & =\left[\delta \cdot G_{i}(y)-f_{i}(y)\right]-\left[\delta \cdot G_{i}(x)-f_{i}(x)\right] \\
& =\delta \cdot\left[G_{i}(y)-G_{i}(x)\right]-f_{i}(y)+f_{i}(x) \\
& =\delta \cdot\left[y f_{i}(y)-x f_{i}(x)-\int_{x}^{y} f(\theta) d \theta\right]-f_{i}(y)+f_{i}(x) \\
& =-(1-\delta y) \cdot f_{i}(y)+(1-\delta x) \cdot f_{i}(x)-\delta \cdot \int_{x}^{y} f_{i}(\theta) d \theta \\
& \leq-(1-\delta y) \cdot f_{i}(x)+(1-\delta x) \cdot f_{i}(x)-\delta \cdot \int_{x}^{y} f_{i}(\theta) d \theta \\
& =\delta \cdot\left[(y-x) \cdot f_{i}(x)-\int_{x}^{y} f_{i}(\theta) d \theta\right]<0 .
\end{aligned}
$$

So the function $k_{i}(x)=f_{i}\left(H_{i}(x)\right)-f_{i}(x)$ is indeed decreasing in $x \in I$.

Notice that $k\left(\zeta_{i}\right)=0$. Assume that $y<\zeta_{i}$. Then, since $k_{i}$ is decreasing, we have $k_{i}(y)>k_{i}\left(\zeta_{i}\right)=0$. Hence, $f_{i}\left(H_{i}(y)\right)>f_{i}(y)$, so $H_{i}(y)>y$. Similarly, if $y>\zeta_{i}$ then $H_{i}(y)<y$. This completes the proof of [3].

[4] As $\zeta_{i}$ is the unique fixed point of $H_{i}$, and for $\delta=1$ we have $H_{i}(1)=$ 1 , it follows that $\zeta_{i}=1$ when $\delta=1$. A continuity argument shows that $\zeta_{i} \rightarrow 1$ as $\delta \rightarrow 1$. This completes the proof of [4].

Recall that for each $i, \zeta_{i}^{T}=0$ and $\zeta_{i}^{t}=H_{i}\left(\zeta_{i}^{t+1}\right)$ for $t<T$ and the function $V$ is defined as $V(x)=\frac{1}{x} \cdot \int_{0}^{x} g(\theta) d \theta$. Note that for each $i$ and $t<T$, the number $\zeta_{i}^{t}$ depends on $\delta$. So, whenever necessary, we use the notation $\zeta_{i}^{t}(\delta)$. Recall that for each $i$ and $T>0, D_{i}^{T}$ is the smallest number in $[0,1]$ such that $\delta \cdot V(1) \geq V\left(\zeta_{i}^{1}(\delta)\right)$ for every $\delta \in\left(D_{i}^{T}, 1\right]$.

Lemma 3.16. The following statements hold:

[1] $1 \geq \zeta_{i}>\zeta_{i}^{1}>\zeta_{i}^{2}>\cdots>\zeta_{i}^{T}=0$. 
[2] $\frac{1}{\zeta_{i}^{t}} \cdot \int_{0}^{\zeta_{i}^{t}} g^{t}(\theta) d \theta<\int_{0}^{1} g^{t+1}(\theta) d \theta$ for $\delta \in\left[D_{i}^{T}, 1\right]$ and $t=1, \ldots, T-1$.

[3] $\zeta_{i}^{t}(\delta) \leq \zeta_{i}^{t}(1)$ for each $i, t \leq T$ and $\delta \in(0,1]$.

[4] $D_{i}^{T}<1$ for each $i$.

Proof. [1] By definition, $\zeta_{i}^{T}=0$. We also have

$$
\zeta_{i}^{T-1}=H_{i}\left(\zeta_{i}^{T}\right)=H_{i}(0)=f_{i}^{-1}\left(\delta \cdot \int_{0}^{1} f_{i}(\theta) d \theta\right)>0 .
$$

So, $\zeta_{i}^{T-1}>\zeta_{i}^{T}$. Then inductively $\zeta_{i}^{t}=H_{i}\left(\zeta_{i}^{t+1}\right)>H\left(\zeta_{i}^{t+2}\right)=\zeta_{i}^{t+1}$ for all $t=T-2, \ldots, 1$. Thus, $\zeta_{i}^{1}>\zeta_{i}^{2}>\cdots>\zeta_{i}^{T}=0$.

As $\zeta_{i}^{1}>\zeta_{i}^{2}$, we have $H_{i}\left(\zeta_{i}^{1}\right)>H_{i}\left(\zeta_{i}^{2}\right)=\zeta_{i}^{1}$. So, by lemma 3.15.3, we have $\zeta_{i}^{1}<\zeta_{i}$.

Finally, $\zeta_{i}=H_{i}\left(\zeta_{i}\right) \leq f_{i}^{-1}\left(\delta \cdot f_{i}(1)\right)<f_{i}^{-1}\left(f_{i}(1)\right)=1$, so $\zeta_{i}<1$. This completes the proof of [1].

[2] It holds that

$$
\frac{1}{\zeta_{i}^{t}} \cdot \int_{0}^{\zeta_{i}^{t}} g^{t}(\theta) d \theta<\frac{1}{\zeta_{i}^{1}} \cdot \int_{0}^{\zeta_{i}^{1}} g^{t}(\theta) d \theta \leq \delta \cdot \int_{0}^{1} g^{t}(\theta) d \theta=\int_{0}^{1} g^{t+1}(\theta) d \theta .
$$

The first inequality follows from from substituting $a=b=0, c=\zeta_{i}^{t}$, and $d=\zeta_{i}^{1}$ into Lemma 3.17. The second inequality follows from the fact that $\delta \cdot V(1) \geq V\left(\zeta_{i}^{1}\right)$, which is true due to the assumption $\delta \in$ $\left[D_{i}^{T}, 1\right]$. This completes the proof of [2].

[3] The proof is by backward induction on $t$. For $t=T$, we have $\zeta_{i}^{T}(\delta)=\zeta_{i}^{T}(1)=0$, so the statement is true. Assume the statement for $t+1$, so $\zeta_{i}^{t+1}(\delta) \leq \zeta_{i}^{t+1}(1)$ - We need to prove that $\zeta_{i}^{t}(\delta) \leq \zeta_{i}^{t}(1)$. Note that $\zeta_{i}^{t}(\delta)=H_{i}\left(\zeta_{i}^{t+1}(\delta)\right)=f_{i}^{-1}\left(\delta \cdot\left[\zeta_{i}^{t+1}(\delta) \cdot f_{i}\left(\zeta_{i}^{t+1}(\delta)\right)+\int_{\zeta_{i}^{t+1}(\delta)}^{1} f_{i}(\theta) d \theta\right]\right)$. 
For simplicity of notation, let $x=\zeta_{i}^{t+1}(\delta)$ and $y=\zeta_{i}^{t+1}(1)$. hence $x \leq y$. So, it is sufficient to show the following.

$$
\begin{aligned}
x \cdot f_{i}(x)+ & \int_{x}^{1} f_{i}(\theta) d \theta \leq y \cdot f_{i}(y)+\int_{y}^{1} f_{i}(\theta) d \theta \\
\int_{x}^{y} f_{i}(\theta) d \theta & \leq y \cdot f_{i}(y)-x \cdot f_{i}(x)
\end{aligned}
$$

LHS $\leq(y-x) \cdot\left(f_{i}(y)\right) \leq(y-x) \cdot\left(f_{i}(y)\right)+x \cdot(f(y)-f(x))=$ RHS. This completes the proof of [3].

[4] Recall that $V$ is a strictly increasing function. By [3], $\zeta_{i}^{1}(1) \geq \zeta_{i}^{1}(\delta)$ for $\delta \in(0,1]$. So, for each $\delta \geq \frac{V\left(\zeta_{i}^{1}(1)\right)}{V(1)}$, we have $\delta \cdot V(1) \geq V\left(\zeta_{i}^{1}(1)\right) \geq$ $V\left(\zeta_{i}^{1}(\delta)\right)$. Hence, $D_{i}^{T} \leq \frac{V\left(\zeta_{i}^{1}(1)\right)}{V(1)}$. By [1], we have $\zeta_{i}^{1}(1)<1$ and so, $\frac{V\left(\zeta_{i}^{1}(1)\right.}{V(1)}<1$. This implies that $D_{i}^{T}<1$.

Lemma 3.17. Let $g: \mathbb{R} \rightarrow \mathbb{R}$ be a non-decreasing function. Then, for any $a \leq b<c \leq d$,

$$
\frac{1}{c-a} \int_{a}^{c} g(x) d x \leq \frac{1}{d-b} \int_{b}^{d} g(x) d x .
$$

The inequality is strict if $g$ is strictly increasing.

Proof. Define $\eta:[a, c] \rightarrow[b, d]$ by

$$
\eta(x)=\frac{d-b}{c-a} \cdot x+\frac{b c-a d}{c-a} .
$$

Then $\eta(a)=b$ and $\eta(c)=d$, and $\eta$ is a linear bijection between $[a, c]$ and $[b, d]$. Because $a \leq b$ and $c \leq d$ and $\eta$ is linear, we have $x \leq \eta(x)$ for all $x \in[a, c]$. 
Write $h(x)=(g \circ \eta)(x)$ for all $x \in[a, c]$. Since $x \leq \eta(x)$ and $g$ is nondecreasing, $g \leq h$ on $[a, c]$. By using substitution, it follows that

$$
\begin{aligned}
\frac{1}{c-a} \int_{a}^{c} g(x) d x & \leq \frac{1}{c-a} \int_{a}^{c} h(x) d x \\
& =\frac{1}{d-b} \int_{a}^{c}(g \circ \eta)(x) d \eta(x) \\
& =\frac{1}{d-b} \int_{b}^{d} g(y) d y .
\end{aligned}
$$

This completes the proof. 



\section{4}

Group strategy-proof rules in multidimensional binary domains 


\subsection{Introduction}

We consider ${ }^{1}$ social decision making problems with $n$ agents, each of who has an opinion (zero or one) on each of $m$ issues, to be called components. Any $m$-vector of zeros and ones is called an alternative, and the goal is to find a social, compromise alternative. The preference of an agent for an alternative is determined by the (Hamming) distance of that alternative to the agent's top alternative: the smaller that distance is, the better. Thus, an agent's preference is completely determined by that agent's top alternative, and each agent is asked to report this. A social choice function assigns to each profile of reported alternatives a (social) alternative. We assume, almost throughout, that a social choice function is unanimous (if all reported alternatives are equal then that common alternative should be assigned), anonymous (only the reported alternatives matter and not who reports what), and neutral (components are treated equally: renaming the components in the preference profile results in renaming the components in the outcome in the same way). Such a social choice function will simply be called a rule. We will be interested in strategy-proofness (no agent can be better off by not reporting the top alternative), weak group strategyproofness (no group of agents can make each of its members better off by not reporting truthfully), and strong group strategy-proofness (no group of agents can make each of its members at least as well and at least one member better off by not reporting truthfully). Of course, this model fits within a large literature, but we will first describe our main results and next relate our model and results to the literature.

In this context, it turns out that strategy-proofness is a fairly weak requirement. We show that component-wise majority rules are strategyproof. A component-wise majority rule simply assigns to each component a zero if the majority of agents assigns a zero to that component, and a one if the majority of agents assigns a one, with a tie-breaker in

\footnotetext{
${ }^{1}$ This chapter is based on joint work with Hans Peters. I would like to thank Ulle Endriss and Zoi Terzopoulou for their very helpful comments. I would also like to thank Arunava Sen and Swaprava Nath for helpful discussions on a preliminary note on which this paper is based.
} 
the case that the number of agents is even. For the case of two agents and two components, we describe all (six) strategy-proof rules; this is a rather elaborate task, and we learn from it that, without additional conditions, characterizing all strategy-proof rules for the general case will be practically infeasible. We next turn to weak group strategyproofness. Somewhat surprisingly, it turns out that for at least four agents and at least three components, component-wise majority rules are not weakly group strategy-proof. This, however, does not mean that there are no weakly group strategy-proof rules, and for three components and two or more agents we exhibit an example of such a rule. Finally, we turn to strong group strategy-proofness. Here, we show that for at least four agents and at least three components, a strong group strategy-proof rule has a so-called dominant alternative: this is an alternative, necessarily with all components zero or all components one due to our conditions on a rule, that is always chosen if reported by at least one agent. In turn, we show that this actually results in an impossibility: for at least four agents and at least three components, there exists no strong group strategy-proof rule.

Our model is a special case of the classical social choice model. If all preferences were allowed then the well-known result of Gibbard [16] and Satterthwaite [33] would imply that there is no strategy-proof rule - recall that a rule is unanimous and anonymous by definition. Of course, our domain of preferences is very small, and from this aspect it is not a surprise that there do exist strategy-proof rules. Nevertheless, similar models in the literature do exhibit non-existence of such rules. Observe that in our model, alternatives can be seen as the vertices in a hypercube (a square if there are two components, a cube if there are three, etc.). Preference is then simply determined by the Euclidean distance between the vertex corresponding to the top alternative and any other vertex, measured along the edges of the hypercube. This model closely resembles the model in Schummer and Vohra [34] for more general graphs, but there every point of each edge is an alternative (thus, the number of alternatives is infinite). This difference appears to be essential: they find that there exists no strategy-proof rule, in contrast to 
our results. The model is also a special case of the model in Peters, Roy, and Sadhukan [30], but they consider a different class of preferences, namely all preferences that are single-peaked with respect to a spanning tree of the graph: for instance, in the case of two components, a preference $11 \succ 10 \succ 00 \succ 01$ would be allowed, but in our model, if the top alternative is 11, then 01 is (always) preferred to 00. Again, this difference appears to be essential, since also [30] find that there exists no strategy-proof rule.

Another strand of literature to which our model is related, is that of judgment aggregation: a rule aggregates the judgments of the agents over a number of different issues to a common judgment over these issues (without there being a conclusion as in the classical judgement aggregation problem). Terzopoulou and Endriss [38] assume a larger class of preferences and show (their Theorem 1) that a rule is strategyproof if and only if it is independent (the value assigned to each component depends only on the agents' values assigned to that component and not on the values they assign to any other component) and monotonic (in the values per component). A typical example of such a rule is component-wise majority. Our class of preferences is smaller and, indeed, we show that other strategy-proof rules exist: see our Example 4.13, which exhibits a strategy-proof rule which is not independent. Botan, Novaro, and Endriss [7] consider the same preferences as we do, and show that an unbiased rule (i.e. flipping zeros and ones in a component leads to a corresponding flip (only) in the same component of the social alternative) is strategy-proof if and only if it is independent and monotonic (their Theorem 4). Our rule in Example 4.13 is, indeed, not unbiased. Dietrich and List [10] introduced strategy-proofness (non-manipulability) in the judgement aggregation model but impose additional conditions (compared to [38]) on a rule, resulting in an impossibility (their Theorem 2). [7] also consider manipulation by groups, but mainly focus on unbiased rules, as explained above.

Formally, our model also resembles approval voting (Brams and Fishburn, [8]). Components can be interpreted as candidates, and reported 
alternatives as approval votes: one means approval, zero means disapproval. The alternative assigned by a rule then gives the approved candidates. An important difference is that underlying approval voting are preferences of agents over candidates, which are absent in our model.

The model in this chapter is also studied in computer science, e.g., under the name (and application) of classification: the alternatives correspond to examples described by binary labels, and a rule corresponds to a classifier, aimed at finding an alternative that best matches the reported examples. See, e.g., Meir, Procaccia, and Rosenschein [27].

The chapter is organized as follows. Section 4.2 introduces the model and a few preliminary results. Sections $4.3,4.4$, and 4.5 consider strategy-proofness, weak group strategy-proofness, and strong group strategy-proofness, respectively. Section 4.6 concludes.

\subsection{Model and preliminary results}

\subsubsection{Model and definitions}

For $m, n \in \mathbb{N}$ with $m, n \geq 2$, the set of components is $M=\{1, \ldots, m\}$, the set of agents is $N=\{1, \ldots, n\}$, and the set of alternatives is $A=$ $\{0,1\}^{M}$. For $a=\left(a_{1}, a_{2}, \ldots, a_{m}\right) \in A, a_{j} \in\{0,1\}$ is the value of the $j^{\text {th }}$ component of $a$.

For all $a, b \in A$ let $r(a, b)=\sum_{j=1}^{m}\left|a_{j}-b_{j}\right|$, i.e., $r(a, b)$ is the Hamming distance between $a$ and $b$.

A preference of an agent $i$ on $A$ is characterized by its unique top alternative $a^{i} \in A$ : an alternative $b$ is (weakly) preferred by agent $i$ to alternative $c$ if and only if $r\left(b, a^{i}\right) \leq r\left(c, a^{i}\right)$. The set of (preference) profiles is denoted by $A^{N}$. We use the notation $a^{N}$ for the profile $\left(a^{1}, \ldots, a^{n}\right)$, and for $S \subseteq N$ we denote by $a^{S} b^{N-S}$ the profile $\left(c^{1}, \ldots, c^{n}\right) \in A^{N}$ with $c^{i}=a^{i}$ for all $i \in S$ and $c^{i}=b^{i}$ for all $i \in N-S$. Similar notations will 

domains

be clear from the context. In general, we use superscripts for agents and subscripts for components.

A social choice function is a map $f: A^{N} \rightarrow A$.

A social choice function $f$ is unanimous if $f(a, \ldots, a)=a$ for all $a \in A$.

Two profiles $a^{N}$ and $b^{N}$ are similar if there is a permutation $\pi$ of $N$ such that $a^{i}=b^{\pi(i)}$ for every $i \in N$. A social choice function $f$ is anonymous if $f\left(a^{N}\right)=f\left(b^{N}\right)$ for all similar profiles $a^{N}$ and $b^{N}$.

For $a \in A$ and a permutation $\pi$ of $M$ we denote by $\pi a \in A$ the alternative with $(\pi a)_{j}=a_{\pi(j)}$ for every $j \in M$. A social choice function $f$ is neutral if $f\left(\pi a^{1}, \ldots, \pi a^{n}\right)=\pi f\left(a^{N}\right)$ for every $a^{N} \in A^{N}$ and every permutation $\pi$ of $M$.

A social choice function $f$ has a dominant alternative $d \in A$ if $f\left(a^{N}\right)=d$ whenever $a^{i}=d$ for some $i \in N$. Clearly, if such a dominant alternative exists, it is unique.

A social choice function $f$ is weakly Pareto optimal if for every profile $a^{N} \in A^{N}$, there does not exist an alternative $b \in A$ such that $r\left(b, a^{i}\right)<r\left(f\left(a^{N}\right), a^{i}\right)$ for all $i \in N$. A social choice function $f$ is strongly Pareto optimal if for every profile $a^{N} \in A^{N}$, there does not exist an alternative $b \in A$ such that $r\left(b, a^{i}\right) \leq r\left(f\left(a^{N}\right), a^{i}\right)$ for all $i \in N$, with at least one inequality strict. A social choice function $f$ is componentwise unanimous if for each $j \in M$ and $x \in\{0,1\}, a_{j}^{i}=x$ for each agent $i$ implies $f\left(a^{N}\right)_{j}=x$. Clearly, if $f$ is weakly Pareto optimal, then it is also component-wise unanimous.

In this chapter, the conditions of central interest for a social choice function $f$ are the following.

Definition 4.1. An agent $i$ can manipulate $f$ at profile $a^{N}$ if there is a $b^{i} \in A$ such that $r\left(f\left(b^{i}, a^{N-\{i\}}\right), a^{i}\right)<r\left(f\left(a^{N}\right), a^{i}\right)$; $f$ is strategy-proof if at every profile there is no agent who can manipulate $f$. 
Definition 4.2. A subset $S \subseteq N$ can strongly manipulate $f$ at profile $a^{N}$ if there are $b^{i} \in A, i \in S$, such that $r\left(f\left(b^{S}, a^{N-S}\right), a^{i}\right)<r\left(f\left(a^{N}\right), a^{i}\right)$ for every $i \in S$; $f$ is weakly group strategy-proof if at every profile there is no subset of agents which can strongly manipulate $f$.

Definition 4.3. A subset $S \subseteq N$ can weakly manipulate $f$ at profile $a^{N}$ if there are $b^{i} \in A, i \in S$, such that $r\left(f\left(b^{S}, a^{N-S}\right), a^{i}\right) \leq r\left(f\left(a^{N}\right), a^{i}\right)$ for every $i \in S$, with at least one inequality strict; $f$ is strongly group strategy-proof if at every profile there is no subset of agents which can weakly manipulate $f$.

Throughout the rest of this chapter we consider only social choice functions that are unanimous, anonymous, and neutral.

Definition 4.4. A rule is a unanimous, anonymous, and neutral social choice function.

\subsubsection{Further notations}

In this subsection we define some notations in order to efficiently represent profiles.

For an alternative $a=\left(a_{1}, \ldots, a_{m}\right)$ (or a preference with $a$ as its top alternative), we also use the notation $a_{1} \ldots a_{m}$. We denote by $0_{x_{1}} 1_{y_{1}} 0_{x_{2}} 1_{y_{2}} \ldots 0_{x_{k}} 1_{y_{k}}$ the alternative containing $x_{1}$ zeros followed by $y_{1}$ ones followed by $x_{2}$ zeros, and so on. If a subscript $x_{j}$ or $y_{j}$ is equal to 1 , we leave it out: for example, $010_{m-2}$ starts with a zero, followed by a one, which is followed by $m-2$ zeros.

Since we only consider anonymous social choice functions, the identities of the agents do not matter. We use the notation $\left(a^{\left[z_{1}\right]}, b^{\left[z_{2}\right]}, \ldots\right)$ for the profile in which $z_{1}$ agents have preference $a, z_{2}$ agents have preference $b$, etc. 


\subsubsection{Preliminary results}

We say that a profile $b^{N}$ is obtained from $a^{N}$ by swapping the components $j$ and $k$ if for all $i \in N$ we have $b^{i}=\pi a^{i}$, where $\pi$ is the permutation of $M$ with $\pi(j)=k, \pi(k)=j$, and $\pi(r)=r$ otherwise. A profile $a^{N}$ is symmetric in components $j$ and $k$ if $a^{N}$ is similar to the profile $b^{N}$ obtained by swapping the components $j$ and $k$.

Lemma 4.5. Let $f$ be a rule, and let profile $a^{N}$ be symmetric in components $j$ and $k$. Then $f\left(a^{N}\right)_{j}=f\left(a^{N}\right)_{k}$.

Proof. Profile $a^{N}$ is similar to the profile $b^{N}$ obtained from $a^{N}$ by swapping the components $j$ and $k$. By neutrality of $f$, we have $f\left(a^{N}\right)_{j}=$ $f\left(b^{N}\right)_{k}$. Anonymity of $f$ implies $f\left(a^{N}\right)=f\left(b^{N}\right)$. Hence, $f\left(a^{N}\right)_{j}=$ $f\left(b^{N}\right)_{k}=f\left(a^{N}\right)_{k}$.

Lemma 4.6. Let $f$ have a dominant alternative $a$. Then $a=0_{m}$ or $a=1_{m}$.

Proof. Suppose, contrary to what we wish to prove, that $a_{j} \neq a_{k}$ for some $j, k \in M$. Let $a^{\prime} \in A$ with $a_{j}^{\prime}=a_{k}, a_{k}^{\prime}=a_{j}$, and $a_{l}^{\prime}=a_{l}$ otherwise. Consider the profiles $V=\left(a, a^{\prime}, 0_{m}^{N-\{1,2\}}\right)$ and $V^{\prime}=\left(a^{\prime}, a, 0_{m}^{N-\{1,2\}}\right)$. Then $f(V)=f\left(V^{\prime}\right)=a$ by anonymity. On the other hand, neutrality of $f$ and $f(V)=a$ imply $f\left(V^{\prime}\right)=a^{\prime}$. Since $a \neq a^{\prime}$, this is a contradiction.

\subsection{Strategy-proofness}

We start with establishing a necessary condition - useful later on in the chapter - for a rule to be strategy-proof. Next, we show that component-wise majority rules are strategy-proof, and we determine all strategy-proof rules in the case of two agents and two components. One thing we learn from this is that, in general, it is practically infeasible to find all strategy-proof rules. We conclude this 
section by exhibiting an example of a strategy-proof rule that is not a component-wise majority rule, and has a dominant alternative.

\subsubsection{Switching points}

A switching point of a rule $f$ is an integer $n_{0}$ with $1 \leq n_{0} \leq n$ such that

$$
f\left(0_{m}^{\left[n^{\prime}\right]}, 1_{m}^{\left[n-n^{\prime}\right]}\right)= \begin{cases}0_{m} & \text { if } n^{\prime} \geq n_{0} \\ 1_{m} & \text { if } n^{\prime}<n_{0} .\end{cases}
$$

Clearly, if a switching point exists then it is unique.

The following lemma shows that strategy-proofness implies the existence of a switching point.

Lemma 4.7. Let $f$ be strategy-proof rule. Then $f$ has a switching point $n_{0}$. Further, if $f$ does not have a dominant alternative, then $2 \leq n_{0} \leq n-1$.

Proof. For $l \in\{0,1, \ldots, n\}$, denote by $V^{l}$ the profile $\left(0_{m}^{[l]}, 1_{m}^{[n-l]}\right)$. Observe that $V^{l}$ is symmetric in any two components. Thus, Lemma 4.5 implies that $f\left(V^{l}\right)_{j}=f\left(V^{l}\right)_{j^{\prime}}$ for any $j, j^{\prime} \in M$. Hence, for every $l \in\{0,1, \ldots, n\}, f\left(V^{l}\right)=0_{m}$ or $f\left(V^{l}\right)=1_{m}$.

Let $n_{0}=\min \left\{l \in\{0,1, \ldots, n\} \mid f\left(V^{l}\right)=0_{m}\right\}$. By unanimity, $f\left(V^{0}\right)=$ $1_{m}$ and $f\left(V^{n}\right)=0_{m}$. Thus, $1 \leq n_{0} \leq n$.

If $f\left(V^{n_{0}+1}\right)=1_{m}$, an agent with preference $0_{m}$ in $V^{n_{0}+1}$ can report $1_{m}$ so that the alternative $f\left(V^{n_{0}}\right)=0_{m}$ results, contradicting strategyproofness of $f$. Hence, $f\left(V^{n_{0}+1}\right)=0_{m}$. Iterating this argument, we obtain $f\left(V^{l}\right)=0_{m}$ for each $n_{0} \leq l \leq n$. Hence, $f\left(V^{l}\right)=0_{m}$ if $l \geq n_{0}$ and $f\left(V^{l}\right)=1_{m}$ if $l<n_{0}$. Therefore, $n_{0}$ is a switching point.

Suppose, additionally, that $f$ has no dominant alternative. We show that $2 \leq n_{0} \leq n-1$. By way of contradiction, suppose $n_{0}=1$. Hence, $f\left(V^{1}\right)=0_{m}$. Since $0_{m}$ is not a dominant alternative of $f$, there exists a 
profile $\left(0_{m}, a^{[n-1]}\right)$ such that $f\left(0_{m}, a^{[n-1]}\right) \neq 0_{m}$. Consider a sequence of profiles $R^{1}=\left(0_{m}, 1_{m}^{[n-1]}\right)$ and $R^{i}=\left(0_{m}, a^{2}, \ldots, a^{i}, 1_{m}^{[n-i]}\right)$ for $i=$ $2, \ldots, n$. Since $f\left(R^{1}\right)=f\left(V^{1}\right)=0_{m}$ and $f\left(R^{n}\right)=f\left(0_{m}, a^{[n-1]}\right) \neq 0_{m}$ ， there exists $i$ such that $f\left(R^{i}\right)=0_{m}$ and $f\left(R^{i+1}\right) \neq 0_{m}$. Then agent $i+1$ in profile $R^{i}$, who has top alternative $1_{m}$, can strictly benefit from reporting $a^{i+1}$, thus obtaining $f\left(R^{i+1}\right) \neq 0_{m}$. This contradicts strategyproofness of $f$. Therefore, $2 \leq n_{0}$. By a similar argument it follows that $n_{0} \leq n-1$. This completes the proof of the lemma.

\subsubsection{Component-wise majority rules}

A natural rule is to assign to each profile an alternative of which each component is the majority of the respective components in the preferences of the agents. The formal definition is as follows.

Definition 4.8. A social choice function $f$ is a component-wise majority rule with tie breaker $t \in\left\{0_{m}, 1_{m}\right\}$ if for every profile $a^{N} \in A^{N}$ and every $j \in M$, if $\left|\left\{i \in N \mid a_{j}^{i}=1\right\}\right|>\frac{n}{2}$ then $f\left(a^{N}\right)_{j}=1$, if $\mid\left\{i \in N \mid a_{j}^{i}=\right.$ $0\} \mid>\frac{n}{2}$ then $f\left(a^{N}\right)_{j}=0$, and if $\left|\left\{i \in N \mid a_{j}^{i}=0\right\}\right|=\left|\left\{i \in N \mid a_{j}^{i}=1\right\}\right|$, then $f\left(a^{N}\right)_{j}=t_{j}$.

Clearly, a component-wise majority rule is unanimous, anonymous, and neutral and thus, indeed, a rule. Also, if there are at least three agents, a component-wise majority rule does not have a dominant alternative. In the case of two agents a component-wise majority rule has dominant alternative $0_{m}$ if $t=0_{m}$ and $1_{m}$ if $t=1_{m}$.

The tie breaker $t$ is only relevant when the number of agents is even. The following proposition considers strategy-proofness of componentwise majority rules.

Proposition 4.9. Each component-wise majority rule is strategy-proof. 
Proof. Let $f$ be a component-wise majority rule with tie breaker $t$. Suppose that $f$ is not strategy-proof. Then there exists a profile $a^{N}$, an agent $i$, and a preference $b^{i}$ for agent $i$ such that $r\left(f\left(b^{i}, a^{N-\{i\}}\right), a^{i}\right)<$ $r\left(f\left(a^{N}\right), a^{i}\right)$. Writing $a=f\left(a^{N}\right)$ and $b=f\left(b^{i}, a^{N-\{i\}}\right)$, this implies that there is a component $j \in M$ such that $b_{j}=a_{j}^{i} \neq a_{j}$. Without loss of generality we may assume that $a_{j}^{i}=b_{j}=1$ and $a_{j}=0$. Since $a_{j}=f\left(a^{N}\right)_{j}=0$, either strictly more than $\frac{n}{2}$ preferences in the profile $a^{N}$ have 0 at component $j$ or exactly $\frac{n}{2}$ preferences in the profile $a^{N}$ have 0 at component $j$ and $t_{j}=0$. Since $a_{j}^{i}=1$ and each agent other than $i$ has the same preference in the profiles $a^{N}$ and $\left(b^{i}, a^{N-\{i\}}\right)$, we have $b_{j}=0$, which is a contradiction.

Proposition 4.9 would still hold for general tie breakers $t \in A$, different from $0_{m}$ or $1_{m}$, but then neutrality is violated. It does not necessarily hold if ties are broken in different ways. We illustrate this by the following example.

Example 4.10. Let $m=3$ and $n=4$. For any preference profile and any component let the rule $f$ assign 0 (1) if there is a strict majority for 0 (1). In case of a tie at some component, let $f$ assign 1 if the total number of $1 \mathrm{~s}$ in the profile strictly exceeds the total number of $0 \mathrm{~s}$; otherwise, let $f$ assign 0 . Then $f$ is unanimous, anonymous, and neutral, and it respects component-wise majority without being a component-wise majority rule in the sense of Definition 4.8. Observe that $f(010,010,110,111)=110$ and $f(000,010,110,111)=010$. Since agent 1 with preference 010 prefers 010 over 110, this implies that $f$ is not strategy-proof.

Another observation about component-wise majority rules is the following.

Proposition 4.11. Let $f$ be a component-wise majority rule. Then $f$ is strongly Pareto optimal. 

domains

Proof. Observe that $f$ minimizes total Hamming distance, that is, for every profile $a^{N}$ and every $b \in A$ we have

$$
\sum_{i \in N} r\left(f\left(a^{N}\right), a^{i}\right) \leq \sum_{i \in N} r\left(b, a^{i}\right) .
$$

Hence, there is no $b \in A$ with $r\left(b, a^{i}\right) \leq r\left(f\left(a^{N}\right), a^{i}\right)$ for all $i \in N$ such that at least one of these inequalities is strict. This implies that $f$ is strongly Pareto optimal.

As is easy to see from its proof, Proposition 4.11 would still hold if ties are broken in arbitrary ways.

A simple consequence of Propositions 4.9 and 4.11 is that for two agents any component-wise majority rule is strongly (and hence also weakly) group strategy-proof (see Sections 4.4 and 4.5 for further consideration of weak and strong group strategy-proofness, respectively).

\subsubsection{Two agents and two components}

In this subsection, we discuss the simple case when there are only two agents $(n=2)$ and two components $(m=2)$.

Proposition 4.12. Let $n=m=2$. There are exactly six strategy-proof rules, two of which are the component-wise majority rules. All these rules have a dominant alternative.

Proof. Let $f$ be a strategy-proof rule. To completely specify $f$, because of unanimity, anonymity, and neutrality, we only need to fix the alternatives that $f$ assigns to four profiles: $(00,01),(00,11),(01,10)$, and $(01,11)$. The profile $(00,11)$ is symmetric in the components 1 and 2 , so by Lemma $4.5, f(00,11)_{1}=f(00,11)_{2}$. Thus, $f(00,11)=00$ or $f(00,11)=11$. 
Consider the case where $f(00,11)=00$. Then $f(00,01)=00$, otherwise the agent with preference 11 in the profile $(00,11)$ can report 01 and get a better outcome, contradicting strategy-proofness. Then also $f(00,10)=00$ due to neutrality and $f(00,00)=00$ due to unanimity . Hence, 00 is a dominant alternative. The profile $(01,10)$ is symmetric in components 1 and 2 , so $f(01,10)=00$ or $f(01,10)=11$. We now consider the profile $(01,11)$. If $f(01,11)=10$, then the agent with preference 01 in the profile $(01,11)$ can report 11 and get a better outcome. Similarly if $f(01,11)=00$, the agent with preference 11 can misreport to 01 . Hence, $f(01,11)=01$ or $f(01,11)=11$. Moreover, if $f(01,10)=11$ then also $f(01,11)=11$, otherwise the agent with preference 11 in the profile $(01,11)$ can misreport 10 to get the outcome 11 . Hence, there are exactly three unanimous, anonymous, neutral and strategy-proof social choice functions when $f(00,11)=00$. By symmetry, there are also three unanimous, anonymous, neutral, and strategy-proof SCFs when $f(00,11)=11$, and these have 11 as dominant alternative.

Thus, there are exactly six strategy-proof rules, and all have a dominant alternative. Two of these rules are the component-wise majority rules.

The rules derived in Proposition 4.12 are displayed in Table 4.3.3.

\subsubsection{Further results on strategy-proofness}

For $m=3$ we exhibit an example of a rule which is strategy-proof and has a dominant alternative.

Example 4.13. The rule $f$ is defined as follows. Let $m=3$ and let $a^{N}$ be a preference profile.

(i) If $a^{i}=b$ for every $i \in N$ and some $b \in A$, then $f\left(a^{N}\right)=b$.

(ii) Otherwise, if $a^{i} \in\{011,101,110,111\}$ for every $i \in N$, then $f\left(a^{N}\right)=111$. 


\begin{tabular}{||c|c||l|l|l||l|l|l||}
\hline \hline \multicolumn{2}{||c|}{ profile } & $f^{1}$ & $f^{2}$ & $f^{3}$ & $f^{4}$ & $f^{5}$ & $f^{6}$ \\
\hline 00 & 00 & 00 & 00 & 00 & 00 & 00 & 00 \\
00 & 01 & 00 & 00 & 00 & 00 & 00 & 01 \\
00 & 10 & 00 & 00 & 00 & 00 & 00 & 10 \\
00 & 11 & 00 & 00 & 00 & 11 & 11 & 11 \\
01 & 00 & 00 & 00 & 00 & 00 & 00 & 01 \\
01 & 01 & 01 & 01 & 01 & 01 & 01 & 01 \\
01 & 10 & 00 & 11 & 00 & 00 & 11 & 11 \\
01 & 11 & 01 & 11 & 11 & 11 & 11 & 11 \\
10 & 00 & 00 & 00 & 00 & 00 & 01 & 10 \\
10 & 01 & 00 & 11 & 00 & 00 & 11 & 11 \\
10 & 10 & 10 & 10 & 10 & 10 & 10 & 10 \\
10 & 11 & 10 & 11 & 11 & 11 & 11 & 11 \\
11 & 00 & 00 & 00 & 00 & 11 & 11 & 11 \\
11 & 01 & 01 & 11 & 11 & 11 & 11 & 11 \\
11 & 10 & 10 & 11 & 11 & 11 & 11 & 11 \\
11 & 11 & 11 & 11 & 11 & 11 & 11 & 11 \\
\hline
\end{tabular}

Table 1. The six strategy-proof rules if $m=n=2$. Rules $f^{1}, f^{2}$, and $f^{3}$ have dominant alternative 00 , the other rules have dominant alternative 11 . Rules $f^{1}$ and $f^{6}$ are the component-wise majority rules with $t=00$ and $t=11$, respectively. 
(iii) Otherwise, if $a^{k}=001$ for some $k \in N$ and $a_{3}^{i}=1$ for all $i \in N$, then $f\left(a^{N}\right)=001$.

(iv) In all remaining cases, $f\left(a^{N}\right)=000$.

We leave it to the reader to verify that $f$ is indeed a rule and that it is strategy-proof. Clearly, 000 is a dominant alternative. Also, e.g. $f(011, \ldots, 011,101)=111$, hence if $n \geq 3$ then at component 1 there is a strict majority for 0 , whereas $f$ assigns 1 .

\subsection{Weak group strategy-proofness}

In this section we strengthen strategy-proofness to weak group strategy-proofness. We first establish some useful results concerning weakly group strategy-proof rules. Next, we consider again component-wise majority rules.

We first observe that weak group strategy-proofness implies weak Pareto optimality.

Lemma 4.14. Let $f$ be a weakly group strategy-proof rule. Then $f$ is weakly Pareto optimal.

Proof. By way of contradiction suppose there exist a profile $a^{N}$ and an alternative $b$ such that for each agent $i \in N, r\left(b, a^{i}\right)<r\left(f\left(a^{N}\right), a^{i}\right)$. By unanimity, $f\left(b^{[n]}\right)=b$, hence $N$ can strongly manipulate at $a^{N}$. This contradicts weak group strategy-proofness of $f$.

Now we turn again to component-wise majority rules. ${ }^{2}$ We have already seen that for the case of two agents, both component-wise majority rules are weakly group strategy-proof - this follows from Propositions 4.9 and 4.11 .

\footnotetext{
${ }^{2}$ Recall that, by definition, there are two component-wise majority rules if $n$ is even, and only one if $n$ is odd.
} 
Chapter 4. Group strategy-proof rules in multidimensional binary domains

Proposition 4.15. Let $n=3$. Then for every $m \geq 2$ the component-wise majority rule is weakly group strategy-proof.

Proof. Let $m \geq 2$ and let $f$ be the component-wise majority rule. In view of Propositions 4.9 and 4.11, only two-agent coalitions could possibly strongly manipulate. Consider, without loss of generality $\{1,2\} \subseteq N$. At any preference profile and any component $j$, if $\{1,2\}$ misreports in order to change the outcome, then either both agents are worse off at that component (namely if they both have 0 or both 1 at $j$ ), or one agent is better off while the other is worse off (namely, if one has 0 and the other has 1 at $j$ ). This implies that $\{1,2\}$ cannot strongly manipulate.

Proposition 4.16. Let $m=2$. Then for every $n \geq 2$ each component-wise majority rule is weakly group strategy-proof.

Proof. Let $a^{N}$ be a preference profile and without loss of generality assume that $f\left(a^{N}\right)=00$. Let $S \subseteq N$ and $b^{S} \in A^{S}$. If $f\left(b^{S}, a^{N-S}\right)=10$, then $S$ strongly manipulates if $a^{i} \in\{10,11\}$ for all $i \in S$, but then

$$
\left|\left\{i \in N \mid a_{1}^{i}=1\right\}\right| \geq\left|\left\{i \in S \mid b_{1}^{i}=1\right\}\right|+\left|\left\{i \in N-S \mid a_{1}^{i}=1\right\}\right|
$$

so that $f_{1}\left(b^{S}, a^{N-S}\right)=0$, a contradiction. The case where $f\left(b^{S}, a^{N-S}\right)=01$ is similar. If $f\left(b^{S}, a^{N-S}\right)=11$, then $S$ strongly manipulates if $a^{i}=11$ for all $i \in S$, and then

$$
\left|\left\{i \in N \mid a^{i}=11\right\}\right| \geq\left|\left\{i \in S \mid b^{i}=11\right\}\right|+\left|\left\{i \in N-S \mid a^{i}=11\right\}\right|
$$

so that $f\left(b^{S}, a^{N-S}\right)=00$, a contradiction. Hence $f$ is weakly group strategy-proof.

Proposition 4.17. Let $n \geq 4$ and $m \geq 3$. Then no component-wise majority rule is weakly group strategy-proof. 
Proof. We first consider the case where $n$ is odd. By way of contradiction suppose that the component-wise majority rule $f$ is weakly group strategy-proof.

Consider the profile $V=\left(0_{m-3} 001,0_{m-3} 010,0_{m-3} 100,0_{m}^{\left[\frac{n-5}{2}\right]}, 1_{m}^{\left[\frac{n-1}{2}\right]}\right)$. Then $f(V)=0_{m-3} 1_{3}$. The disutility for the first three agents is 2 . If these three agents form a coalition and all report $0_{m}$, the new profile is $V^{\prime}=\left(0_{m}^{\left[\frac{n+1}{2}\right]}, 1_{m}^{\left[\frac{n-1}{2}\right]}\right)$, and $f\left(V^{\prime}\right)=0_{m}$. Hence the disutility for the first three agents of the new alternative is 1 , which is strictly less. This is a contradiction to the assumption that $f$ is weakly group strategy-proof.

Now we consider the case where $n$ is even. First assume $n \geq 6$, and let $f$ be the component-wise majority rule with tie breaker $t$. Let $t^{\prime}=$ $1_{m}-t$. Consider the profile

$$
\begin{gathered}
V=\left(t_{1}^{\prime} t_{2} t_{3} 0_{m-3}, t_{1} t_{2}^{\prime} t_{3} 0_{m-3}, t_{1} t_{2} t_{3}^{\prime} 0_{m-3}, t_{1} t_{2}^{\prime} t_{3}^{\prime} 0_{m-3}\right. \\
\left.t_{1}^{\prime} t_{2} t_{3}^{\prime} 0_{m-3}, t_{1}^{\prime} t_{2}^{\prime} t_{3} 0_{m-3}, 0_{m}^{\left[\frac{n-6}{2}\right]}, 1_{m}^{\left[\frac{n-6}{2}\right]}\right) .
\end{gathered}
$$

In this profile, the components $1,2,3$ have exactly $\frac{n}{2}$ zeros and the components $4, \ldots, m$ strictly more than $\frac{n}{2}$ zeros. Since $f$ is a componentwise majority rule with tie breaker $t, f(V)=t_{1} t_{2} t_{3} 0_{m-3}$. This alternative has disutility 2 for agents $4,5,6$; if these agents all report $t_{1}^{\prime} t_{2}^{\prime} t_{3}^{\prime} 0_{m-3}$, the new outcome is $t_{1}^{\prime} t_{2}^{\prime} t_{3}^{\prime} 0_{m-3}$, which has disutility only 1 for agents $4,5,6$. This implies that $f$ is not weakly group strategyproof.

Finally, for $n=4$, let now $f$ be the component-wise majority rule with $t=0_{m}$ (the case $t=1_{m}$ is analogous). Then $f\left(1100_{m-3}, 1010_{m-3}, 0110_{m-3}, 0_{m}\right)=0_{m}$, whereas $f\left(1110_{m-3}, 1110_{m-3}, 1110_{m-3}, 0_{m}\right)=1110_{m-3}$. Hence, coalition $\{1,2,3\}$ strongly manipulates. Hence also in this case $f$ is not weakly group strategy-proof. 

domains

Nevertheless, the conditions in Proposition 4.17 do not exclude the existence of weakly group strategy-proof rules, as the following example shows.

Example 4.18. Let $m=3$ and $n \geq 2$. We define a weakly group strategy-proof rule.

Say an alternative (or an agent) is of type 0 if it (or its preference) is in the set $\{000,001,010,100\}$ and type 1 if it (or its preference) is in the set $\{011,101,110,111\}$. A preference profile is of type 0 if it contains at least $\frac{n}{2}$ preferences of type 0 , and of type 1 otherwise. We define the rule $f$ as follows. Consider a profile $a^{N}$ of type 0 . If there is a component $j \in\{1,2,3\}$ such that $a_{j}^{i}=1$ for each $i \in N$, then $f\left(a^{N}\right)=a$ where $a_{j}=1$ and $a_{k}=0$ for $k \neq j$. If there is no such $j$, then $f\left(a^{N}\right)=$ 000. Now consider a profile $a^{N}$ of type 1. If there is a component $j \in\{1,2,3\}$ such that $a_{j}^{i}=0$ for each $i \in N$, then $f\left(a^{N}\right)=a$ where $a_{j}=0$ and $a_{k}=1$ for $k \neq j$. If there is no such $j$, then $f\left(a^{N}\right)=111$.

We show that this rule is weakly group strategy-proof. Assume the contrary, i.e., there exists a profile $a^{N}$, a coalition $S$ and a profile $b^{N}$ where $b^{i}=a^{i}$ for each agent $i \notin S$, such that each $i \in S$ strictly prefers $f\left(b^{N}\right)$ over $f\left(a^{N}\right)$, i.e., $r\left(f\left(b^{N}\right), a^{i}\right)<r\left(f\left(a^{N}\right), b^{i}\right)$ for all $i \in S$.

First consider the case where $f\left(a^{N}\right)=001$. Then, $a^{N}$ is of type 0 and for each $i \in N, a_{3}^{i}=1$. So every agent $i$ of type 0 has the preference 001 and hence $i \notin S$. This implies that at the new profile $b^{N}$ at least $\frac{n}{2}$ agents have preference 001 . Hence also $b^{N}$ is of type 0 and $f\left(b^{N}\right)=000$ or $f\left(b^{N}\right)=001$, thus $f\left(b^{N}\right)=000$. Since for each $i \in N, a_{3}^{i}=1$, they all prefer 001 over 000 . This is a contradiction. The cases where $f\left(a^{N}\right) \in\{010,100,011,101,110\}$ are analogous.

Now consider the case where $f\left(a^{N}\right)=000$. Then, $a^{N}$ is of type 0 and for each $j \in\{1,2,3\}$ there is $i \in N$ such that $a_{j}^{i}=0$. First assume that $f\left(b^{N}\right)=001$. Fix an $i \in N$ satisfying $a_{3}^{i}=0$. As $f\left(b^{N}\right)=001$, we must have $b_{3}^{i}=1$, and hence $i \in S$. This is a contradiction since $i$ strictly prefers $000=f\left(a^{N}\right)$ over $001=f\left(b^{N}\right)$. Thus, $f\left(b^{N}\right) \neq 001$. 
Similarly one shows that $f\left(b^{N}\right) \neq 010$ and $f\left(B^{N}\right) \neq 100$. Since the disutility of any agent of type 0 at $f\left(a^{N}\right)=000$ is either 0 or 1 , and $f\left(b^{N}\right) \neq 010$ and $f\left(B^{N}\right) \neq 100$, it follows that none of the agents of type 0 is in $S$, so profile $b^{N}$ is also of type 0 . Hence, $f\left(b^{N}\right)=000$, but this is a contradiction. An analogous argument works for the case where $f\left(a^{N}\right)=111$.

\subsection{Strong group strategy-proofness}

In this section we consider strongly group strategy-proof rules. We will show that if there are at least four agents and at least three alternatives then such rules do not exist. First, we consider the other cases and show that then strongly group strategy-proof rules exist.

Proposition 4.19. Let $n \in\{2,3\}$ and $m \geq 2$. Then each component-wise majority rule is strongly group strategy-proof.

Proof. If $n=2$, then this follows from Propositions 4.9 and 4.11. For the case $n=3$, again by Propositions 4.9 and 4.11, we only need to consider two-agent coalitions, and by the same argument as used in the proof of Proposition 4.15 it follows that no such coalition can deviate such that both members are at least as well off, with one strictly better off. This concludes the proof.

For $m=2$ and $n \geq 4$ define the rule $g$ as follows. If $a^{N}$ is a preference profile such that $a^{i}=00$ for some $i \in N$, then $g\left(a^{N}\right)=00$ (hence 00 is a dominant alternative). Otherwise, let $g$ be component-wise majority with tie-breaker 00 .

Proposition 4.20. $g$ is strongly group strategy-proof. 

domains

Proof. Let $a^{N}$ be a preference profile. If $a^{i}=00$ for some $i \in N$, then no coalition can weakly manipulate $g$ at $a^{N}$.

Otherwise, $a^{i} \neq 00$ for all $i \in N$. (i) If $g\left(a^{N}\right)=00$ then $n$ is even and $a^{N}=\left(10^{\left[\frac{n}{2}\right]}, 01^{\left[\frac{n}{2}\right]}\right)$, and it is easy to see that $g$ cannot be weakly manipulated at this profile. (ii) If $g\left(a^{N}\right)=11$ then changing to 00 is weakly or strictly worse for every agent; changing to 10 is strictly better for all agents with preference 10 but strictly worse for all other agents, and thus no coalition can weakly manipulate; similarly for changing to 01 . (iii) If $g\left(a^{N}\right)=10$, then any coalition of agents with preference 01 or 11 can only achieve 00 (other than 10), but 00 is strictly worse for agents with preference 11 and cannot be achieved by agents with preference 01 alone. (iv) The case $g\left(a^{N}\right)=01$ is analogous to (iii).

Summarizing, we have established that strongly group strategy-proof rules exist if $n=2$ and if $n=3$, and if $m=2$. It turns out that this no longer holds in all other cases. To show this, we will use Proposition 4.22 below. In order to prove this result, we need the following lemma, which in fact holds for weakly group strategy-proof rules. Note that by Lemma 4.7, every weakly group strategy-proof rule has a switching point.

Lemma 4.21. Let $f$ be a weakly group strategy-proof rule. Let $n_{0}$ be the switching point of $f$. Then:

[1] if $l<n_{0}$, then $f\left(0_{m}^{[l]},\left(1_{k} 0_{m-k}\right)^{[n-l]}\right)=1_{k} 0_{m-k}$ for all $k \geq 2$;

[2] if $l \geq n_{0}$, then $f\left(1_{m}^{[n-l]},\left(0_{k} 1_{m-k}\right)^{[l]}\right)=0_{k} 1_{m-k}$ for all $k \geq 2$.

Proof. We only prove [1], the proof of [2] is analogous. Let $l<n_{0}$.

For $m=2$, the statement reduces to $f\left(0_{2}^{l}, 1_{2}^{[n-l]}\right)=1_{2}$, which is true since $l<n_{0}$. Now assume that $m \geq 3$. Denote by $V^{k}$ the profile $\left(0_{m}^{l},\left(1_{k} 0_{m-k}\right)^{[n-l]}\right)$. We prove that $f\left(V^{k}\right)=1_{k} 0_{m-k}$ for all $k=2, \ldots, m$, by using backward induction on $k$. 
For $k=m$, the statement follows from the fact that $l<n_{0}$. Assume it to be true for $k+1$ where $k \geq 2$. We prove that it is also true for $k$. Since $V^{k}$ is symmetric in each pair of components $j, j^{\prime} \in\{1, \ldots, k\}$, Lemma 4.5 implies $f\left(V^{k}\right)_{j}=f\left(V^{k}\right)_{j^{\prime}}$ for all such $j$ and $j^{\prime}$. Since $f$ is weakly group strategy-proof, by Lemma 4.14 it is also weakly Pareto optimal and therefore component-wise unanimous. Hence, $f\left(V^{k}\right)_{j}=0$ for each $j \in\{k+1, \ldots, m\}$. Thus, either $f\left(V^{k}\right)=1_{k} 0_{m-k}$ or $f\left(V^{k}\right)=0_{m}$.

If $f\left(V^{k}\right)=0_{m}$, then the $n-l$ agents with preference $1_{k} 0_{m-k}$ can report $1_{k+1} 0_{m-(k+1)}$ and achieve $1_{k+1} 0_{m-(k+1)}$ by the induction hypothesis. The disutility of $1_{k+1} 0_{m-(k+1)}$ is equal to 1 for each of these agents, which is strictly better than the disutility $k$ of $0_{m}$. This contradicts weak group strategy-proofness of $f$. Therefore, $f\left(V^{k}\right)=1_{k} 0_{m-k}$, which concludes the proof of the induction step and of the lemma.

Proposition 4.22. Let $n \geq 4$ and $m \geq 3$, and let $f$ be a strongly group strategy-proof rule. Then $f$ has a dominant alternative.

Proof. Suppose, to the contrary, that $f$ has no dominant alternative. Then by Lemma $4.7 f$ has a switching point $n_{0}$ with $2 \leq n_{0} \leq n-1$. For $k=1, \ldots, n-1$ denote by $V^{k}$ the profile $\left(0_{m}^{[k]}, 1_{m}^{[n-k]}\right)$. We have $f\left(V^{1}\right)=1_{m}$ and $f\left(V^{n-1}\right)=0_{m}$.

Case 1: $n$ is even.

Consider the profile $V=\left(0_{m}, 010_{m-2}^{\left[\frac{n}{2}-1\right]}, 100_{m-2}^{\left[\frac{n}{2}-1\right]}, 1_{m}\right)$. Since $V$ is symmetric in components 1 and 2, Lemma 4.5 implies $f(V)_{1}=f(V)_{2}$. By a similar argument, $f(V)_{j}=f(V)_{j^{\prime}}$ for all $j, j^{\prime} \in\{3, \ldots, m\}$. Thus, $f(V)$ is equal to $0_{m}, 0_{2} 1_{m-2}, 1_{2} 0_{m-2}$, or $1_{m}$.

If $f(V)=0_{2} 1_{m-2}$ or $f(V)=1_{m}$, agents $2, \ldots, n-1$ in the profile $V$ have disutility $m-1$. Since $f\left(0_{m}, 0_{m}^{[n-2]}, 1_{m}\right)=f\left(V^{n-1}\right)=0_{m}$, which alternative has disutility 1 for agents $2, \ldots, n-1$, coalition $\{2, \ldots, n-$ 1 ) can weakly (even strongly) manipulate at $V$, contradicting strong (even weak) group strategy-proofness of $f$. 

domains

Next, suppose that $f(V)=1_{2} 0_{m-2}$. Since $f\left(V^{n-1}\right)=0_{m}$, coalition $\{1, \ldots, n-1\}$ can weakly manipulate at $V$ by each member reporting $0_{m}$ : alternative $0_{m}$ is strictly preferred by agent 1 , and agents $2, \ldots, n-1$ are indifferent. Again, this contradicts the assumption that $f$ is strongly group strategy-proof.

Finally, consider the case $f(V)=0_{m}$. Since $F\left(0_{m}, 1_{2} 0_{m-2}\right)=1_{2} 0_{m-2}$ by Lemma 4.21 , coalition $\{2, \ldots, n\}$ can weakly manipulate at $V$ : $1_{2} 0_{m-2}$ is strictly preferred by agent $n$ and agents $2, \ldots, n-1$ are indifferent. This again contradicts the assumption that $f$ is strongly group strategy-proof, and completes the proof for the case that $n$ is even.

Case 2: $n$ is odd.

Now consider the profile $V=\left(00_{m}^{[2]}, 010_{m-2}^{\left[\frac{n-3}{2}\right]}, 100_{m-2}^{\left[\frac{n-3}{2}\right]}, 1_{m}\right)$. As before, by Lemma 4.5, $f(V)_{1}=f(V)_{2}$ and $f(V)_{j}=f(V)_{j^{\prime}}$ for all $j, j^{\prime} \in$ $\{3, \ldots, m\}$. Thus, $f(V)$ is equal to $0_{m}, 0_{2} 1_{m-2}, 1_{2} 0_{m-2}$, or $1_{m}$.

If $f(V)=0_{2} 1_{m-2}$ or $F(V)=1_{m}$, the disutility for agents $1, \ldots, n-1$ in the profile $V$ from $f(V)$ is at least $m-2$. Since $f\left(V^{n-1}\right)=0_{m}$, which has disutility at most 1 for agents $1, \ldots, n-1$, coalition $\{1, \ldots, n-1\}$ can weakly (even strongly) manipulate at $V$, contradicting strong (even weak) group strategy-proofness of $f$. (This argument is similar to the one in the analogous case for $n$ even.)

Next, suppose that $f(V)=1_{2} 0_{m-2}$. Since $f\left(V^{n-1}\right)=0_{m}$, which has disutility at most 1 for agents $1, \ldots, n-1$, coalition $\{1, \ldots, n-1\}$ can weakly manipulate at $V$, contradicting strong group strategy-proofness of $f$.

Finally, suppose $f(V)=0_{m}$. Consider the coalition $\{3, \ldots, n\}$. If every member of this coalition reports $1_{2} 0_{m-2}$, the new profile is $\left(00_{m}^{[2]}, 1_{2} 0_{m-2}^{[n-2]}\right)$. If $n_{0}>2$, then by Lemma 4.21, $f\left(0_{m}^{[2]}, 1_{2} 0_{m-2}^{[n-2]}\right)=1_{2} 0_{m-2}$, implying that $\{3, \ldots, n\}$ can weakly manipulate at $V$ (in particular, agent $n$ is strictly better off). Since $f$ is strongly group strategy-proof, this implies $n_{0}=2$. Now consider the 
profile $V^{\prime}=\left(0_{m}, 011_{m-2}^{\left[\frac{n-3}{2}\right]}, 101_{m-2}^{\left[\frac{n-3}{2}\right]}, 1_{m}^{[2]}\right)$. By a similar same argument as for profile $V$, we obtain $n_{0}=n-1$, contradicting the assumption that $n>3$. This completes the proof.

Remark 4.23. By slightly modifying its proof, it can be shown that Proposition 4.22 still holds for $n \geq 4$ and $m=2$.

Remark 4.24. Proposition 4.22 does not hold for $n=3$ and $m=3$. By enumerating all profiles and eliminating the outcomes which contradict strong strategy-proofness of a rule, it can be shown that the component-wise majority rule is the unique strong strategy-proof rule which does not have a dominant alternative. The straightforward, but long and tedious proof can be found in the Appendix.

Theorem 4.25. Let $n \geq 4$ and $m \geq 3$. Then there does not exist strongly $a$ group strategy-proof rule.

Proof. We assume that $f$ is a strongly group strategy-proof rule, and derive a contradiction. By Proposition 4.22, $f$ has a dominant alternative. By Lemma 4.6, without loss of generality, this dominant alternative is $0_{m}$.

Consider the profile $V=\left(100_{m-2}, 011_{m-2}, 110_{m-2}^{[n-2]}\right)$. Since $V$ is symmetric in any two components $j, j^{\prime} \in\{3, \ldots, m\}$, Lemma 4.5 implies $f(V)_{j}=f(V)_{j^{\prime}}$ for any such $j$ and $j^{\prime}$. Therefore, $f(V)$ is equal to one of the eight alternatives $x y z_{m-2}$ with $x, y, z \in\{0,1\}$.

First we show that $z=0$. If $z=1$, then $f(V)$ has disutility at least $m-2$ for agent 1 . If that agent reports $0_{m}$, then $f$ assigns the the dominant alternative $0_{m}$, resulting in disutility 1 for agent 1 , which is strictly better since $m \geq 4$. This contradicts the assumption that $f$ is strategy-proof. Hence $z=0$.

If $f(V)=010_{m-2}$, then agent 1 can report $0_{m}$ and is again better off. If $f(V)=100_{m-2}$, then agent 2 can report $0_{m}$ and is better off. Now assume that $f(V)=0_{m}$. If all $n$ agents report $110_{m-2}$, then $f$ assigns 
$110_{m-2}$ which is (at least) weakly preferred by every agent and strictly preferred by the agents $3, \ldots, n$. Hence, $f(V)=110_{m-2}$.

Consider the profile $V^{\prime}=\left(10_{m-1}, 01_{m-1}, 1_{m}^{[n-2]}\right)$. Since $V^{\prime}$ is symmetric in any two components $j, j^{\prime} \in\{2, \ldots, m\}$, Lemma 4.5 implies that $f(V)_{j}=f(V)_{j^{\prime}}$ for any such $j$ and $j^{\prime}$. Therefore, $f\left(V^{\prime}\right)$ is equal to one of the four alternatives $x y_{m-1}$ with $x, y \in\{0,1\}$.

If $y=1$, then the disutility of $f(V)$ for agent 1 is at least $m-1$. If agent 1 reports the dominant alternative $0_{m}$, then $f$ assigns $0_{m}$, which agent 1 strictly prefers. Thus, $y=0$. If $f\left(V^{\prime}\right)=100_{m-2}$, then agent 2 can report $0_{m}$ and be strictly better off. Hence, $f\left(V^{\prime}\right)=0_{m}$.

The disutility for agents $3, \ldots, n$ is $m$. If each of them reports $110_{m-2}$, the resulting profile is $V$ and $f(V)=110_{m-2}$. Agents $3, \ldots, n$ strictly prefer $110_{m-2}$ over $0_{m}$. This contradicts the assumption that $f$ is strongly group strategy-proof and completes the proof of the theorem.

\subsection{Concluding remarks}

We have studied social choice functions on a multidimensional binary domain with preferences determined by Hamming distance. Under the natural conditions of unanimity, anonymity and neutrality, we have focused on strategy-proofness conditions: individual, weak group, and strong group strategy-proofness, with results ranging from many possibilities to impossibility.

From our analysis, in particular of special cases, it appears that it will be a difficult if not infeasible task to find all strategy-proof or weakly group strategy-proof rules, unless additional requirements are imposed. 


\section{Appendix}

We show that for $m=3$ and $n=3$, the component-wise majority rule is the unique strong strategy-proof rule which does not have a dominant alternative. We start by considering any strong strategy-proof rule $f$ which does not have a dominant alternative. Hence, $f$ is also component-wise unanimous. The idea is to enumerate all the preference profiles and step by step eliminate the outcomes for each profile, which contradict strong strategy-proofness of $f$. In the end, we show that $f$ is the component-wise majority rule.

As $f$ is unanimous, anonymous and neutral, we only need to consider the following profiles. Once the outcomes for these profiles are fixed, outcomes of all the other profiles are also determined by using unanimity, anonymity and neutrality of $f$.

\begin{tabular}{|c|c|c|c|}
\hline $000,000,001$ & $000,001,001$ & $000,000,100$ & $001,001,100$ \\
\hline $001,010,100$ & $000,111,111$ & $000,011,111$ & $000,011,011$ \\
\hline $000,011,110$ & $001,111,111$ & $001,011,111$ & $001,110,111$ \\
\hline $001,011,011$ & $001,110,110$ & $001,011,110$ & $001,101,011$ \\
\hline \hline $111,111,110$ & $111,110,110$ & $111,111,011$ & $110,110,011$ \\
\hline $110,101,011$ & $111,000,000$ & $111,100,000$ & $111,100,100$ \\
\hline $111,100,001$ & $110,000,000$ & $110,100,000$ & $110,001,000$ \\
\hline $110,100,100$ & $110,001,001$ & $110,100,001$ & $110,010,100$ \\
\hline
\end{tabular}

For every profile, there are 8 possible outcomes from the set $\{000,001,010,100,011,101,110,111\}$. We start by eliminating the outcomes, which contradict either unanimity, anonymity, neutrality or the fact that $f$ is strong group strategy-proof.

(1) For $f(001,010,100)$, we can eliminate all the outcomes except 000 and 11 because the other outcomes violate neutrality or anonymity. Moreover, we can also eliminate 111, because since all the agents strictly prefer 000 , over 111 , they can all misreport to 00 and obtain 000. Hence, $f(\mathbf{0 0 1}, \mathbf{0 1 0}, \mathbf{1 0 0})=\mathbf{0 0 0}$. 

domains

Analogously, $f(110,101,011)=111$.

(2) For $f(000,000,001)$, we can eliminate all the outcomes except 000 because otherwise the two agents with top alternatives 000 can misreport to 010 and 100 respectively and get the outcome 000 (refer step 1 and anonymity $)$. Hence, $f(000,000,001)=000$.

Analogously, $f(111,111,110)=111$.

Analogously for $f(111,111,110)$, we can eliminate all the outcomes except 111 and 110 .

(3) For $f(000,001,001)$, we can eliminate all the outcomes except 000 and 001 because the other outcomes violate component-wise unanimity at components either 1 or 2 .

Analogously for $f(111,110,110)$, we can eliminate all the outcomes except 111 and 110 .

(4) For $f(000,001,100)$, we can eliminate all the outcomes except 000, because otherwise 000 can misreport to 010 to obtain the outcome 000 (refer step 1 and anonymity). Hence, $f(\mathbf{0 0 0}, \mathbf{0 0 1}, \mathbf{1 0 0})=\mathbf{0 0 0}$.

Analogously, $f(111,110,011)=111$.

(5) For $f(001,001,100)$, we can eliminate the outcomes 010, 011, 110 and 111 because the they violate component-wise unanimity at component 2. We can also eliminate the outcome 100, because otherwise an agent with top alternative 001 can misreport to 010 and strictly preferred outcome 000 (refer step 1 and anonymity).

Analogously, for $f(110,110,011)$, we can eliminate the outcomes 101, 100, 001,000 and 011.

(6) For $f(000,011,111)$, we can eliminate all the outcomes except 000 and 111 because the they violate neutrality or anonymity. Moreover, we can eliminate 000, otherwise $f$ has a dominant alternative 000 . Hence, $f(000,111,111)=111$.

Analogously, $f(000,010,100)=000$. 
(7) For $f(000,011,111)$, we can eliminate the outcomes 000, 101, 110 and 100 because otherwise agent with top alternative 011 can misreport to 111 to obtain the strictly preferred outcome 111 (refer step 6).

Analogously, for $f(111,100,000)$, we can eliminate the outcomes 111, 010, 001 and 011.

(8) For $f(000,011,011)$, we can eliminate the outcomes 001, 010, 101, and 110 because they violate neutrality or anonymity. We can also eliminate 000 and 100, because otherwise the two agents with top alternative 011 can misreport to 111 to obtain strictly preferred outcome 111 (refer step 6). Finally the outcome 111 is also eliminated, since it violates the component-wise unanimity at component 1 . Hence, $f(000,011,011)=011$.

Analogously, $f(111,100,100)=100$.

(9) For $f(000,011,110)$, we can eliminate the outcomes 001, 100, 011, and 110 because they violate neutrality or anonymity. We can also eliminate 000 and 101, because agents with top alternatives 011 and 110 both can misreport to obtain a strictly better outcome 111. Finally, assume $f(000,011,110)=111$. If all three agents misreport to 010, agents 2 and 3 are indifferent, and agent 1 is strictly better. As $f$ is strong group strategy-proof, this is a contradiction, so 111 can be eliminated. Hence, $f(\mathbf{0 0 0}, \mathbf{0 1 1}, \mathbf{1 1 0})=\mathbf{0 1 0}$.

Analogously, $f(111,100,001)=101$.

(10) For $f(001,111,111)$, we can eliminate the outcomes 010, 011, 100 , and 101 because they violate neutrality or anonymity. We can also eliminate 000 and 110, they violate the component-wise unanimity at component 3 . Assume that $f(001,111,111)=001$. Then in the profile $(000,111,111)$, the agent with top alternative 000 is strictly better off by misreporting 001, as he strictly prefers 001 over $111=f(000,111,111)$, hence contradiction. So, the outcome 001 is eliminated. Hence, $f(\mathbf{0 0 1}, \mathbf{1 1 1}, \mathbf{1 1 1})=\mathbf{1 1 1}$.

Analogously, $f(\mathbf{1 1 0}, 000,000)=000$. 
(11) For $f(001,011,111)$, we can eliminate the outcomes 000, 010, 100 and 110 because the they violate component-wise unanimity at component 3 . We can also eliminate 101, because otherwise the agent with top alternative 011 can misreport to 111 to obtain strictly preferred outcome 111 (refer step 10). Analogously, for $f(110,100,000)$, we can eliminate the outcomes 111, 101, 001, 011 and 010 .

(12) For $f(001,110,111)$, we can eliminate the outcomes 010, 100, 011 and 101 because they violate neutrality or anonymity. We can also eliminate the outcomes 000 and 001 because otherwise the agent with top alternative 110 can misreport to 111 to obtain strictly preferred outcome 111 (refer step 10). Finally, we can eliminate the outcome 110, because otherwise the agent wit top alternative 001 can misreport to 111 and strictly preferred outcome 111 (refer analogous statement of step 2 and anonymity). Hence, $\boldsymbol{f}(\mathbf{0 0 1}, \mathbf{1 1 0}, \mathbf{1 1 1})=\mathbf{1 1 1}$. Analogously, $f(110,001,000)=000$.

(13) For $f(001,011,011)$, we can eliminate all the outcomes except 001 and 011 because the other outcomes violate component-wise unanimity at components either 1 or 3 . Assume $f(001,011,011)=001$. Then in the profile $f(000,011,011)$, the agent with top alternative 000 has incentive to misreport to 001 to obtain strictly better outcome, a contradiction. So, we can also eliminate the outcome 001. Hence, $f(001,011,011)=011$. Analogously, $f(110,100,100)=100$.

(14) For $f(001,110,110)$, we can eliminate the outcomes 010, 100, 011 and 101 because they violate neutrality or anonymity. We can also eliminate the outcomes 000 and 001 because otherwise the agent with top alternative 110 can misreport to 111 to obtain strictly preferred outcome 111 (refer step 10). Analogously, for $f(110,001,001)$, we can eliminate the all the outcomes except 001 and 000.

(15) For $f(001,011,110)$, we can eliminate the outcomes 000 and 101 because otherwise the agents with top alternatives 011 and 110 can misreport to 111 to obtain strictly preferred outcome 111 (refer step 10). We can eliminate 110, because otherwise agent with top alternatives 001 can misreport to 101 to obtain strictly preferred outcome 
111 (refer step 1 and anonymity). We can eliminate 100, because otherwise agent with top alternatives 011 can misreport to 111 to obtain strictly preferred outcome 110 (refer step 12 and anonymity). We can also eliminate 001, because otherwise agent with top alternatives 110 can misreport to 111 to obtain strictly preferred outcome (refer step 11, $f(001,011,111)$ is not completely determined, but as we have eliminated 001, agent 110 strictly prefers this outcome). Analogously, for $f(110,100,001)$, we can eliminate the the outcomes 111, 010, 001, 011 and 110 .

(16) For $f(001,101,011)$, we can eliminate the outcomes 010, 100, 011 and 101 because they violate neutrality or anonymity. We can also eliminate the outcomes 000 and 110 because the other outcomes violate component-wise unanimity at components either 1 or 2 . Analogously, for $f(110,001,001)$, we can eliminate the all the outcomes except 001 and 000 .

By the above eliminations we are left with following possible outcomes for the given profiles. 
Chapter 4. Group strategy-proof rules in multidimensional binary domains

\begin{tabular}{|c|c||c|c|}
\hline $000,000,001$ & 000 & $111,111,110$ & 111 \\
\hline $000,001,001$ & 000,001 & $111,110,110$ & 111,110 \\
\hline $000,001,100$ & 000 & $111,110,011$ & 111 \\
\hline $001,001,100$ & $000,001,101$ & $110,110,011$ & $111,110,010$ \\
\hline $001,010,100$ & 000 & $110,101,011$ & 111 \\
\hline \hline $000,111,111$ & 111 & $111,000,000$ & 000 \\
\hline $000,011,111$ & $001,010,011,111$ & $111,100,000$ & $110,101,100,000$ \\
\hline $000,011,011$ & 011 & $111,100,100$ & 100 \\
\hline $000,011,110$ & 010 & $111,100,001$ & 101 \\
\hline \hline $001,111,111$ & 111 & $110,000,000$ & 000 \\
\hline $001,011,111$ & $001,011,111$ & $110,100,000$ & $110,100,000$ \\
\hline $001,110,111$ & 111 & $110,001,000$ & 000 \\
\hline $001,011,011$ & 011 & $110,100,100$ & 100 \\
\hline $001,110,110$ & 110,111 & $110,001,001$ & 001,000 \\
\hline $001,011,110$ & $010,011,111$ & $110,100,001$ & $101,100,000$ \\
\hline $001,101,011$ & 001,111 & $110,010,100$ & 110,000 \\
\hline
\end{tabular}

Further eliminations are as follows:

(17) Step 9 gives us $f(000,011,110)=010$. Using neutrality, we have $f(000,011,101)=001$. So for $f(000,001,001)$, we can eliminate 000 , because otherwise two agents with top alternatives 001 can misreport to 011 and 110 respectively to obtain 001 . Hence, $f(\mathbf{0 0 0}, \mathbf{0 0 1}, \mathbf{0 0 1})=$ 001.

Analogously, $f(111,110,110)=110$.

(18) For $f(000,011,111)$, we can eliminate the outcome 111 because otherwise agent with top alternatives 000 can misreport to 011 to obtain strictly preferred outcome 011 (refer step 17 and anonymity, neutrality). We can also eliminate the outcomes 001 and 010 because otherwise agent with top alternatives 111 can misreport to 011 to obtain strictly preferred outcome 011 (refer step 8). Hence, $f(000,011,111)=011$. 
Analogously, $f(111,100,000)=100$.

(19) For $f(001,011,111)$, we can eliminate the outcome 111 because otherwise agent with top alternatives 000 can misreport to 011 to obtain strictly preferred outcome 011 (refer step 17 and anonymity, neutrality). We can also eliminate the outcome 001, because otherwise agent with top alternatives 111 can misreport to 011 to obtain strictly preferred outcome 011 (refer step 8). Hence, $\boldsymbol{f}(\mathbf{0 0 1}, \mathbf{0 1 1}, \mathbf{1 1 1})=\mathbf{0 1 1}$.

Analogously, $f(110,100,000)=100$.

(20) Assume $f(001,110,110)=111$. Then in the profile $f(111,110,110)$, the agent with top alternative 111 has incentive to misreport to 001 to obtain strictly better outcome, a contradiction. So, for $f(001,110,110)$, we can eliminate the outcome 001. Hence, $f(001,110,110)=110$.

Analogously, $f(110,001,001)=001$.

(21) Assume $f(001,001,100)=000$. Then in the profile $(000,001,001)$ the agent with top alternative 000 has incentive to misreport to 100 and obtain strictly better outcome (refer step 17), a contradiction. So, we can eliminate 000 for $f(001,001,100)$. Now assume that $f(001,001,100)=101$, so by neutrality $f(010,010,100)=110$, and again by neutrality $f(100,100,010)=110$. Then in the profile $(110,100,100)$ the agent with top alternative 110 has incentive to misreport to 010 and obtain strictly better outcome (refer step 13 and anonymity), a contradiction. So, we can also eliminate 101 for $f(001,001,100)$. Hence, $\boldsymbol{f}(\mathbf{0 0 1}, \mathbf{0 0 1}, \mathbf{1 0 0})=\mathbf{0 0 1}$.

Analogously, $f(110,110,011)=110$.

(22) For $f(001,011,110)$, we can eliminate the outcomes 010 and 111 because otherwise agent with top alternative 001 can misreport to 011 to obtain strictly preferred outcome 011 (refer analogous statement of step 21 and anonymity, neutrality). Hence, $f(\mathbf{0 0 1}, \mathbf{0 1 1}, \mathbf{1 1 0})=\mathbf{0 1 1}$.

Analogously, $f(110,100,001)=100$. 
Chapter 4. Group strategy-proof rules in multidimensional binary domains

(23) For $f(001,101,011)$, we can eliminate the outcome 111 because otherwise agent with top alternative 001 can misreport to 101 to obtain strictly preferred outcome 101 (refer analogous statement of step 21 and anonymity, neutrality). Hence, $f(001,101,011)=011$.

Analogously, $f(110,010,100)=100$.

Hence, the complete enumeration of the profiles shows that $f$ is component-wise majority rule. This completes the proof. 


\section{Bibliography}

[1] Aditya Aradhye, János Flesch, Mathias Staudigl, and Dries Vermeulen. "Incentive compatibility in sender-receiver stopping games". In: arXiv preprint arXiv:2004.01910 (2020).

[2] Pak Hung Au and Keiichi Kawai. "Competitive disclosure of correlated information". In: Economic Theory (2019), pp. 1-33.

[3] Robert J Aumann and Sergiu Hart. "Long cheap talk". In: Econometrica 71.6 (2003), pp. 1619-1660.

[4] Amos Azaria, Zinovi Rabinovich, Sarit Kraus, Claudia V Goldman, and Ya'akov Gal. "Strategic advice provision in repeated human-agent interactions". In: Twenty-Sixth AAAI Conference on Artificial Intelligence. 2012.

[5] Marco Battaglini. "Multiple referrals and multidimensional cheap talk". In: Econometrica 70.4 (2002), pp. 1379-1401.

[6] Andreas Blume, Douglas V DeJong, Yong-Gwan Kim, and Geoffrey B Sprinkle. "Experimental evidence on the evolution of meaning of messages in sender-receiver games". In: The American Economic Review 88.5 (1998), pp. 1323-1340.

[7] Sirin Botan, Arianna Novaro, and Ulle Endriss. "Group manipulation in judgment aggregation". In: (2016).

[8] Steven Brams and Peter C Fishburn. Approval voting. Springer Science \& Business Media, 2007.

[9] Vincent P. Crawford and Joel Sobel. "Strategic Information Transmission". In: Econometrica 50.6 (1982), pp. 1431-1451.

[10] Franz Dietrich and Christian List. "Strategy-proof judgment aggregation". In: Economics \& Philosophy 23.3 (2007), pp. 269-300.

[11] Erik Ekström and Stephane Villeneuve. "On the value of optimal stopping games". In: Ann. Appl. Probab. 16.3 (Aug. 2006), pp. 1576-1596. DOI: $10.1214 / 105051606000000204$. 
[12] Jeffrey C Ely. “Beeps”. In: American Economic Review 107.1 (2017), pp. 31-53.

[13] Thomas S. Ferguson. "Who Solved the Secretary Problem?" In: Statist. Sci. 4.3 (Aug. 1989), pp. 282-289. DOI: 10 . 1214 / ss / 1177012493.

[14] Françoise Forges. "An approach to communication equilibria". In: Econometrica: Journal of the Econometric Society (1986), pp. 1375-1385.

[15] Matthew Gentzkow and Emir Kamenica. "Competition in persuasion". In: The Review of Economic Studies 84.1 (2016), pp. 300322.

[16] Allan Gibbard. "Manipulation of schemes that mix voting with chance". In: Econometrica: Journal of the Econometric Society (1977), pp. 665-681.

[17] Mikhail Golosov, Vasiliki Skreta, Aleh Tsyvinski, and Andrea Wilson. "Dynamic strategic information transmission". In: Journal of Economic Theory 151 (2014), pp. 304-341.

[18] Jerry R Green and Nancy L Stokey. "A two-person game of information transmission". In: Journal of Economic Theory 135.1 (2007), pp. 90-104.

[19] Takakazu Honryo. "Dynamic persuasion". In: Journal of Economic Theory 178 (2018), pp. 36-58.

[20] Simon Huttegger, Brian Skyrms, Pierre Tarrès, and Elliott Wagner. "Some dynamics of signaling games". In: Proceedings of the National Academy of Sciences 111.Supplement 3 (2014), pp. 1087310880.

[21] Emir Kamenica and Matthew Gentzkow. "Bayesian persuasion". In: American Economic Review 101.6 (2011), pp. 2590-2615.

[22] Frédéric Koessler, Marie Laclau, Jérôme Renault, and Tristan Tomala. “Long information design". In: (2019). 
[23] Frédéric Koessler, Marie Laclau, and Tristan Tomala. "Interactive information design". In: HEC Paris Research Paper No. ECO/SCD2018-1260 (2018).

[24] Vijay Krishna and John Morgan. "A model of expertise". In: The Quarterly Journal of Economics 116.2 (2001), pp. 747-775.

[25] Vijay Krishna and John Morgan. "The art of conversation: eliciting information from experts through multi-stage communication". In: Journal of Economic theory 117.2 (2004), pp. 147-179.

[26] Vijay Krishna and John Morgan. "The art of conversation: eliciting information from experts through multi-stage communication". In: Journal of Economic theory 117.2 (2004), pp. 147-179.

[27] Reshef Meir, Ariel D Procaccia, and Jeffrey S Rosenschein. "On the limits of dictatorial classification". In: Proceedings of the 9th International Conference on Autonomous Agents and Multiagent Systems: volume 1-Volume 1. 2010, pp. 609-616.

[28] Paul Milgrom and John Roberts. "Relying on the information of interested parties". In: The RAND Journal of Economics (1986), pp. 18-32.

[29] Roger B Myerson. "Multistage games with communication". In: Econometrica: Journal of the Econometric Society (1986), pp. 323358.

[30] Hans Peters, Souvik Roy, and Soumyarup Sadhukhan. Unanimous and strategy-proof probabilistic rules for single-peaked preference profiles on graphs. Tech. rep. Working Paper, 2019.

[31] Jérôme Renault, Eilon Solan, and Nicolas Vieille. "Dynamic sender-receiver games". In: Journal of Economic Theory 148.2 (2013), pp. 502-534.

[32] Jérôme Renault, Eilon Solan, and Nicolas Vieille. "Optimal dynamic information provision". In: Games and Economic Behavior 104 (2017), pp. 329-349. 
[33] Mark Allen Satterthwaite. "Strategy-proofness and Arrow's conditions: Existence and correspondence theorems for voting procedures and social welfare functions". In: Journal of economic theory 10.2 (1975), pp. 187-217.

[34] James Schummer and Rakesh V Vohra. "Strategy-proof location on a network". In: Journal of Economic Theory 104.2 (2002), pp. 405-428.

[35] Brian Skyrms. Signals: Evolution, learning, and information. Oxford University Press, 2010.

[36] Brian Skyrms. "The flow of information in signaling games". In: Philosophical Studies 147.1 (2010), p. 155.

[37] Eilon Solan and Nicolas Vieille. "Stopping games-recent results". In: Advances in Dynamic Games. Springer, 2005, pp. 235-245.

[38] Zoi Terzopoulou and Ulle Endriss. "Strategyproof judgment aggregation under partial information". In: Social Choice and Welfare 53.3 (2019), pp. 415-442. 


\section{Summary}

In the first part of the thesis, we study the sender-receiver games. In this part we study the behaviour of the partially informed receiver and fully informed sender. Receiver can use the information provided by the sender to make his decisions. However while making decisions based on this information, the receiver must take into consideration that the sender is strategic and self-interested.

In Chapter 2, we define the sender-receiver stopping games. This was the first model introduced which combines the features from dynamic sender-receiver games (where sender and receiver interact repeatedly for finitely or infinitely many periods) and the stopping games (where the duration of the game is determined by the actions of the players involved). In this setting the sender and the receiver interact repeatedly until the receiver stops the game. Payoff functions of the players are monotonically increasing in the state of the world. So both players prefer that the game ends when the state is high enough, although due to different payoff functions, they have different interpretation of the state being high enough.

Since the sender can only send one of the two possible messages to the sender, the receiver obtains very little information about the state of the world. If the state is high enough for the sender, he has incentive to send a message which is interpreted as the suggestion for the receiver to stop the game. And if the state is low, he has incentive to send the other message which is interpreted as the suggestion for the receiver to continue the game. Although the receiver may have different cardinal preferences, obeying the sender is a best response for the receiver.

In the finite horizon, the regular strategy profile, that is the strategy profile in which the sender sends the message according to his optimal threshold values (sincere strategy) and the receiver obeys the sender's message (obeying strategy) is a Perfect Bayesian Equilibrium (PBE), 
provided the payoffs are undiscounted or discounted with sufficiently large discount factor. Moreover, this is the unique responsive PBE, that is, $\mathrm{PBE}$ in which the receiver plays non-babbling strategy.

In the infinite horizon, uniqueness of responsive PBE also holds for discounted payoff functions with sufficiently large discount factor, among the essentially Markovian strategy profiles. However, when the payoffs are undiscounted, there is no responsive PBE. This is the consequence of the fact that, in the infinite horizon game when the payoffs are undiscounted, the sender always expects to obtain higher state in the future with probability 1 , so he always sends the suggestion to continue, and the receiver obeys. So the game never stops and the both players receive payoff zero.

In Chapter 3, we consider one of the natural extension of the senderreceiver games in which there are multiple senders. In the case of only one sender, under responsive PBE, the receiver must obey the sender's suggestions. In the setting with many senders, the receiver obtains more information about the state of the world, hence the receiver can make better informed decisions. Due to the complexity of the interaction between the senders and receiver, this setting is much more difficult to analyze. We only consider the finite horizon setting.

In this setting the obeying strategy is not relevant since the receiver have information about the state from multiple senders. The reactive strategy of the receiver is a strategy with threshold on the number messages, such that the receiver stops the game if he receives more number of 'quit' messages than the threshold and continues if he receives less number of 'quit' messages than the threshold. This strategy is the most natural extension of obeying strategy from the previous setting. The regular profile in this setting corresponds to the strategy profile in which each sender plays the sincere strategy and the receiver plays the reactive strategy. Regular strategy profile is a PBE. Moreover, no set of senders can collude together to obtain better payoff. However, the receiver may be able to collude with one or more senders to obtain 
strictly better payoff for all the players involved in the coalition. In this setting, the uniqueness of PBE does not hold.

We study the externality effect of an additional sender over the receiver and the remaining senders. With more senders, the receiver obtains more information about the state, however it does not necessarily mean that the receiver gets higher expected payoff. We construct specific example of games such that the receiver obtains strictly higher payoff in a game with one sender than another game with two senders, where one of the senders in two sender game has same payoff as in the first game. Having an additional sender may be strictly better, strictly worse or indifferent for the remaining senders when there are at least 2 remaining senders. But having an additional sender could only be strictly worse or indifferent for the remaining senders when he is the lone sender.

In the second part of thesis which consists Chapter 4, we consider a model of multidimensional binary domain. In this domain, agents who have a yes or no opinion on different issues called components, vote for alternatives which are binary vectors. The preferences of agents over the alternatives are determined by the number of disagreements on components with their top alternative. The objective of this chapter is to study if agents can manipulate the social choice functions by misreporting their true preferences.

We study rules, which are social choice functions that satisfy unanimity, anonymity and neutrality. It turns out the preference domain in this setting is highly restricted. So, the standard Gibbard-Satterthwaite theorem does not hold. Particularly, we show that the componentwise majority rules are strategy-proof. Strategy-proofness is a fairly weak concept in this setting, so the characterization of all strategyproof rules is not feasible. Due to this, we study stronger notion of the weak group strategy-proofness in which the group of agents can collectively try to manipulate the outcome of the social choice function which is strictly better for every agent in the coalition. Surprisingly, the component-wise majority rules are not weakly group strategy-proof. 
We show the existence of rules which are weakly group strategy-proof for certain cases.

Even stronger notion we study is strong group strategy-proofness, in which group of agents can collectively try to manipulate the outcome of the social choice function which is weakly better for every agent in the coalition and strictly better for at least one agent. We show that if there are at least 3 components and 4 agents, there does not exist any strong group strategy-proofness rule. In the case of 3 components and 3 agents, we show that component-wise majority is the unique strong group strategy-proof rule. 


\section{Impact of the Thesis}

In this thesis we study two lines of works, first the sender-receiver stopping games, which is part of dynamic game theory, and second the multidimensional binary domain which is part of voting theory in social choice theory. We mostly focus on theoretical aspects of these topics. In this kind of research we try to define settings that imitate the real world scenarios and to reason the behaviour of the players involved. On one hand, we want these settings to depict the real life scenario as accurately as possible. On the other hand, we want the settings to be simple enough so that we can find substantial results which will help us to explain the behaviour precisely. As the real world is very complicated, these two objectives often conflict with each other, so it is very important to find a good balance.

Sender-receiver games and many of the related settings model the interaction between two or more partially informed players, and study how the flow of information takes place. This setting corresponds to many scenarios, for instance, competing firms want to exchange the information about the demand for the product, the supply and features of the product from other firms, etc. Our setting of sender-receiver stopping games relates very closely to the scenario in which an investor (receiver) decides whether or not to sell the stocks owned, and takes recommendation from a financial expert (sender) who has their own motives, such as commission earned on the transaction.

In voting theory, the main objective is to design voting rules which satisfy desirable properties, such as strategy-proofness, non-dictatorship. Many of such rules are being used to determine the outcome of elections on different levels. Our model of multidimensional binary domains closely relates to the scenario where the Parliament must decide whether to approve or reject a number of bills. Each member has a preference over each bill, whether or not it should be approved. It is 
important in this scenario to have rules which can not be manipulated by any member individually or collectively. 


\section{About the author}

Aditya Aradhye was born on May 04, 1994 in Sangli, India. He completed high school in 2012 at Pune, India. He joined Chennai Mathematical Institute in Chennai for Bachelor in Mathematics and Computer Science. He obtained BSc degree in 2015 with specialization in Theoretical computer Science. Subsequently he obtained MSc in Computer Science from the same university.

In 2017, he joined Maastricht University as a PhD candidate under the supervision of Prof. Dr. Dries Vermeulen, Dr. Jànos Flesch and Dr. Mathias Staudigl. The outcomes of his research are presented in this thesis. In 2020, he joined Czech Technical University in Prague as a postdoctoral researcher. His research interests range across different topics in game theory, particularly repeated games with incomplete information, voting theory, algorithmic game theory and mechanism design. 